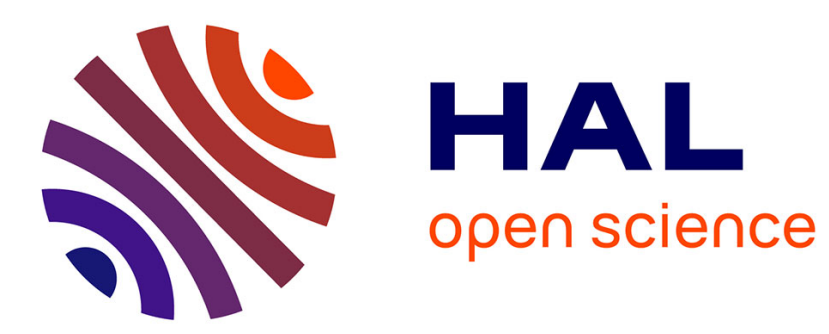

\title{
Small eigenvalues of the Neumann realization of the semiclassical Witten Laplacian
}

Dorian Le Peutrec

\section{To cite this version:}

Dorian Le Peutrec. Small eigenvalues of the Neumann realization of the semiclassical Witten Laplacian. Annales de la Faculté des Sciences de Toulouse. Mathématiques., 2010, 19 (3-4), pp.735-809. hal-00297207v3

\section{HAL Id: hal-00297207 \\ https://hal.science/hal-00297207v3}

Submitted on 23 Oct 2008

HAL is a multi-disciplinary open access archive for the deposit and dissemination of scientific research documents, whether they are published or not. The documents may come from teaching and research institutions in France or abroad, or from public or private research centers.
L'archive ouverte pluridisciplinaire HAL, est destinée au dépôt et à la diffusion de documents scientifiques de niveau recherche, publiés ou non, émanant des établissements d'enseignement et de recherche français ou étrangers, des laboratoires publics ou privés. 


\title{
Small eigenvalues of the Neumann realization of the semiclassical Witten Laplacian
}

\author{
D. Le Peutrec \\ IRMAR, UMR-CNRS 6625, Université de Rennes 1, \\ campus de Beaulieu, 35042 Rennes Cedex, France. \\ email: dorian.lepeutrec@univ-rennes1.fr
}

October 23, 2008

\begin{abstract}
This article follows the previous works [HKN] by Helffer-KleinNier and [HeNi1] by Helffer-Nier about the metastability in reversible diffusion processes via a Witten complex approach. Again, exponentially small eigenvalues of some self-adjoint realization of $\Delta_{f, h}^{(0)}=$ $-h^{2} \Delta+|\nabla f(x)|^{2}-h \Delta f(x)$, are considered as the small parameter $h>0$ goes to 0 . The function $f$ is assumed to be a Morse function on some bounded domain $\Omega$ with boundary $\partial \Omega$. Neumann type boundary conditions are considered. With these boundary conditions, some simplifications possible in the Dirichlet problem studied in [HeNi1] are no more possible. A finer treatment of the three geometries involved in the boundary problem (boundary, metric, Morse function) is carried out.
\end{abstract}

MSC 2000: 58J37 (58J10 58J32 60J60 81Q10 81Q20)

Keywords: Witten complex, Neumann boundary conditions, accurate asymptotics, exponentially small eigenvalues 


\section{Contents}

1 Introduction and result $\quad 3$

2 Witten Laplacian with Neumann boundary condition $\quad 7$

2.1 Introduction and notations . . . . . . . . . . . 7

2.2 Stokes and Green formulas . . . . . . . . . . . . . . . 9

2.3 Normal Neumann realization . . . . . . . . . . . . . . 11

3 First localization of the spectrum 15

3.1 Introduction and result . . . . . . . . . . . . . 15

3.2 A few preliminary lemmas . . . . . . . . . . . . 16

3.2.1 Variationnal results for the Witten Laplacian on $\mathbb{R}^{k}$. 16

3.2.2 The model half-space problem . . . . . . . . . . 18

3.2.3 Small eigenvalues for the model half-space problem . . 21

3.3 Proof of Theorem 3.1.5 . . . . . . . . . . . . . 28

4 Accurate WKB analysis near the boundary

for $\Delta_{f, h}^{(1)} \quad \mathbf{3 3}$

4.1 Introduction . . . . . . . . . . . . . . . 33

4.2 Local WKB construction . . . . . . . . . . . . . . . . 33

4.3 Another local Neumann realization of $\Delta_{f, h}^{(1)} \ldots \ldots \ldots . \ldots 35$

4.4 Exponential decay of eigenvectors of $\Delta_{f, h}^{N, D,(1)} \ldots \ldots \ldots 37$

4.5 Small eigenvalues are exponentially small . . . . . . . . . . 42

4.6 Accurate comparison with the WKB solution . . . . . . . 43

5 Labelling of local minima and construction of the quasimodes $\quad 49$

5.1 Preliminaries . . . . . . . . . . . . . . . . . . 49

5.2 Generalized critical points and local structure of the level sets of a Morse function . . . . . . . . . . . . . . . . 50

5.3 Labelling of local minima and first consequence . . . . . . . 52

5.4 Construction of the quasimodes . . . . . . . . . . . 54

5.5 Quasimodal estimates . . . . . . . . . . . . . 57

6 Final proof $\quad 64$

6.1 Main result . . . . . . . . . . . . . . . . 64

6.2 Finite dimensional reduction and final proof . . . . . . . . 64 


\section{Introduction and result}

In this text, we are interested in the exponentially small eigenvalues of the Neumann realization of the semiclassical Witten Laplacian $\Delta_{f, h}^{(0)}$ (acting on 0 -forms) on a connected compact Riemannian manifold with regular boundary.

Our purpose is to derive with the same accuracy as in [HKN] and in [HeNi1] asymptotic formulas for the smallest non zero eigenvalues of the Neumann realization of $\Delta_{f, h}^{(0)}$.

A similar problem was considered by many authors via a probabilistic approach in $[\mathrm{FrWe}],[\mathrm{HKS}],[\mathrm{Mic}]$, and $[\mathrm{Kol}]$. More recently, in the case of $\mathbb{R}^{n}$, accurate asymptotic forms of the exponentially small eigenvalues were obtained in [BEGK] and [BGK].

These results were improved and extended to the cases of boundaryless compact manifolds in $[\mathrm{HKN}]$ and of compact manifolds with boundaries for the Dirichlet realization of the Witten Laplacian in [HeNi1].

We want here to extend these last results to the case of compact manifolds with boundaries for the Neumann realization of the Witten Laplacian, that is with coherently deformed Neumann boundary conditions.

The function $f$ is assumed to be a Morse function on $\bar{\Omega}=\Omega \cup \partial \Omega$ with no critical points at the boundary. Furthermore, its restriction to the boundary $\left.f\right|_{\partial \Omega}$ is also assumed to be a Morse function.

From [ChLi], which completed results yet obtained in the boundaryless case (see [Sim2][Wit][CFKS][Hen][HeSj4][Hel3]), the number $m_{p}$ of eigenvalues of the Neumann realization of the Witten Laplacian $\Delta_{f, h}^{(p)}$ (acting on $p$-forms) in some interval $\left[0, C h^{\frac{3}{2}}\right]$ (for $h>0$ small enough) rely closely on the number of critical points of $f$ with index $p$.

In the boundaryless case, these numbers are exactly the numbers of critical points of $f$ with index $p$ in $\Omega$. Like in [HeNi1], they have to be increased in the case with boundary, taking into account the structure of the function $f$ at the boundary, $\left.f\right|_{\partial \Omega}$. Note furthermore that $m_{0}$ is here the number of local minima of $f$ in $\bar{\Omega}$.

Moreover, the first eigenvalue in our case is 0 and the other small eigenvalues are actually exponentially small as $h \rightarrow 0$, i.e. of order $e^{-\frac{C}{h}}$, where $C$ is a positive number independent of the small parameter $h>0$.

The point of view of $[\mathrm{HKN}]$ and [HeNi1] intensively uses, together with the techniques of [HeSj4], the two facts that the Witten Laplacian is associated with a cohomology complex and that the function $x \mapsto \exp -\frac{f(x)}{h}$ is a distributional solution in the kernel of the Witten Laplacian on 0-forms allowing to construct very efficiently quasimodes. 
Recall that the Witten Laplacian is defined as

$$
\Delta_{f, h}=d_{f, h} d_{f, h}^{*}+d_{f, h}^{*} d_{f, h},
$$

where $d_{f, h}$ is the distorted exterior differential

$$
d_{f, h}:=e^{-f(x) / h}(h d) e^{f(x) / h},
$$

and where $d_{f, h}^{*}$ is its adjoint for the $L^{2}$-scalar product canonically associated with the Riemannian structure (see for example [GHL][Gol][Sch]) The restriction of $d_{f, h}$ to $p$-forms is denoted by $d_{f, h}^{(p)}$. With these notations, the Witten Laplacian on functions is

$$
\Delta_{f, h}^{(0)}=d_{f, h}^{(0) *} d_{f, h}^{(0)} .
$$

In the Witten complex spirit and due to the relation

$$
d_{f, h}^{(0)} \Delta_{f, h}^{(0)}=\Delta_{f, h}^{(1)} d_{f, h}^{(0)},
$$

it is more convenient to consider the singular values of the restricted differential $d_{f, h}^{(0)}: F^{(0)} \rightarrow F^{(1)}$. The space $F^{(\ell)}$ is the $m_{\ell^{-}}$dimensional spectral subspace of $\Delta_{f, h}^{(\ell)}, \ell \in\{0,1\}$,

$$
F^{(\ell)}=\operatorname{Ran} 1_{I(h)}\left(\Delta_{f, h}^{(\ell)}\right),
$$

with $I(h)=\left[0, C h^{\frac{3}{2}}\right]$ and the property ${ }^{1}$

$$
1_{I(h)}\left(\Delta_{f, h}^{(1)}\right) d_{f, h}^{(0)}=d_{f, h}^{(0)} 1_{I(h)}\left(\Delta_{f, h}^{(0)}\right) .
$$

The restriction $\left.d_{f, h}\right|_{F^{(\ell)}}$ will be more shortly denoted by $\beta_{f, h}^{(\ell)}$

$$
\beta_{f, h}^{(\ell)}:=\left(d_{f, h}^{(\ell)}\right)_{/ F^{(\ell)}}
$$

We will mainly focus on the case $\ell=0$.

In order to exploit all the information which can be extracted from well chosen quasimodes, working with singular values of $\beta_{f, h}^{(0)}$ appears to be more efficient than considering their squares, the eigenvalues of $\Delta_{f, h}^{(0)}$. Those quantities agree better with the underlying Witten complex structure.

Note that in our case, 0 is the smallest eigenvalue of the (deformed) Neumann realization of the Witten Laplacian on 0 -forms due to the belonging of $x \mapsto \exp -\frac{f(x)}{h}$ to the domain of this operator (see Proposition 2.3.1 for

\footnotetext{
${ }^{1}$ The right end $a(h)=C h^{\frac{3}{2}}$ of the interval $I(h)=[0, a(h)]$ is suitable for technical reasons. What is important is that $a(h)=o(h)$. The value of $C>0$ does not play any role.
} 
the exact definition).

Let us now state the main result. Let $\mathcal{U}^{(0)}$ and $\mathcal{U}^{(1)}$ denote respectively the set of local minima and the set of generalized critical points with index 1 , or generalized saddle points, of the Morse function $f$ on $\bar{\Omega}$ (see Definition 5.2.1 for the exact meaning of "generalized"). The analysis requires an assumption which ensures that the exponentially small eigenvalues are simple with different logarithmic equivalent as $h \rightarrow 0$. Although it is possible to consider more general cases like in $[\mathrm{HKN}]$ and in [HeNi1], we will follow the point of view presented in $[\mathrm{Nie}]$ and work directly in a generic case which avoids some technical and unnecessary considerations.

Assumption 1.0.1. The critical values of $f$ and $\left.f\right|_{\partial \Omega}$ are all distinct and the quantities $f\left(U^{(1)}\right)-f\left(U^{(0)}\right)$, with $U^{(1)} \in \mathcal{U}^{(1)}$ and $U^{(0)} \in \mathcal{U}^{(0)}$ are distinct.

After this assumption, a one to one mapping $j$ from $\mathcal{U}^{(0)} \backslash\left\{U_{1}^{(0)}\right\}$ when $U_{1}^{(0)}$ is the global minimum, into the set $\mathcal{U}^{(1)}$ can be defined. The local minima are denoted by $U_{k}^{(0)}, k \in\left\{1, \ldots, m_{0}\right\}$, and the generalized saddle points by $U_{j}^{(1)}, j \in\left\{1, \ldots, m_{1}\right\}$. The ordering of the local minima as well as the one to one mapping $j$ will be specified in Subsection 5.3.

The final result will be expressed with the next quantities.

Definition 1.0.2. For $k \in\left\{2, \ldots, m_{0}\right\}$, we define:

$$
\begin{aligned}
& \gamma_{k}(h)= \begin{cases}\frac{\left|\operatorname{det} \operatorname{Hess} f\left(U_{k}^{(0)}\right)\right|^{\frac{1}{4}}}{(\pi h)^{\frac{n}{4}}} & \text { if } \quad U_{k}^{(0)} \in \Omega \\
\left(\frac{-2 \partial_{n} f\left(U_{k}^{(0)}\right)}{h}\right)^{\frac{1}{2}} \frac{\left.|\operatorname{det} \operatorname{Hess} f|_{\partial \Omega}\left(U_{k}^{(0)}\right)\right|^{\frac{1}{4}}}{(\pi h)^{\frac{n-1}{4}}} & \text { if } \quad U_{k}^{(0)} \in \partial \Omega,\end{cases} \\
& \delta_{j(k)}(h)=\left\{\begin{array}{lcc}
\frac{\left|\operatorname{det} \operatorname{Hess} f\left(U_{j(k)}^{(1)}\right)\right|^{\frac{1}{4}}}{(\pi h)^{\frac{n}{4}}} & \text { if } & U_{j(k)}^{(1)} \in \Omega \\
\left(\frac{-2 \partial_{n} f\left(U_{j(k)}^{(1)}\right)}{h}\right)^{\frac{1}{2}} \frac{\left.|\operatorname{det} \operatorname{Hess} f|_{\partial \Omega}\left(U_{j(k)}^{(1)}\right)\right|^{\frac{1}{4}}}{(\pi h)^{\frac{n-1}{4}}} & \text { if } & U_{j(k)}^{(1)} \in \partial \Omega,
\end{array}\right.
\end{aligned}
$$

and,

$$
\theta_{j(k)}(h)=\left\{\begin{array}{lcc}
\frac{h^{\frac{1}{2}}}{\pi^{\frac{1}{2}}} \frac{(\pi h)^{\frac{n}{2}}\left|\hat{\lambda}_{1}^{\Omega}\right|^{\frac{1}{2}}}{\mid \operatorname{det} \operatorname{Hess} f\left(\left.U_{j(k)}^{(1)}\right|^{\frac{1}{2}}\right.} & \text { if } & U_{j(k)}^{(1)} \in \Omega \\
\frac{h^{2}}{-2 \partial_{n} f\left(U_{j(k)}^{(1)}\right)} \frac{(\pi h)^{\frac{n-2}{2}}\left|\widehat{\lambda}_{1}^{\partial \Omega}\right|^{\frac{1}{2}}}{\left.|\operatorname{det} \operatorname{Hess} f| \partial \Omega\left(U_{j(k)}^{(1)}\right)\right|^{\frac{1}{2}}} & \text { if } & U_{j(k)}^{(1)} \in \partial \Omega,
\end{array}\right.
$$

where $\widehat{\lambda}_{1}^{W}$ is the negative eigenvalue of $\left.\operatorname{Hess} f\right|_{W}\left(U_{j(k)}^{(1)}\right)$ for $W=\Omega$ or $W=$ $\partial \Omega$. 


\section{Theorem 1.0.3.}

Under Assumption 1.0.1 and after the ordering specified in Subsection 5.3, there exists $h_{0}$ such that, for $h \in\left(0, h_{0}\right]$, the spectrum in $\left[0, h^{\frac{3}{2}}\right)$ of the Neumann realization of $\Delta_{f, h}^{(0)}$ in $\bar{\Omega}$ consists of $m_{0}$ eigenvalues $0=\lambda_{1}(h)<$ $\ldots<\lambda_{m_{0}}(h)$ of multiplicity 1 .

Moreover, the above $m_{0}-1$ non zero eigenvalues are exponentially small and admit the following asymptotic expansions:

$$
\lambda_{k}(h)=\gamma_{k}^{2}(h) \delta_{j(k)}^{2}(h) \theta_{j(k)}^{2}(h) e^{-2 \frac{f\left(U_{j(k)}^{(1)}\right)-f\left(U_{k}^{(0)}\right)}{h}}\left(1+h c_{k}^{1}(h)\right)
$$

where $\gamma_{k}(h), \delta_{j(k)}(h)$, and $\theta_{j(k)}(h)$ are defined in the above definition and $c_{k}^{1}(h)$ admits a complete expansion: $c_{k}^{1}(h) \sim \sum_{m=0}^{\infty} h^{m} c_{k, m}$.

This theorem extends to the case with Neumann boundary conditions the previous result of $[\mathrm{BGK}]$ and its improvements in [HKN] and [HeNi1] (see also non-rigorous formal computations of $[\mathrm{KoMa}]$, who look also at cases with symmetry and the books [FrWe] and [Kol] and references therein).

To prove this theorem, we will follow the same strategy as in [HKN] and in [HeNi1] and some intermediary results will be reused without demonstration (what will be indicated in the article). Moreover, some proofs will be improved (see for example the final proof reduced now to a simple Gaussian elimination explained in [Lep]).

At least, the geometry of the Neumann case is different from the geometry of the above references. This leads to different results (compare Theorem 1.0.3 and the main theorem of [HeNi1]) and some proofs have to be entirely reconsidered. In fact, the study of the Dirichlet realization of the Witten Laplacian done in [HeNi1] agreed better with the local geometry near the boundary, which led to simpler computations (see the local WKB construction in Section 4 for example).

The article is organized as follows.

In the second section, we analyze in detail the boundary complex adapted to our analysis in order to keep the commutation relation (1.0.4) (a part of the answer already existed in the literature (see [Sch], [Duf], [DuSp], [Gue], and $[\mathrm{ChLi}]$ ) in connection with the analysis of the relative or absolute cohomology as defined in [Gil]).

The third section is devoted to the proof of rough estimates (to get a precise localization of the spectrum of the Laplacian) replacing the harmonic oscillator approximation in the case without boundary.

These two sections bring no additional difficulties in comparison with what was done in [HeNi1].

In the fourth section, we give the WKB construction for an eigenform of the Witten Laplacian on 1-forms localized near a critical point of the boundary, according to the analysis done in [Lep1]. Moreover, it was possible in 
the Dirichlet case to use only a single coordinate system in order to approximate an eigenform by a WKB construction while different coordinate systems arise naturally here. Lemma 3.3 .1 will play a crucial role to juggle with these different coordinate systems.

In the first part of the fifth section we label the local minima and we construct the above injective map $j$ under Assumption 1.0.1.

In its second part, after having constructed adapted quasimodes to our analysis, we make some scalar estimates - using the Laplace method - which lead directly to the final proof of the theorem, using the result of [Lep], in the sixth section. Again, we cannot use like in [HeNi1] a single coordinate system and we must again call on Lemma 3.3.1 to be able to use the Laplace method. It is due to the local geometry near a generalized critical point with index 1 which is rather more complicated than in [HeNi1].

\section{Witten Laplacian with Neumann boundary con- dition}

\subsection{Introduction and notations}

This section is analogous to the second section of [HeNi1] and we will use the same notations that we recall here.

Let $\bar{\Omega}$ be a $\mathcal{C}^{\infty}$ connected compact oriented Riemannian $n$-dimensional manifold. We will denote by $g_{0}$ the given Riemannian metric on $\bar{\Omega} ; \Omega$ and $\partial \Omega$ will denote respectively its interior and its boundary.

The cotangent (resp. tangent) bundle on $\Omega$ is denoted by $T^{*} \Omega$ (resp. $T \Omega$ ) and the exterior fiber bundle by $\Lambda T^{*} \Omega=\oplus_{p=0}^{n} \Lambda^{p} T^{*} \Omega$ (resp. $\quad \Lambda T \Omega=$ $\oplus_{p=0}^{n} \Lambda^{p} T \Omega$.

The fiber bundles $\Lambda T \partial \Omega=\oplus_{p=0}^{n-1} \Lambda^{p} T \partial \Omega$ and $\Lambda T^{*} \partial \Omega=\oplus_{p=0}^{n-1} \Lambda^{p} T^{*} \partial \Omega$ are defined similarly.

The space of $\mathcal{C}^{\infty}, \mathcal{C}_{0}^{\infty}, L^{2}, H^{s}$, etc. sections in any of these fiber bundles, $E$, on $O=\Omega$ or $O=\partial \Omega$, will be denoted respectively by $\mathcal{C}^{\infty}(O ; E), \mathcal{C}_{0}^{\infty}(O ; E)$, $L^{2}(O ; E), H^{s}(O ; E)$, etc.

When no confusion is possible we will simply use the short notations $\Lambda^{p} \mathcal{C}^{\infty}$, $\Lambda^{p} \mathcal{C}_{0}^{\infty}, \Lambda^{p} L^{2}$ and $\Lambda^{p} H^{s}$ for $E=\Lambda^{p} T^{*} \Omega$ or $E=\Lambda^{p} T^{*} \partial \Omega$.

Note that the $L^{2}$ spaces are those associated with the unit volume form for the Riemannian structure on $\Omega$ or $\partial \Omega$ ( $\Omega$ and $\partial \Omega$ are oriented).

The notation $\mathcal{C}^{\infty}(\bar{\Omega} ; E)$ is used for the set of $\mathcal{C}^{\infty}$ sections up to the boundary. Finally since $\partial \Omega$ is $\mathcal{C}^{\infty}, \mathcal{C}^{\infty}(\bar{\Omega} ; E)$ is dense in $H^{s}(\Omega ; E)$ for $s \geq 0$ and the trace operator $\left.\omega \rightarrow \omega\right|_{\partial \Omega}$ extends to a surjective operator from $H^{s}(\Omega ; E)$ onto $H^{s-1 / 2}(\partial \Omega ; E)$ as soon as $s>1 / 2$. 
Let $d$ be the exterior differential on $\mathcal{C}_{0}^{\infty}\left(\Omega ; \Lambda T^{*} \Omega\right)$

$$
\left(d^{(p)}: \mathcal{C}_{0}^{\infty}\left(\Omega ; \Lambda^{p} T^{*} \Omega\right) \rightarrow \mathcal{C}_{0}^{\infty}\left(\Omega ; \Lambda^{p+1} T^{*} \Omega\right)\right)
$$

and $d^{*}$ its formal adjoint with respect to the $L^{2}$-scalar product inherited from the Riemannian structure

$$
\left(d^{(p), *}: \mathcal{C}_{0}^{\infty}\left(\Omega ; \Lambda^{p+1} T^{*} \Omega\right) \rightarrow \mathcal{C}_{0}^{\infty}\left(\Omega ; \Lambda^{p} T^{*} \Omega\right)\right) .
$$

Remark 2.1.1. Note that $d$ and $d^{*}$ are both well defined on $\mathcal{C}^{\infty}\left(\bar{\Omega} ; \Lambda T^{*} \Omega\right)$. We set, for a function $f \in \mathcal{C}^{\infty}(\bar{\Omega} ; \mathbb{R})$ and $h>0$, the distorted operators defined on $\mathcal{C}^{\infty}\left(\bar{\Omega} ; \Lambda T^{*} \Omega\right)$ :

$$
d_{f, h}=e^{-f(x) / h}(h d) e^{f(x) / h} \quad \text { and } \quad d_{f, h}^{*}=e^{f(x) / h}\left(h d^{*}\right) e^{-f(x) / h},
$$

The Witten Laplacian is the differential operator defined on $\mathcal{C}^{\infty}\left(\bar{\Omega} ; \Lambda T^{*} \Omega\right)$ by:

$$
\Delta_{f, h}=d_{f, h}^{*} d_{f, h}+d_{f, h} d_{f, h}^{*}=\left(d_{f, h}+d_{f, h}^{*}\right)^{2} .
$$

Remark 2.1.2. The last equality becomes from the property $d d=d^{*} d^{*}=0$ which implies:

$$
d_{f, h} d_{f, h}=d_{f, h}^{*} d_{f, h}^{*}=0 .
$$

It means, by restriction to the $p$-forms in $\mathcal{C}^{\infty}\left(\bar{\Omega} ; \Lambda^{p} T^{*} \Omega\right)$ :

$$
\Delta_{f, h}^{(p)}=d_{f, h}^{(p), *} d_{f, h}^{(p)}+d_{f, h}^{(p-1)} d_{f, h}^{(p-1), *} .
$$

Note that (2.1.2) imply that, for all $u$ in $\mathcal{C}^{\infty}\left(\bar{\Omega} ; \Lambda^{p} T^{*} \Omega\right)$,

$$
\begin{gathered}
\Delta_{f, h}^{(p+1)} d_{f, h}^{(p)} u=d_{f, h}^{(p)} \Delta_{f, h}^{(p)} u \\
\text { and } \\
\Delta_{f, h}^{(p-1)} d_{f, h}^{(p-1), *} u=d_{f, h}^{(p-1), *} \Delta_{f, h}^{(p)} u .
\end{gathered}
$$

We end up this section by a few relations with exterior and interior products (respectively denoted by $\wedge$ and $\mathbf{i}$ ), gradients (denoted by $\nabla$ ) and Lie derivatives (denoted by $\mathcal{L}$ ) which will be very useful:

$$
\begin{aligned}
(d f \wedge)^{*} & =\mathbf{i}_{\nabla f} \quad\left(\text { in } L^{2}\left(\bar{\Omega} ; \Lambda^{p} T^{*} \Omega\right)\right), \\
d_{f, h} & =h d+d f \wedge \\
d_{f, h}^{*} & =h d^{*}+\mathbf{i}_{\nabla f}, \\
d \circ \mathbf{i}_{X}+\mathbf{i}_{X} \circ d & =\mathcal{L}_{X}, \\
\Delta_{f, h} & =h^{2}\left(d+d^{*}\right)^{2}+|\nabla f|^{2}+h\left(\mathcal{L}_{\nabla f}+\mathcal{L}_{\nabla f}^{*}\right),
\end{aligned}
$$

where $X$ denotes a vector field on $\Omega$ or $\bar{\Omega}$.

Remark 2.1.3. We work here on a Riemannian manifold and the operators introduced depend on the Riemannian metric $g_{0}$. Nevertheless, we have omitted here this dependence for conciseness. 


\subsection{Stokes and Green formulas}

In order to define suitably the self-adjoint Neumann realization of the Witten Laplacian $\Delta_{f, h}$ that we will study in the rest of this work, we need variants from the Stokes and the Green formulas.

For that, we use some notations and properties which are very convenient for boundary problems and which are introduced for example in [Sch] and recalled in [HeNi1].

Definition 2.2.1. We denote by $\vec{n}_{\sigma}$ the outgoing normal at $\sigma \in \partial \Omega$ and by $\vec{n}_{\sigma}^{*}$ the 1-form dual to $\vec{n}_{\sigma}$ for the Riemannian scalar product.

For any $\omega \in \mathcal{C}^{\infty}\left(\bar{\Omega} ; \Lambda^{p} T^{*} \Omega\right)$, the form $\mathbf{t} \omega$ is the element of $\mathcal{C}^{\infty}\left(\partial \Omega ; \Lambda^{p} T^{*} \Omega\right)$ defined by:

$$
(\mathbf{t} \omega)_{\sigma}\left(X_{1}, \ldots, X_{p}\right)=\omega_{\sigma}\left(X_{1}^{T}, \ldots, X_{p}^{T}\right), \forall \sigma \in \partial \Omega,
$$

with the decomposition into the tangential and normal components to $\partial \Omega$ at $\sigma: X_{i}=X_{i}^{T} \oplus x_{i}^{\perp} \vec{n}_{\sigma}$.

Moreover,

$$
(\mathbf{t} \omega)_{\sigma}=\mathbf{i}_{\vec{n}_{\sigma}}\left(\vec{n}_{\sigma}^{*} \wedge \omega_{\sigma}\right) .
$$

The projected form $\mathbf{t} \omega$, which depends on the choice of $\vec{n}_{\sigma}$ (i.e. on $g_{0}$ ), can be compared with the canonical pull-back $j^{*} \omega$ associated with the imbedding $j: \partial \Omega \rightarrow \Omega$. Actually the exact relationship is $j^{*} \omega=j^{*}(\mathbf{t} \omega)$. With an abuse of notation, the form $j^{*}(\mathbf{t} \omega)$ will be simply written $\mathbf{t} \omega$ for example in Stokes formula without any possible confusion.

The normal part of $\omega$ on $\partial \Omega$ is defined by:

$$
\mathbf{n} \omega=\left.\omega\right|_{\partial \Omega}-\mathbf{t} \omega \quad \in \mathcal{C}^{\infty}\left(\partial \Omega ; \Lambda^{p} T^{*} \Omega\right) .
$$

If necessary $\mathbf{t} \omega$ and $\mathbf{n} \omega$ can be considered as elements of $\mathcal{C}^{\infty}\left(\bar{\Omega} ; \Lambda^{p} T^{*} \Omega\right)$ by a variant of the collar theorem (see [HeNi1] or [Sch] for details).

The Hodge operator $\star$ is locally defined in a pointwise orthonormal frame $\left(E_{1}, \ldots, E_{n}\right)$ by:

$$
\left(\star \omega_{x}\right)\left(E_{\sigma(p+1)}, \ldots, E_{\sigma(n)}\right)=\varepsilon(\sigma) \omega_{x}\left(E_{\sigma(1)}, \ldots, E_{\sigma(p)}\right),
$$

for $\omega_{x} \in \Lambda^{p} T_{x}^{*} \Omega$ and with any permutation $\sigma \in \Sigma(n)$ of $\{1, \ldots, n\}$ preserving $\{1, \ldots, p\}(\varepsilon(\sigma)$ denotes the signature of $\sigma)$.

We recall the formulas:

$$
\begin{array}{cc}
\star\left(\star \omega_{x}\right)=(-1)^{p(n-p)} \omega_{x}, & \forall \omega_{x} \in \Lambda^{p} T_{x}^{*} \Omega, \\
\left\langle\omega_{1} \mid \omega_{2}\right\rangle_{\Lambda^{p} L^{2}}=\int_{\Omega} \omega_{1} \wedge \star \overline{\omega_{2}}, & \forall \omega_{1}, \omega_{2} \in \Lambda^{p} L^{2},
\end{array}
$$


and:

$$
\begin{aligned}
\star d^{*,(p-1)}=(-1)^{p} d^{(n-p)} \star, & \star d^{(p)}=(-1)^{p+1} d^{*,(n-p-1)} \star, \\
\star \mathbf{n}=\mathbf{t} \star, & \star \mathbf{t}=\mathbf{n} \star, \\
\mathbf{t} d=d \mathbf{t}, & \mathbf{n} d^{*}=d^{*} \mathbf{n} .
\end{aligned}
$$

With the previous convention $j^{*}(\mathbf{t} \omega)=\mathbf{t} \omega$, the Stokes formula writes:

$$
\forall \omega \in \mathcal{C}^{\infty}\left(\bar{\Omega} ; \Lambda^{p} T^{*} \Omega\right), \quad \int_{\Omega} d \omega=\int_{\partial \Omega} j^{*} \omega=\int_{\partial \Omega} \mathbf{t} \omega,
$$

and a first deformed Green formula given in [HeNi1] states that

$$
\begin{aligned}
& \left\langle d_{f, h} \omega \mid d_{f, h} \eta\right\rangle_{\Lambda^{p+1} L^{2}}+\left\langle d_{f, h}^{*} \omega \mid d_{f, h}^{*} \eta\right\rangle_{\Lambda^{p-1} L^{2}} \\
= & \left\langle\Delta_{f, h} \omega \mid \eta\right\rangle_{\Lambda^{p} L^{2}}+h \int_{\partial \Omega}(\mathbf{t} \bar{\eta}) \wedge\left(\star \mathbf{n} d_{f, h} \omega\right)-h \int_{\partial \Omega}\left(\mathbf{t} d_{f, h}^{*} \omega\right) \wedge(\star \mathbf{n} \bar{\eta})
\end{aligned}
$$

holds for all $\omega \in \Lambda^{p} H^{2}$ and $\eta \in \Lambda^{p} H^{1}$. This formulation of (2.2.7) does not depend on the choice of an orientation. If $\mu$ and $\mu_{\partial \Omega}$ denote the volume forms in $\Omega$ and $\partial \Omega$, the orientation is chosen such that $\left(\mu_{\partial \Omega}\right)_{\sigma}\left(X_{1}, \ldots, X_{n-1}\right)=$ $\mu_{\sigma}\left(\vec{n}_{\sigma}, X_{1}, \ldots, X_{n-1}\right)$. A simple computation in normal frames (see [Sch], prop. 1.2.6) leads to:

$$
\mathbf{t} \omega_{1} \wedge \star \mathbf{n} \overline{\omega_{2}}=\left\langle\omega_{1} \mid \mathbf{i}_{\vec{n}_{\sigma}} \omega_{2}\right\rangle_{\Lambda^{p} T_{\sigma}^{*} \Omega} d \mu_{\partial \Omega},
$$

for $\omega_{1} \in \mathcal{C}^{\infty}\left(\bar{\Omega} ; \Lambda^{p} T^{*} \Omega\right)$ and $\omega_{2} \in C^{\infty}\left(\bar{\Omega} ; \Lambda^{p+1} T^{*} \Omega\right)$.

Definition 2.2.2. We denote by $\frac{\partial f}{\partial n}(\sigma)$ or $\partial_{n} f(\sigma)$ the normal derivative of $f$ at $\sigma$ :

$$
\frac{\partial f}{\partial n}(\sigma)=\partial_{n} f(\sigma):=\left\langle\nabla f(\sigma) \mid \vec{n}_{\sigma}\right\rangle .
$$

As a consequence of (2.2.8) we get the following useful decomposition formula.

Lemma 2.2.3. (Normal Green Formula)

The identity

$$
\begin{gathered}
\left\|d_{f, h} \omega\right\|_{\Lambda^{p+1} L^{2}}^{2}+\left\|d_{f, h}^{*} \omega\right\|_{\Lambda^{p-1} L^{2}}^{2} \\
+\||\nabla f| \omega\|_{\Lambda^{p} L^{2}}^{2}+h\left\langle\left(\mathcal{L}_{\nabla f}^{2}+\mathcal{L}_{\nabla f}^{*}\right) \omega\right| \omega \|_{\Lambda^{p} L^{2}}^{2} \\
+h \int_{\partial \Omega}\langle\omega \mid \omega\rangle_{\Lambda^{p} T_{\sigma}^{*} \Omega}\left(\frac{\partial f}{\partial n}\right)(\sigma) d \mu_{\partial \Omega}
\end{gathered}
$$

holds for any $\omega \in \Lambda^{p} H^{1}$ such that $\mathbf{n} \omega=0$. 
Proof.

Since $\mathcal{C}^{\infty}\left(\bar{\Omega} ; \Lambda^{p} T^{*} \Omega\right)$ is dense in $\Lambda^{p} H^{1}$, while both terms of the identity are continuous on $\Lambda^{p} H^{1}$, the form $\omega$ can be assumed to be in $\mathcal{C}^{\infty}\left(\bar{\Omega} ; \Lambda^{p} T^{*} \Omega\right)$.

We use the relation (2.2.7) with both $f=0\left(d_{0, h}=h d\right.$ and $\left.d_{0, h}^{*}=h d^{*}\right)$ and a general $f \in \mathcal{C}^{\infty}(\bar{\Omega} ; \mathbb{R})$. We obtain:

$$
\begin{gathered}
\left\|d_{f, h} \omega\right\|_{\Lambda^{p+1} L^{2}}^{2}+\left\|d_{f, h}^{*} \omega\right\|_{\Lambda^{p-1} L^{2}}^{2}-h^{2}\|d \omega\|_{\Lambda^{p+1} L^{2}}^{2}-h^{2}\left\|d^{*} \omega\right\|_{\Lambda^{p-1} L^{2}}^{2}= \\
\left\langle\left(\Delta_{f, h}-\Delta_{0, h}\right) \omega \mid \omega\right\rangle_{\Lambda^{p} L^{2}}+h \int_{\partial \Omega}(\mathbf{t} \bar{\omega}) \wedge \star \mathbf{n}(d f \wedge \omega)-h \int_{\partial \Omega}\left(\mathbf{t i}_{\nabla f} \omega\right) \wedge(\star \mathbf{n} \bar{\omega}) \\
=\left\langle\left(\Delta_{f, h}-\Delta_{0, h}\right) \omega \mid \omega\right\rangle_{\Lambda^{p} L^{2}}+h \int_{\partial \Omega}\left\langle\omega \mid \mathbf{i}_{\vec{n}_{\sigma}}(d f \wedge \omega)\right\rangle_{\Lambda T_{\sigma}^{*} \Omega} d \mu_{\partial \Omega} .
\end{gathered}
$$

By (2.1.9):

$$
\left\langle\left(\Delta_{f, h}-\Delta_{0, h}\right) \omega \mid \omega\right\rangle_{\Lambda^{p} L^{2}}=\||\nabla f| \omega\|_{\Lambda^{p} L^{2}}^{2}+h\left\langle\left(\mathcal{L}_{\nabla f}+\mathcal{L}_{\nabla f}^{*}\right) \omega \mid \omega\right\rangle_{\Lambda^{p} L^{2}} .
$$

For the integral term, we write:

$$
\begin{aligned}
\mathbf{i}_{\vec{n}_{\sigma}}(d f \wedge \omega)\left(X_{1}, \ldots, X_{p}\right) & =(d f \wedge \omega)\left(\vec{n}_{\sigma}, X_{1}, \ldots, X_{p}\right) \\
& =d f\left(\vec{n}_{\sigma}\right) \cdot \omega\left(X_{1}, \ldots, X_{p}\right) \quad \text { because } \mathbf{n} \omega=0 \\
& =\left\langle\nabla f(\sigma) \mid \vec{n}_{\sigma}\right\rangle \cdot \omega\left(X_{1}, \ldots, X_{p}\right) \\
& =\left(\frac{\partial f}{\partial n}(\sigma)\right) \omega\left(X_{1}, \ldots, X_{p}\right),
\end{aligned}
$$

which proves the lemma.

\subsection{Normal Neumann realization}

In this subsection, we specify the self-adjoint realization of $\Delta_{f, h}^{(0)}$ in which we are interested. Like in [HeNi1], we want this self-adjoint realization (denoted by $\Delta_{f, h}^{N}$ ) to coincide with the Neumann realization on 0 -forms and to preserve the complex structure:

$$
\text { and } \begin{aligned}
\left(1+\Delta_{f, h}^{N,(p+1)}\right)^{-1} d_{f, h}^{(p)} & =d_{f, h}^{(p)}\left(1+\Delta_{f, h}^{N,(p)}\right)^{-1} \\
\left(1+\Delta_{f, h}^{N,(p-1)}\right)^{-1} d_{f, h}^{(p-1), *} & =d_{f, h}^{(p-1), *}\left(1+\Delta_{f, h}^{N,(p)}\right)^{-1}
\end{aligned}
$$

on the form domain of $\Delta_{f, h}^{N,(p)}$.

Having in mind the works [Sch] and [ChLi] about cohomology complexes and boundary problems, we introduce the space:

$$
\Lambda^{p} H_{0, \mathbf{n}}^{1}=H_{0, \mathbf{n}}^{1}\left(\Omega ; \Lambda^{p} T^{*} \Omega\right)=\left\{\omega \in H^{1}\left(\Omega ; \Lambda^{p} T^{*} \Omega\right) ; \mathbf{n} \omega=0\right\} .
$$


In the case $p=0$, it coincides with the space $H^{1}(\Omega)$, while for $p \geq 1$ the condition says only that the form vanishes on $\partial \Omega$ when applied to non tangential $p$-vectors. Since the boundary $\partial \Omega$ is assumed to be regular, the space

$$
\Lambda^{p} \mathcal{C}_{0, \mathbf{n}}^{\infty}=\mathcal{C}_{0, \mathbf{n}}^{\infty}\left(\Omega ; \Lambda^{p} T^{*} \Omega\right)=\left\{\omega \in \mathcal{C}^{\infty}\left(\bar{\Omega}, \Lambda^{p} T^{*} \Omega\right) ; \mathbf{n} \omega=0\right\}
$$

is dense in $\Lambda^{p} H_{0, \mathbf{n}}^{1}$. The next construction is a variant of known results in the case $f=0$ (see $[\mathrm{Sch}]$ ). We will use the notations:

$$
\mathcal{D}_{f, h}(\omega, \eta)=\left\langle d_{f, h} \omega \mid d_{f, h} \eta\right\rangle_{\Lambda^{p+1} L^{2}}+\left\langle d_{f, h}^{*} \omega \mid d_{f, h}^{*} \eta\right\rangle_{\Lambda^{p-1} L^{2}}
$$

and

$$
\mathcal{D}_{f, h}(\omega)=\mathcal{D}_{f, h}(\omega, \omega)=\left\|d_{f, h} \omega\right\|_{\Lambda^{p+1} L^{2}}^{2}+\left\|d_{f, h}^{*} \omega\right\|_{\Lambda^{p-1} L^{2}}^{2} .
$$

\section{Proposition 2.3.1.}

The non negative quadratic form $\omega \rightarrow \mathcal{D}_{f, h}(\omega)$ is closed on $\Lambda^{p} H_{0, \mathbf{n}}^{1}$. The associated (self-adjoint) Friedrichs extension is denoted by $\Delta_{f, h}^{N,(p)}$. Its domain is:

$$
D\left(\Delta_{f, h}^{N,(p)}\right)=\left\{u \in \Lambda^{p} H^{2} ; \quad \mathbf{n} \omega=0 \text { and } \mathbf{n} d_{f, h} \omega=0\right\},
$$

and we have:

$$
\forall \omega \in D\left(\Delta_{f, h}^{N,(p)}\right), \quad \Delta_{f, h}^{N,(p)} \omega=\Delta_{f, h}^{(p)} \omega \quad \text { in } \Omega .
$$

Proof.

By the same argument as in the proof of Proposition 2.4 of [HeNi1], the space $\Lambda^{p} H_{0, \mathbf{n}}^{1}$ is isomorphic to the direct sum:

$$
\Lambda^{p} H_{0}^{1} \oplus \mathbf{t} \Lambda^{p} H^{1 / 2}\left(\partial \Omega ; \Lambda^{p} T^{*} \Omega\right)
$$

with continuous embedding. Hence, since $\partial \Omega$ is a regular boundaryless manifold, its dual is the direct sum of $\Lambda^{p} H^{-1}$ and $\mathbf{t} \Lambda^{p} H^{-1 / 2}\left(\partial \Omega ; \Lambda^{p} T^{*} \Omega\right)$ :

$$
\left(\Lambda^{p} H_{0, \mathbf{n}}^{1}\right)^{\prime}=\Lambda^{p} H^{-1} \oplus \mathbf{t} \Lambda^{p} H^{-1 / 2}\left(\partial \Omega ; \Lambda^{p} T^{*} \Omega\right) .
$$

We have to check that $\omega \mapsto \mathcal{D}_{f, h}^{(p)}(\omega)+C\|\omega\|_{\Lambda^{p} L^{2}}^{2}$ is equivalent to the square of the $\Lambda^{p} H^{1}$ norm on $\Lambda^{p} H_{0, \mathbf{n}}^{1}$. By (2.1.6)-(2.1.9) this is equivalent to the same result for $f=0$ and $h=1$. This last case is known as Gaffney's inequality which is a consequence of the Weitzenböck formula (see [Sch], Theorem 2.1.7).

Hence the quadratic form $\omega \rightarrow \mathcal{D}_{f, h}(\omega)$ is closed on $\Lambda^{p} H_{0, \mathbf{n}}^{1}$ and the identity

$$
\forall \eta \in \Lambda^{p} H_{0, \mathbf{n}}^{1}, \quad \mathcal{D}_{f, h}^{(p)}(\omega, \eta)=\left\langle A^{(p)} \omega, \eta\right\rangle
$$

defines an isomorphism $A^{(p)}: \Lambda^{p} H_{0, \mathbf{n}}^{1} \rightarrow\left(\Lambda^{p} H_{0, \mathbf{n}}^{1}\right)^{\prime}$.

The self-adjoint Friedrichs extension $\Delta_{f, h}^{N,(p)}$ is then defined as the operator:

$$
D\left(\Delta_{f, h}^{N,(p)}\right)=\left\{\omega \in \Lambda^{p} H_{0, \mathbf{n}}^{1}, A^{(p)} \omega \in \Lambda^{p} L^{2}\right\}, \quad \Delta_{f, h}^{N,(p)} \omega=A^{(p)} \omega .
$$


It remains to identify this domain and the explicit action of $A^{(p)}$. If $\omega$ belongs to $D\left(\Delta_{f, h}^{N,(p)}\right)$, by the first Green formula (2.2.7) we get:

$$
\forall \eta \in \Lambda^{p} \mathcal{C}_{0}^{\infty}, \quad\left\langle\omega \mid A^{(p)} \eta\right\rangle=\mathcal{D}_{f, h}^{(p)}(\omega, \eta)=\left\langle\omega \mid \Delta_{f, h}^{(p)} \eta\right\rangle .
$$

The inequality:

$$
\left|\mathcal{D}_{f, h}^{(p)}(\omega, \eta)\right| \leq C\|\omega\|_{\Lambda^{p} H^{1}}\|\eta\|_{\Lambda^{p} H^{1}},
$$

together with the density of $\Lambda^{p} \mathcal{C}_{0}^{\infty}$ in $\Lambda^{p} H_{0}^{1}$ implies that the current $\Delta_{f, h}^{(p)} \omega \in$ $\mathcal{D}^{\prime}\left(\Omega ; \Lambda^{p} T^{*} \Omega\right)$ is indeed the $\Lambda^{p} H^{-1}$ component of $A^{(p)} \omega$.

Assume that $\omega$ belongs to $\Lambda^{p} H_{0, \mathbf{n}}^{1} \cap \Lambda^{p} H^{2}$; then the Green formula (2.2.7) gives:

$$
h \int_{\partial \Omega}(\mathbf{t} \bar{\eta}) \wedge\left(\star \mathbf{n} d_{f, h} \omega\right)=\mathcal{D}_{f, h}^{(p)}(\omega, \eta)-\left\langle\Delta_{f, h}^{(p)} \omega \mid \eta\right\rangle_{\Lambda^{p} L^{2}}, \forall \eta \in \Lambda^{p} H_{0, \mathbf{n}}^{1} .
$$

By density, one can define, for any $\omega$ in $\Lambda^{p} H_{0, \mathbf{n}}^{1}$ such that $\Delta_{f, h}^{(p)} \omega \in \Lambda^{p} L^{2}$, a trace of $\mathbf{n} d_{f, h} \omega$ by the previous identity, observing that the r.h.s. defines an antilinear continuous form with respect to $\eta$. With this generalized definition of $\mathbf{n} d_{f, h}^{(p)} \omega$ we claim that:

$$
D\left(\Delta_{f, h}^{N,(p)}\right)=\left\{\omega \in \Lambda^{p} H_{0, \mathbf{n}}^{1}, \Delta_{f, h}^{(p)} \omega \in \Lambda^{p} L^{2} \text { and } \mathbf{n} d_{f, h}^{(p)} \omega=0\right\} .
$$

The last point consists in observing that the boundary value problem

$$
\Delta_{f, h}^{(p)} u=g, \quad \mathbf{n} u=g_{1}, \quad \mathbf{n} d_{f, h}^{(p)} u=g_{2}
$$

satisfies the Lopatinski-Shapiro conditions. At the principal symbol level ( $h>0$ fixed), these conditions are indeed the same as for

$$
\left(d d^{*}+d^{*} d\right)^{(p)} u=g, \quad \mathbf{n} u=g_{1}, \quad \mathbf{n} d^{(p)} u=g_{2} .
$$

This is checked in [Sch]. Hence any solution to (2.3.2) with $g \in \Lambda^{p} L^{2}$, $g_{1}=g_{2}=0$ belongs to $\Lambda^{p} H^{2}$.

\section{Proposition 2.3.2.}

For any $p \in\{0, \ldots, n\}$, the self-adjoint unbounded operator $\Delta_{f, h}^{N,(p)}$ introduced in Proposition 2.3.1 has a compact resolvent.

Moreover, if $z \in \mathbb{C} \backslash \mathbb{R}_{+}$, the commutation relations

$$
\begin{gathered}
\left(z-\Delta_{f, h}^{N,(p+1)}\right)^{-1} d_{f, h}^{(p)} v=d_{f, h}^{(p)}\left(z-\Delta_{f, h}^{N,(p)}\right)^{-1} v, \\
\text { and } \\
\left(z-\Delta_{f, h}^{N,(p-1)}\right)^{-1} d_{f, h}^{(p-1), *} v=d_{f, h}^{(p-1), *}\left(z-\Delta_{f, h}^{N,(p)}\right)^{-1} v,
\end{gathered}
$$

hold for any $v \in \Lambda^{p} H_{0, \mathbf{n}}^{1}$. 
Proof.

The domain of the operator is contained in $\Lambda^{p} H^{2}$, which is compactly embedded in $\Lambda^{p} L^{2}$, by the Sobolev injections. This yields the first statement. Since $\Lambda^{p} \mathcal{C}_{0, \mathbf{n}}^{\infty}$ is dense in $\Lambda^{p} H_{0, \mathbf{n}}^{1}$, it is sufficient to consider the case when $v \in \Lambda^{p} \mathcal{C}_{0, \mathbf{n}}^{\infty}$. For such a $v$ and for $z \in \mathbb{C} \backslash \mathbb{R}_{+}$, we set:

$$
u=\left(z-\Delta_{f, h}^{N,(p)}\right)^{-1} v .
$$

Due to the ellipticity of the associated boundary problem (the LopatinskiShapiro conditions are verified) $u$ belongs to $\mathcal{C}^{\infty}\left(\bar{\Omega} ; \Lambda^{p} T^{*} \Omega\right)$. The commutation relations (2.1.3) and (2.1.4) can be applied since here $f \in \mathcal{C}^{\infty}(\bar{\Omega} ; \mathbb{R})$ :

$$
\left(z-\Delta_{f, h}^{(p+1)}\right) d_{f, h}^{(p)} u=d_{f, h}^{(p)}\left(z-\Delta_{f, h}^{(p)}\right) u=d_{f, h}^{(p)} v
$$

and

$$
\left(z-\Delta_{f, h}^{(p-1)}\right) d_{f, h}^{(p-1), *} u=d_{f, h}^{(p-1), *}\left(z-\Delta_{f, h}^{(p)}\right) u=d_{f, h}^{(p-1), *} v
$$

Since $u \in D\left(\Delta_{f, h}^{N,(p)}\right)$, we have $\mathbf{n} u=0$ and $\mathbf{n} d_{f, h}^{(p)} u=0$.

Then, $\mathbf{n} d_{f, h} u=0$ and $\mathbf{n} d_{f, h} d_{f, h} u=0$ imply $d_{f, h} u \in D\left(\Delta_{f, h}^{N,(p+1)}\right)$. So by (2.3.3) we have:

$$
d_{f, h}^{(p)}\left(z-\Delta_{f, h}^{N,(p)}\right)^{-1} v=d_{f, h} u=\left(z-\Delta_{f, h}^{N,(p+1)}\right)^{-1} d_{f, h}^{(p)} v .
$$

In order to show the second commutation relation, we first use the relation (2.2.5) which implies:

$$
\mathbf{n} d_{f, h}^{*} u=h d^{*} \mathbf{n} u+\mathbf{n}\left(\mathbf{i}_{\nabla f} u\right)=0 .
$$

For the normal trace of the differential, we write $\left(\Delta_{f, h} u=z u-v\right)$ :

$$
\mathbf{n} d_{f, h}\left(d_{f, h}^{*} u\right)=z \mathbf{n} u-\mathbf{n} v-\mathbf{n} d_{f, h}^{*} d_{f, h} u=-d_{f, h}^{*} \mathbf{n} d_{f, h} u=0 .
$$

Hence $d_{f, h}^{(p-1), *} u$ belongs to $D\left(\Delta_{f, h}^{N,(p-1)}\right)$ and the identity (2.3.4) yields the last commutation relation to show.

Definition 2.3.3. For any Borel subset $E \subset \mathbb{R}$ and $p \in\{0, \ldots, n\}$, we will denote by $1_{E}\left(\Delta_{f, h}^{N,(p)}\right)$ the spectral projection of $\Delta_{f, h}^{N,(p)}$ on $E$.

From Proposition 2.3.2 and Stone's Formula we deduce:

Corollary 2.3.4.

For any Borel subset $E \subset \mathbb{R}$, the identities

$$
\begin{array}{cc} 
& 1_{E}\left(\Delta_{f, h}^{N,(p+1)}\right) d_{f, h}^{(p)} v=d_{f, h}^{(p)} 1_{E}\left(\Delta_{f, h}^{N,(p)}\right) v \\
\text { and } & \\
& 1_{E}\left(\Delta_{f, h}^{N,(p-1)}\right) d_{f, h}^{(p-1), *} v=d_{f, h}^{(p-1), *} 1_{E}\left(\Delta_{f, h}^{N,(p)}\right) v
\end{array}
$$


hold for all $v \in \Lambda^{p} H_{0, \mathbf{n}}^{1}$.

In the particular case when $v$ is an eigenvector of $\Delta_{f, h}^{N,(p)}$ corresponding to the eigenvalue $\lambda$, then $d_{f, h}^{(p)} v$ (resp. $d_{f, h}^{(p-1), *} v$ ) belongs to the spectral subspace $\operatorname{Ran} 1_{\{\lambda\}}\left(\Delta_{f, h}^{N,(p+1)}\right)$ (resp. Ran $\left.1_{\{\lambda\}}\left(\Delta_{f, h}^{N,(p-1)}\right)\right)$.

Proposition 2.3.2 and Corollary 2.3.4 were stated for $p$-forms $v \in \Lambda^{p} H_{0, \mathbf{n}}^{1}(\Omega)$, belonging to the form domain of $\Delta_{f, h}^{N,(p)}$. It is convenient to work in this framework because the multiplication by any cut-off function preserves the form domain $\Lambda H_{0, \mathbf{n}}^{1}(\Omega)$ :

$$
\left(\omega \in \Lambda H_{0, \mathbf{n}}^{1}(\Omega), \quad \chi \in \mathcal{C}^{\infty}(\bar{\Omega})\right) \Rightarrow\left(\chi \omega \in \Lambda H_{0, \mathbf{n}}^{1}(\Omega)\right),
$$

while this property is no more true for $D\left(\Delta_{f, h}^{N}\right)$. In this spirit, we will often refer to the next easy consequence of the spectral theorem.

\section{Lemma 2.3.5.}

Let $A$ be a non negative self-adjoint operator on a Hilbert space $\mathcal{H}$ with associated quadratic form $q_{A}(x)=(x \mid A x)$ and with form domain $Q(A)$. Then for any $a, b \in(0,+\infty)$, the implication

$$
\left(q_{A}(u) \leq a\right) \Rightarrow\left(\left\|1_{[b,+\infty)}(A) u\right\|^{2} \leq \frac{a}{b}\right)
$$

holds for any $u \in Q(A)$.

\section{First localization of the spectrum}

\subsection{Introduction and result}

Let us first recall that we are working with the fixed Riemannian metric $g_{0}$ on $\bar{\Omega}$. Like in the third section of [HeNi1] for their tangential Dirichlet realization of the Witten Laplacian, we check here that the number of eigenvalues of $\Delta_{f, h}^{N,(p)}$ smaller than $h^{3 / 2}$ equals a Morse index which involves in its definition the boundary conditions. To this end, we will adapt [HeNi1] which uses techniques yet presented in [Sim2], [CFKS], [ChLi], [Bis], [Bur], and in [Hel1].

In order to make the connection between the normal Neumann realization of the Witten Laplacian $\Delta_{f, h}^{N}$ and the Morse theory, we assume additional properties for the function $f$ up to the boundary $\partial \Omega$.

\section{Assumption 3.1.1.}

The real-valued function $f \in \mathcal{C}^{\infty}(\bar{\Omega})$ is a Morse function on $\Omega$ with no critical points in $\partial \Omega$. In addition its restriction $\left.f\right|_{\partial \Omega}$ is a Morse function on $\partial \Omega$. 


\section{Remark 3.1.2.}

With this assumption, the function $f$ has a finite number of critical points with index $p$ in $\Omega$. Note furthermore that the assumption ensures that there is no critical point on $\partial \Omega$, which implies that the outgoing normal derivative $\frac{\partial f}{\partial n}(U)$ is not 0 when $U$ is a critical point of $\left.f\right|_{\partial \Omega}$.

\section{Definition 3.1.3.}

For $\ell \in\{0, \ldots, n\}$, the integer $m_{\ell,-}^{\partial \Omega}$ is the number of critical points $U$ of $\left.f\right|_{\partial \Omega}$ with index $\ell$ such that $\frac{\partial f}{\partial n}(U)<0$ (with the additional convention $m_{n,-}^{\partial \Omega}=0$ ). For $p \in\{0, \ldots, n\}$, let

$$
m_{p}^{\bar{\Omega}}=m_{p}^{\Omega}+m_{p,-}^{\partial \Omega} .
$$

\section{Remark 3.1.4.}

In [HeNi1], the authors worked with the tangential Dirichlet conditions $\left(\mathbf{t} \omega=0\right.$ and $\left.\mathbf{t} d_{f, h}^{*} \omega=0\right)$ and the corresponding definition was similar with $m_{\ell,-}^{\partial \Omega}$ and $\frac{\partial f}{\partial n}(U)<0$ replaced respectively by $m_{\ell-1,-}^{\partial \Omega}$ and $\frac{\partial f}{\partial n}(U)>0$.

The aim of this section is to prove the following theorem:

\section{Theorem 3.1.5.}

Under Assumption 3.1.1, there exists $h_{0}>0$, such that the normal Neumann realization of the Witten Laplacian $\Delta_{f, h}^{N}$ introduced in Subsection 2.3 has, for $h \in\left(0, h_{0}\right]$, the following property:

For any $p \in\{0, \ldots, n\}$, the spectral subspace $F^{(p)}=\operatorname{Ran}_{\left[0, h^{3 / 2}\right)}\left(\Delta_{f, h}^{N,(p)}\right)$ has rank: $\operatorname{dim} F^{(p)}=m_{p}^{\bar{\Omega}}$.

To prove this theorem, we will adapt for the normal Neumann realization of the Witten Laplacian the proof given in [HeNi1] for the tangential Dirichlet realization. Many points of this demonstration do not require any modification, so we will only recall these results without any demonstration.

The theorem will be proved in the Subsection 3.3.

\subsection{A few preliminary lemmas}

In this subsection, we recall some results of [HeNi1] that we need to prove Theorem 3.1.5.

\subsubsection{Variationnal results for the Witten Laplacian on $\mathbb{R}^{k}$}

Let $g$ be a $\mathcal{C}^{\infty}$ metric on $\mathbb{R}^{k}$ which equals the Euclidean metric outside a compact set $K$.

Assumption 3.2.1 $(g)$.

The function $f$ is a Morse $\mathcal{C}^{\infty}$ real-valued function and there exist $C_{1}>0$ and a compact $K$ such that, for the metric $g$ :

$$
\forall x \in \mathbb{R}^{k} \backslash K, \quad|\nabla f(x)| \geq C_{1}^{-1} \quad \text { and } \quad|\operatorname{Hess} f(x)| \leq C_{1}|\nabla f(x)|^{2} .
$$


Note that the above assumption ensures that $f$ has a finite number of critical points and $m_{p}$ will denote the number of critical points with index $p$. Let us recall the Propositions 3.6 and 3.7 of [HeNi1]. They gather consequences of Simader's Theorem in [Sima] about the essential self-adjointness of non negative Schrödinger operators, of Persson's Lemma in [Per] about the localization of the essential spectrum and of the semiclassical analysis a la Witten in [Wit] leading to Morse inequalities. We refer the reader also to [CFKS] [Hen] [Hel3] or [Zha] for the Witten approach to Morse inequalities in the boundaryless case and to [Mil1] and [Lau] for a topological presentation of Morse theory.

\section{Proposition 3.2.2.}

Under Assumption 3.2.1, there exist $h_{0}>0, c_{0}>0$ and $c_{1}>0$ such that the following properties are satisfied for any $h \in\left(0, h_{0}\right]$ :

i) The Witten Laplacian $\Delta_{f, h}$ as an unbounded operator on $L^{2}\left(\mathbb{R}^{k} ; \Lambda T^{*} \mathbb{R}^{k}\right)$ is essentially self-adjoint on $\mathcal{C}_{0}^{\infty}\left(\mathbb{R}^{k} ; \Lambda T^{*} \mathbb{R}^{k}\right)$.

ii) For any Borel subset $E$ in $\mathbb{R}$, the identities

$$
\text { and } \begin{aligned}
1_{E}\left(\Delta_{f, h}^{(p+1)}\right) d_{f, h}^{(p)} u & =d_{f, h}^{(p)} 1_{E}\left(\Delta_{f, h}^{(p)}\right) u \\
1_{E}\left(\Delta_{f, h}^{(p-1)}\right) d_{f, h}^{(p-1), *} u & =d_{f, h}^{(p-1), *} 1_{E}\left(\Delta_{f, h}^{(p)}\right) u
\end{aligned}
$$

hold for any $u$ belonging to the form domain of $\Delta_{f, h}^{(p)}$.

In particular, if $v$ is an eigenvector of $\Delta_{f, h}^{(p)}$ associated with the eigenvalue $\lambda$, then $d_{f, h}^{(p)} v\left(\right.$ resp. $\left.d_{f, h}^{(p-1), *} v\right)$ belongs to the spectral subspace $\operatorname{Ran} 1_{\{\lambda\}}\left(\Delta_{f, h}^{(p+1)}\right)$ (resp. Ran $1_{\{\lambda\}}\left(\Delta_{f, h}^{(p-1)}\right)$ ).

iii) The essential spectrum $\sigma_{e s s}\left(\Delta_{f, h}^{(p)}\right)$ is contained in $\left[c_{1},+\infty\right)$.

iv) The range of $1_{\left[0, c_{0} h\right)}\left(\Delta_{f, h}^{(p)}\right)$ has dimension $m_{p}$, for all $h \in\left(0, h_{0}\right]$.

\section{Proposition 3.2.3.}

If the Morse function $f$ satisfies Assumption 3.2.1 and admits a unique critical point at $x=0$ with index $p_{0}$, so $m_{p}=\delta_{p, p_{0}}$, then there exist $h_{0}>0$ and $c_{0}>0$, such that the following properties hold for $h \in\left(0, h_{0}\right]$ :

i) For $p \neq p_{0}, \Delta_{f, h}^{(p)} \geq c_{0} h \mathrm{Id}$.

ii) If $\psi_{p_{0}}^{h}$ is a normalized eigenvector of the one dimensional spectral subspace Ran $1_{\left[0, c_{0} h\right)}\left(\Delta_{f, h}^{\left(p_{0}\right)}\right)$, it satisfies

$$
d_{f, h} \psi_{p_{0}}^{h}=0, \quad d_{f, h}^{\left(p_{0}-1\right), *} \psi_{p_{0}}^{h}=0 \quad \text { and } \quad \Delta_{f, h}^{\left(p_{0}\right)} \psi_{p_{0}}^{h}=0
$$

so that Ran $1_{\left[0, c_{0} h\right)}\left(\Delta_{f, h}^{\left(p_{0}\right)}\right)=\operatorname{Ker} \Delta_{f, h}^{\left(p_{0}\right)}$. Moreover

$$
\sigma\left(\Delta_{f, h}^{\left(p_{0}\right)}\right) \backslash\{0\} \subset\left[c_{0} h, \infty\right) .
$$


iii) If $\chi \in \mathcal{C}_{0}^{\infty}\left(\mathbb{R}^{k}\right)$ satisfies $\chi=1$ in a neighborhood of 0 , then there exists $C_{\chi} \geq 1$, such that, for all $h \in\left(0, h_{0} / C_{\chi}\right)$, the inequality,

$$
(1-\chi) \Delta_{f, h}^{(p)}(1-\chi) \geq C_{\chi}^{-1}[1-\chi]^{2},
$$

holds in the sense of quadratic form on $\Lambda^{p} H^{1}\left(\mathbb{R}^{k}\right)$.

\subsubsection{The model half-space problem}

We work here on $\mathbb{R}_{-}^{n}=\mathbb{R}^{n-1} \times(-\infty, 0)$ with a Riemannian metric $\tilde{g}_{0}$. Assume furthermore that there are coordinates $x=\left(x^{\prime}, x_{n}\right)$ such that $\tilde{g}_{0}=$ $\sum_{i, j=1}^{n} \tilde{g}_{i j}^{0}(x) d x_{i} d x_{j}$ satisfies

$$
\tilde{g}_{i, n}^{0}=\tilde{g}_{n, i}^{0}=0 \quad \text { for } i<n
$$

and

$$
\forall x \in \overline{\mathbb{R}_{-}^{n}} \backslash K_{1}, \quad \partial_{x} \tilde{g}_{i j}^{0}(x)=0,
$$

for some compact set $K_{1} \subset \overline{\mathbb{R}_{-}^{n}}$.

In this paragraph, the coordinates $\left(x^{\prime}, x_{n}\right)$ are fixed while different metrics on $\overline{\mathbb{R}_{-}^{n}}$ are considered. The notation $G(\cdot)$ will be used for the matrix valued map $x \mapsto G(x)={ }^{t} G(x)=\left(g_{i j}(x)\right)_{i, j} \in G L^{n}(\mathbb{R})$, which is assumed to be a $\mathcal{C}^{\infty}$ function. According to the standard notation, the coefficients of $G(x)^{-1}$ are written $g^{i j}(x)$.

Consider also a function $f$ which has a specific form in the same coordinates $\left(x^{\prime}, x_{n}\right)$.

\section{Assumption 3.2.4.}

The function $f \in \mathcal{C}^{\infty}\left(\overline{\mathbb{R}_{-}^{n}}\right)$ satisfies:

i) The estimates $|\nabla f(x)| \geq C^{-1} \quad$ and $\quad\left|\partial_{x}^{\alpha} f(x)\right| \leq C_{\alpha}$ hold, for all $x \in$ $\overline{\mathbb{R}_{-}^{n}}$ and all $\alpha \in \mathbb{N}^{n}, \alpha \neq 0$.

ii) The function $f$ is the sum $f\left(x^{\prime}, x_{n}\right)=-\frac{1}{2} f_{+}\left(x_{n}\right)+\frac{1}{2} f_{-}\left(x^{\prime}\right)$. Moreover, there exists $C_{1}>0$ such that

$$
\forall x_{n} \in(-\infty, 0), \quad C_{1}^{-1} \leq\left|\partial_{x_{n}} f_{+}\left(x_{n}\right)\right| \leq C_{1},
$$

and $f_{-}$is a Morse function on $\mathbb{R}^{n-1}$ which satisfies Assumption 3.2.1 for the metric $\sum_{i, j=1}^{n-1} \tilde{g}_{i j}^{0}\left(x^{\prime}, 0\right) d x_{i} d x_{j}$ and admits a unique critical point at $x^{\prime}=0$ with index $p_{0}$.

The boundedness of $\left|\partial_{x}^{\alpha} f\right|, 1 \leq|\alpha| \leq 2$, avoids any subtle questions about the domains. 


\section{Proposition 3.2.5.}

Under Assumption 3.2.4-i), the unbounded operator $\Delta_{f, h}^{N}$ on $L^{2}\left(\mathbb{R}_{-}^{n} ; \Lambda T^{*} \mathbb{R}_{-}^{n}\right)$, with domain

$$
D\left(\Delta_{f, h}^{N}\right)=\left\{\omega \in \Lambda H^{2}\left(\mathbb{R}_{-}^{n}\right), \quad \mathbf{n} \omega=0, \quad \mathbf{n} d_{f, h} \omega=0\right\},
$$

is self-adjoint.

If $E$ is any Borel subset of $\mathbb{R}$, the relations

$$
\text { and } \begin{aligned}
1_{E}\left(\Delta_{f, h}^{N,(p+1)}\right) d_{f, h}^{(p)} u & =d_{f, h}^{(p)} 1_{E}\left(\Delta_{f, h}^{N,(p)}\right) u, \\
1_{E}\left(\Delta_{f, h}^{N,(p-1)}\right) d_{f, h}^{(p-1), *} u & =d_{f, h}^{(p-1), *} 1_{E}\left(\Delta_{f, h}^{N,(p)}\right) u,
\end{aligned}
$$

hold for any $u \in \Lambda^{p} H_{0, \mathbf{n}}^{1}\left(\mathbb{R}_{-}^{n}\right)$.

Proof.

The uniform estimate on $\nabla f$ allows the same proof as for Proposition 2.3.2 and Corollary 2.3.4 (here $\mathcal{C}_{0, \mathbf{n}}^{\infty}$ denotes the space of $\mathcal{C}^{\infty}$ compactly supported functions in $\overline{\mathbb{R}_{-}^{n}}$ with a vanishing normal component on $\left\{x_{n}=0\right\}$ ).

We are looking for a result similar to Proposition 3.2.2 and Proposition 3.2.3 for the case with normal boundary condition on $\mathbb{R}_{-}^{n}$ (this result will be stated in Subsection 3.2.3). One difficulty here comes from the metric which, although diagonal in the coordinates $\left(x^{\prime}, x_{n}\right)$, is not constant. The general case can be reduced to a simpler situation where $g_{i j}(x)=g_{i j}\left(x^{\prime}\right)$ with $g_{n n}=$ 1 after several steps.

We need some notations.

\section{Definition 3.2.6.}

For a metric $g$ which satisfies (3.2.4), the corresponding $H^{s}$-norm on the space $\Lambda^{p} H^{s}\left(\overline{\mathbb{R}_{-}^{n}}\right)$ is denoted by \|\|$_{\Lambda^{p} H^{s}, g}$ and the notation \|\|$_{\Lambda^{p} H^{s}}$ is kept for the Euclidean metric $g_{e}=\sum_{i=1}^{n} d x_{i}^{2}$.

Similarly, the quadratic form associated with $\Delta_{f, h}^{N,(p)}$ is written

$$
\mathcal{D}_{g, f, h}(\omega)=\left\|d_{g, f, h}^{*} \omega\right\|_{\Lambda^{p-1} L^{2}, g}^{2}+\left\|d_{f, h} \omega\right\|_{\Lambda^{p+1} L^{2}, g}^{2}, \quad \forall \omega \in \Lambda^{p} H_{0, \mathbf{n}}^{1}\left(\mathbb{R}_{-}^{n}\right),
$$

where the codifferential $d_{g, f, h}^{*}$ also depends on $g$.

Remark 3.2.7. The considered metrics satisfying (3.2.4), the different $\left(L^{2}, g\right)$-norms are equivalent.

The required accuracy while comparing the quadratic forms $\mathcal{D}_{g, f, h}$ needs some care.

We will work further with partitions of unity and the next proposition, similar to the standard IMS localization formula (see $[\mathrm{CFKS}]$ ), but in the case with boundary, will be useful. 
Proposition 3.2.8. (IMS Localization Formula)

For $W=\Omega$ or $W=\mathbb{R}_{-}^{n}$, consider $\left\{\chi_{k}\right\}_{1, \ldots, N}$ a partition of unity of $\bar{W}$ (i.e. satisfying $\sum_{k=1}^{N} \chi_{k}^{2}=1$ on $\left.\bar{W}\right)$.

Let $g$ and $f$ be respectively a Riemannian metric and a $\mathcal{C}^{\infty}$ function (satisfying Assumption 3.2.4-i) in the case $\mathbb{R}_{-}^{n}$ ) on $\bar{W}$.

The next IMS localization formula is then valid:

$$
\forall \omega \in \Lambda H_{0, \mathbf{n}}^{1}, \quad \mathcal{D}_{g, f, h}(\omega)=\sum_{k=1}^{N} \mathcal{D}_{g, f, h}\left(\chi_{k} \omega\right)-h^{2}\left\|\left|\nabla \chi_{k}\right| \omega\right\|_{\Lambda L^{2}, g}^{2} .
$$

Proof. For clarity, we omit the dependence on $g$ in the proof.

Recall, from $\sum_{k=1}^{N} \chi_{k}^{2}=1$, than for any $\eta \in \Lambda H^{1}$ :

$$
\sum_{k=1}^{N} \chi_{k} d \chi_{k} \wedge \eta=0, \quad \text { and by duality (2.1.5), } \quad \sum_{k=1}^{N} \chi_{k} \mathbf{i}_{\nabla \chi_{k}} \eta=0 .
$$

Now, for any $\omega \in \Lambda H_{0, \mathbf{n}}^{1}$ and $k \in\{1, \ldots, N\}$,

$$
\mathcal{D}_{f, h}\left(\chi_{k} \omega\right)=\left\|d_{f, h}\left(\chi_{k} \omega\right)\right\|+\left\|d_{f, h}^{*}\left(\chi_{k} \omega\right)\right\| .
$$

From (2.1.6) and (2.1.7),

$$
d_{f, h}\left(\chi_{k} \omega\right)=h d \chi_{k} \wedge \omega+\chi_{k} d_{f, h} \omega \quad \text { and } \quad d_{f, h}^{*}\left(\chi_{k} \omega\right)=h \mathbf{i}_{\nabla \chi_{k}} \omega+\chi_{k} d_{f, h}^{*} \omega .
$$

Hence, from $\sum_{k=1}^{N} \chi_{k}^{2}=1,(2.1 .6)$, and (2.1.7), for any $\omega \in \Lambda H_{0, \mathbf{n}}^{1}$,

$$
\begin{gathered}
\sum_{k=1}^{N} \mathcal{D}_{f, h}\left(\chi_{k} \omega\right)=\mathcal{D}_{f, h}(\omega)+\sum_{k=1}^{N} h^{2}\left(\left\langle d \chi_{k} \wedge \omega \mid d \chi_{k} \wedge \omega\right\rangle+\left\langle\mathbf{i}_{\nabla \chi_{k}} \omega \mid \mathbf{i}_{\nabla \chi_{k}} \omega\right\rangle\right) \\
+\sum_{k=1}^{N} 2 \operatorname{Re}\left(\left\langle h d \chi_{k} \wedge \omega \mid h \chi_{k} d \omega+\chi_{k} d f \wedge \omega\right\rangle+\left\langle h \mathbf{i}_{\nabla \chi_{k}} \omega \mid h \chi_{k} d^{*} \omega+\chi_{k} \mathbf{i}_{\nabla f} \omega\right\rangle\right) .
\end{gathered}
$$

Using (3.2.7),

$$
\sum_{k=1}^{N} \mathcal{D}_{f, h}\left(\chi_{k} \omega\right)=\mathcal{D}_{f, h}(\omega)+h^{2} \sum_{k=1}^{N}\left(\left\langle d \chi_{k} \wedge \omega \mid d \chi_{k} \wedge \omega\right\rangle+\left\langle\mathbf{i}_{\nabla \chi_{k}} \omega \mid \mathbf{i}_{\nabla \chi_{k}} \omega\right\rangle\right) .
$$

At least, the identity

$$
\mathbf{i}_{X}(\alpha \wedge \beta)=\left(\mathbf{i}_{X} \alpha\right) \wedge \beta+(-1)^{\operatorname{deg} \alpha} \alpha \wedge\left(\mathbf{i}_{X} \beta\right)
$$

implies

$$
\begin{gathered}
\left\langle d \chi_{k} \wedge \omega \mid d \chi_{k} \wedge \omega\right\rangle+\left\langle\mathbf{i}_{\nabla \chi_{k}} \omega \mid \mathbf{i}_{\nabla \chi_{k}} \omega\right\rangle=\left\langle\mathbf{i}_{\nabla \chi_{k}}\left(d \chi_{k} \wedge \omega\right)+d \chi_{k} \wedge\left(\mathbf{i}_{\nabla \chi_{k}} \omega\right) \mid \omega\right\rangle \\
=\left\langle\left(\mathbf{i}_{\nabla \chi_{k}} d \chi_{k}\right) \omega \mid \omega\right\rangle=\left\langle\left|\nabla \chi_{k}\right|^{2} \omega \mid \omega\right\rangle,
\end{gathered}
$$

which proves the proposition. 
Let us give now two lemmas whose proofs are the same than the proofs of Lemmas 3.11 and 3.12 of [HeNi1].

The first lemma provides a reduction to the case $\partial_{x_{n}} G=0$ and the second allows us to consider again a simpler metric with $g_{n n}=1$.

\section{Lemma 3.2.9.}

Let $g_{1}$ and $g_{2}$ be two metrics which satisfy (3.2.4) and coincide on $\left\{x_{n}=0\right\}$. Let $f$ be a function satisfying Assumption 3.2.4. There exist constants $C_{12} \geq$ 1 and $h_{0}>0$ such that the inequality,

$$
\mathcal{D}_{g_{2}, f, h}(\omega) \geq\left(1-C_{12} h^{2 / 5}\right) \mathcal{D}_{g_{1}, f, h}(\omega)-C_{12} h^{7 / 5}\|\omega\|_{\Lambda^{p} L^{2}, g_{1}}^{2},
$$

holds for $\omega \in \Lambda^{p} H_{0, \mathbf{n}}^{1}\left(\mathbb{R}_{-}^{n}\right)$, with $p \in\{0, \ldots, n\}$ and $h \in\left(0, h_{0}\right)$, as soon as $\operatorname{supp} \omega \subset\left\{x_{n} \geq-C_{0} h^{2 / 5}\right\}$.

\section{Lemma 3.2.10.}

Let $g_{1}$ and $g_{2}$ be two conformal metrics (which satisfy (3.2.4)) in the sense:

$$
g_{2}=e^{\varphi(x)} g_{1} .
$$

Let $f$ be a function satisfying Assumption 3.2.4. Then there exist constants $C_{12} \geq 1$ and $h_{0}>0$, such that the inequality,

$$
\forall \omega \in \Lambda^{p} H_{0, \mathbf{n}}^{1}\left(\mathbb{R}_{-}^{n}\right), \quad \mathcal{D}_{g_{2}, f, h}(\omega) \geq C_{12}^{-1} \mathcal{D}_{g_{1}, f, h}(\omega)-C_{12} h^{2}\|\omega\|_{\Lambda^{p} L^{2}, g_{1}}^{2},
$$

holds, for all $p \in\{0, \ldots, n\}$ and all $h \in\left(0, h_{0}\right)$.

\subsubsection{Small eigenvalues for the model half-space problem}

Before giving the proof of Theorem 3.1.5, we state the main result for the model half-space problem which is similar to Proposition 3.2.2 and Proposition 3.2.3.

\section{Proposition 3.2.11.}

Assume that the metric $\tilde{g}_{0}$ satisfies (3.2.3) and (3.2.4) and let $f$ be a Morse function satisfying Assumption 3.2.4 for some $p_{0} \in\{0, \ldots, n\}$. Then there exist constants $h_{0}>0, c_{0}>0$ and $c_{1}>0$, such that the self-adjoint operator $\Delta_{f, h}^{N}$ satisfies the following properties for $h \in\left(0, h_{0}\right]$ :

i) For $p \in\{0, \ldots, n\}$, the essential spectrum $\sigma_{e s s}\left(\Delta_{f, h}^{N,(p)}\right)$ is contained in $\left[c_{1},+\infty\right)$.

ii) For $p \in\{0, \ldots, n\}$, the range of $1_{\left[0, c_{0} h\right)}\left(\Delta_{f, h}^{N,(p)}\right)$ has dimension

$$
\begin{cases}\delta_{p, p_{0}} & \text { if } \quad \partial_{x_{n}} f(0)=-\frac{1}{2} \partial_{x_{n}} f_{+}(0)<0, \\ 0 & \text { if } \quad \partial_{x_{n}} f(0)=-\frac{1}{2} \partial_{x_{n}} f_{+}(0)>0 .\end{cases}
$$

iii) In the first case,

$$
\operatorname{Ran} 1_{\left[0, c_{0} h\right)}\left(\Delta_{f, h}^{N,\left(p_{0}\right)}\right)=\operatorname{Ker} \Delta_{f, h}^{N,\left(p_{0}\right)}=\mathbb{C} \varphi^{h},
$$


where

$$
\left\|\varphi^{h}-\left(e^{f_{+}\left(x_{n}\right) / 2 h}\right) \psi_{p_{0}}^{h}\right\|_{\Lambda^{p} L^{2}}=\mathcal{O}\left(h^{1 / 10}\right),
$$

and $\psi_{p_{0}}^{h}$ belongs to the kernel of a $(n-1)$-dimensional Witten Laplacian $\Delta_{g^{\prime}, f_{-} / 2, h}^{\left(p_{0}\right)}$ in a metric $g^{\prime}$, which is conformal to $\tilde{g}_{0}^{\prime}=\sum_{i, j=1}^{n-1} \tilde{g}_{i j}^{0}\left(x^{\prime}, 0\right) d x_{i} d x_{j}$ on $\mathbb{R}^{n-1}$.

iv) For any $\chi \in \mathcal{C}_{0}^{\infty}\left(\overline{\mathbb{R}_{-}^{n}}\right)$ such that $\chi=1$ in a neighborhood of 0 , there exists $C_{\chi}>0$ such that the lower bounds

$$
(1-\chi) \Delta_{f, h}^{N,(p)}(1-\chi) \geq C_{\chi}^{-1}[1-\chi]^{2}, 0 \leq p \leq n,
$$

hold, for any $h \in\left(0, h_{0} / C_{\chi}\right)$, in the sense of quadratic forms on $\Lambda^{p} H_{0, \mathbf{n}}^{1}\left(\mathbb{R}_{-}^{n}\right)$.

\section{Remark 3.2.12.}

This proposition is an adaptation of Proposition 3.13 of [HeNi1] in the case with normal boundary conditions: we have mainly replaced $f_{+}\left(x_{n}\right)$ by $-f_{+}\left(x_{n}\right)$ and $p_{0}+1$ by $p_{0}$ and the proof is similar.

Proof.

The clue of this result is an accurate lower bound for the quadratic form $\mathcal{D}_{\tilde{g}_{0}, f, h}(\eta)$, when evaluated for $\eta$ such that supp $\eta \subset\left\{x_{n} \geq-C_{0} h^{2 / 5}\right\}$. By Lemmas 3.2.9 and 3.2.10, one can find a metric $g$, which satisfies (3.2.3) and (3.2.4), with $G(x)=G\left(x^{\prime}\right)$ independent of the $x_{n}$-coordinate, $g_{n n}=1$ and a constant $C>1$ such that

$$
\mathcal{D}_{\tilde{g}_{0}, f, h}(\eta) \geq C^{-1} \mathcal{D}_{g, f, h}(\eta)-C h^{7 / 5}\|\eta\|_{\Lambda L^{2}, g}^{2} .
$$

Take two cut-off functions $\tilde{\chi}_{i} \in \mathcal{C}^{\infty}(\mathbb{R})$, such that $\tilde{\chi}_{1} \in \mathcal{C}_{0}^{\infty}(\mathbb{R}), \tilde{\chi}_{1}=1$ in a neighborhood of 0 such that $\tilde{\chi}_{1}^{2}+\tilde{\chi}_{2}^{2}=1$.

By the IMS localization formula (3.2.6), for any $\omega \in \Lambda H_{0, \mathbf{n}}^{1}\left(\mathbb{R}_{-}^{n}\right)$,

$$
\begin{aligned}
\mathcal{D}_{\tilde{g}_{0}, f, h}(\omega) \geq \mathcal{D}_{\tilde{g}_{0}, f, h}\left(\tilde{\chi}_{1}\left(h^{-2 / 5} x_{n}\right) \omega\right)+\mathcal{D}_{\tilde{g}_{0}, f, h}\left(\tilde{\chi}_{2}\left(h^{-2 / 5} x_{n}\right) \omega\right) & \\
& -C h^{6 / 5}\|\omega\|_{\Lambda L^{2}, \tilde{g}_{0}}^{2}
\end{aligned}
$$

By (2.2.9), since $|\nabla f(x)|^{2} \geq C^{-1}$ on $\mathbb{R}_{-}^{n}$, the second term of the r.h.s. is bounded from below by a constant times $\left.\| \tilde{\chi}_{2}\left(h^{-2 / 5} x_{n}\right)\right) \omega \|_{\Lambda L^{2}, \tilde{g}_{0}}^{2}$ and we get:

$$
\begin{aligned}
\mathcal{D}_{\tilde{g}_{0}, f, h}(\omega) \geq \mathcal{D}_{\tilde{g}_{0}, f, h}\left(\tilde{\chi}_{1}\left(h^{-2 / 5} x_{n}\right) \omega\right)-C h^{6 / 5}\left\|\tilde{\chi}_{1}\left(h^{-2 / 5} x_{n}\right) \omega\right\|_{\Lambda L^{2}, \tilde{g}_{0}}^{2} \\
+\frac{C^{-1}}{2}\left\|\tilde{\chi}_{2}\left(h^{-2 / 5} x_{n}\right) \omega\right\|_{\Lambda L^{2}, \tilde{g}_{0}}^{2}
\end{aligned}
$$


Finally after changing the constant $C \geq 1$, the inequality (3.2.10) yields

$$
\begin{array}{r}
\mathcal{D}_{\tilde{g}_{0}, f, h}(\omega) \geq C^{-1} \mathcal{D}_{g, f, h}\left(\tilde{\chi}_{1}\left(h^{-2 / 5} x_{n}\right) \omega\right) \\
-C h^{6 / 5}\left\|\tilde{\chi}_{1}\left(h^{-2 / 5} x_{n}\right) \omega\right\|^{2} \\
+C^{-1}\left\|\tilde{\chi}_{2}\left(h^{-2 / 5} x_{n}\right) \omega\right\|^{2}
\end{array}
$$

where the $L^{2}$-norms in the r.h.s. can be computed with the metric $g$ or $\tilde{g}_{0}$ while possibly adapting the constant $C$, owing to Remark 3.2.7. Here and in the sequel, we omit the subscript $\left(\Lambda L^{2}, g\right)$ for $L^{2}$-norms.

Now the problem is reduced to the analysis of $\mathcal{D}_{g, f, h}$ with the metric $g$. The product structure of the metric $g$ allows an explicit analysis of the spectrum.

(a) The case $n=1$.

We have $x=x_{n} \in \mathbb{R}_{-}, f(x)=-\frac{1}{2} f_{+}\left(x_{n}\right)$. Here the metric is $g=d x_{n}^{2}$. We keep the reference to the index $n$ for the later application.

The spaces $\Lambda^{0} H_{0, \mathbf{n}}^{1}\left(\mathbb{R}_{-}\right)$and $\Lambda^{1} H_{0, \mathbf{n}}^{1}\left(\mathbb{R}_{-}\right)$are respectively $H^{1}\left(\mathbb{R}_{-}\right)$and $\left\{\beta\left(x_{n}\right) d x_{n}, \beta \in H_{0}^{1}\left(\mathbb{R}_{-}\right)\right\}$.

By identity (2.2.9), for any 1 -form $\beta d x_{n}$ with $\beta \in H_{0}^{1}\left(\mathbb{R}_{-}\right)$:

$$
\mathcal{D}_{g,-f_{+} / 2, h}\left(\beta d x_{n}\right)=h^{2}\left\|\partial_{x_{n}} \beta\right\|^{2}+\frac{1}{4}\left\|\partial_{x_{n}} f_{+} \beta\right\|^{2}-\frac{h}{2}\left\langle\partial_{x_{n}}^{2} f_{+}\left(x_{n}\right) \beta \mid \beta\right\rangle .
$$

From (3.2.12), we get:

$$
\mathcal{D}_{g,-f_{+} / 2, h}\left(\beta d x_{n}\right) \geq\left(C^{-2}-h C\right)\|\beta\|^{2},
$$

and deduce that there exist $c_{1}\left(\partial_{x_{n}} f_{+}, \partial_{x_{n}}^{2} f_{+}\right)=c_{1}>0$ and $h_{0}>0$ such that, for all $h \in\left(0, h_{0}\right]$,

$$
\Delta_{g,-f_{+} / 2, h}^{N,(1)} \geq c_{1} \mathrm{Id} .
$$

Again by identity (2.2.9), we have for any 0 -form $\alpha \in H^{1}\left(\mathbb{R}_{-}\right)$:

$$
\begin{array}{r}
\mathcal{D}_{g,-f_{+} / 2, h}(\alpha)=h^{2}\left\|\partial_{x_{n}} \alpha\right\|^{2}+\frac{1}{4}\left\|\partial_{x_{n}} f_{+} \alpha\right\|^{2} \\
+\frac{h}{2}\left\langle\partial_{x_{n}}^{2} f_{+}\left(x_{n}\right) \alpha \mid \alpha\right\rangle \\
-\frac{h}{2} \partial_{x_{n}} f_{+}(0)|\alpha(0)|^{2},
\end{array}
$$

and there are two subcases:

(a1) Subcase $\partial_{x_{n}} f_{+}(0)<0$ :

In this case, identity (3.2.14) implies:

$$
\forall \alpha \in \Lambda^{0} H_{0, \mathbf{n}}^{1}, \quad \mathcal{D}_{g,-f_{+} / 2, h}(\alpha) \geq\left(C^{-2}-h C\right)\|\alpha\|^{2},
$$


which provides the existence of $c_{1}\left(\partial_{x_{n}} f_{+}, \partial_{x_{n}}^{2} f_{+}\right)=c_{1}>0$ and $h_{0}>0$ such that:

$$
\Delta_{-f_{+} / 2, h}^{N,(0)} \geq c_{1} \mathrm{Id}, \forall h \in\left(0, h_{0}\right] .
$$

(a2) Subcase $\partial_{x_{n}} f_{+}(0)>0$ :

If $\Delta_{-f_{+} / 2, h}^{N,(0)}(\alpha)=\lambda_{h} \alpha$, with $\lambda_{h}<c_{1}$, we have by Proposition 3.2.5(3.2.5):

$$
\Delta_{-f_{+} / 2, h}^{N,(1)}\left(d_{-f_{+} / 2, h} \alpha\right)=\lambda_{h} d_{-f_{+} / 2, h} \alpha,
$$

which implies, by (2.1.6):

$$
d_{-f_{+} / 2, h} \alpha=h \partial_{x_{n}} \alpha-\frac{1}{2}\left(\partial_{x_{n}} f_{+}\right) \alpha=0 .
$$

Hence:

$$
\alpha\left(x_{n}\right)=C e^{f_{+}\left(x_{n}\right) / 2 h} .
$$

The 0 -form $e^{f_{+}\left(x_{n}\right) / 2 h}$ belongs to $\operatorname{Ker}\left(\Delta_{-f_{+} / 2, h}^{N,(0)}\right)$, so $\lambda_{h}=0$.

(b) The case $n>1$.

First note that any $\omega \in \Lambda^{p} H_{0, \mathbf{n}}^{1}\left(\mathbb{R}_{-}^{n}\right)$ is a sum

$$
\omega=\sum_{\# I=p-1} \alpha_{I}(x) d x^{\prime I} \wedge d x_{n}+\sum_{\# J=p} \beta_{J}(x) d x^{\prime J}=: \alpha \wedge d x_{n}+\beta,
$$

with $\alpha_{I}, \beta_{J} \in H^{1}\left(\mathbb{R}_{-}^{n}\right), \alpha_{I}\left(x^{\prime}, 0\right)=0$, while $d x^{\prime I}=d x_{i_{1}}^{\prime} \wedge \cdots \wedge d x_{i_{\# I}}^{\prime}$, $I=\left\{i_{1}<\ldots<i_{\# I}\right\} \subset\{1, \ldots, n-1\}$ and $J=\left\{j_{1}<\ldots<j_{\# J}\right\} \subset$ $\{1, \ldots, n-1\}$.

If in addition $\omega \in \Lambda^{p} H^{2}\left(\mathbb{R}_{-}^{n}\right)$, the condition $\mathbf{n} d \omega=0$ reads, with the metric $g, \partial_{x_{n}} \beta_{J}\left(x^{\prime}, 0\right)=0$.

Secondly, we remind the reader that with the product metric $g$ the Riemannian connection, the Riemann tensor and therefore the Hodge Laplacian, owing to the Weitzenböck formula, split like direct sums:

$$
\begin{aligned}
\nabla_{X} Y & =\nabla_{X_{n}}^{n} Y_{n}+\nabla_{X^{\prime}}^{\prime} Y^{\prime}, \\
\operatorname{Riem}(x, y, z, t) & =\operatorname{Riem}^{n}\left(x_{n}, y_{n}, z_{n}, t_{n}\right)+\operatorname{Riem}^{\prime}\left(x^{\prime}, y^{\prime}, z^{\prime}, t^{\prime}\right), \\
R_{(4)} & =\sum_{i j k l} \operatorname{Riem}_{i j k l}\left(d x_{i} \wedge\right) \circ \mathbf{i}_{\nabla x_{j}} \circ\left(d x_{k} \wedge\right) \circ \mathbf{i}_{\nabla x_{\ell}}=R_{(4)}^{n}+R_{(4)}^{\prime}, \\
\left(d+d^{*}\right)^{2} & =\left(d_{x_{n}}+d_{x_{n}}^{*}\right)^{2}+\left(d_{x^{\prime}}+d_{x^{\prime}}^{*}\right)^{2} .
\end{aligned}
$$

We refer the reader to [GHL] (p. 110 and p. 70) for details and more general statements. 
Thirdly, the decomposition $f(x)=-\frac{1}{2} f_{+}\left(x_{n}\right)+\frac{1}{2} f_{-}\left(x^{\prime}\right)$ with the product metric $g$ gives

$$
\begin{aligned}
|\nabla f|^{2} & =\left|\nabla_{x_{n}} f\right|^{2}+\left|\nabla_{x^{\prime}} f\right|^{2} \\
\mathcal{L}_{\nabla f}+\mathcal{L}_{\nabla f}^{*} & =-\frac{1}{2}\left(\mathcal{L}_{\nabla f_{+}}+\mathcal{L}_{\nabla f_{+}}^{*}\right)+\frac{1}{2}\left(\mathcal{L}_{\nabla f_{-}}+\mathcal{L}_{\nabla f_{-}}^{*}\right) .
\end{aligned}
$$

For $\omega=\alpha \wedge d x_{n}+\beta \in D\left(\Delta_{f, h}^{N}\right)$ (with the product metric $g$ ), we have

$$
\mathcal{D}_{g, f, h}(\omega)=\left\langle\omega \mid \Delta_{f, h} \omega\right\rangle=\left\langle\omega \mid \Delta_{-f_{+} / 2, h}^{n} \omega\right\rangle+\left\langle\omega \mid \Delta_{f_{-} / 2, h}^{\prime} \omega\right\rangle .
$$

Since the two operators $\Delta_{-f_{+} / 2, h}^{n}$ (acting only in the variable $x_{n}$ ) and $\Delta_{f_{-} / 2, h}^{\prime}$ (acting only in the variable $x^{\prime}$ ) preserve the partial degree in $d x_{n}$, we get

$$
\begin{aligned}
\mathcal{D}_{g, f, h}(\omega)=\langle\alpha & \wedge d x_{n}\left|\Delta_{-f_{+} / 2, h}^{n}\left(\alpha \wedge d x_{n}\right)\right\rangle+\left\langle\beta \mid \Delta_{-f_{+} / 2, h}^{n} \beta\right\rangle \\
& +\left\langle\alpha \wedge d x_{n} \mid \Delta_{f_{-} / 2, h}^{\prime}\left(\alpha \wedge d x_{n}\right)\right\rangle+\left\langle\beta \mid \Delta_{f_{-} / 2, h}^{\prime} \beta\right\rangle
\end{aligned}
$$

Hence the variables $\left(x^{\prime}, x_{n}\right)$ can be separated. The equivalence between the norms $\left\|\sum_{J} \gamma_{J}\left(x^{\prime}\right) d x^{\prime}\right\|$ and $\sum_{J}\left\|\gamma_{J}\left(x^{\prime}\right)\right\|$ on $\Lambda^{p} T^{*} \mathbb{R}_{-}^{n}$, where $J=\left\{j_{1}<\ldots<j_{\# J}\right\} \subset\{1, \ldots, n-1\}$, leads to ${ }^{2}$ :

$$
\begin{gathered}
\mathcal{D}_{g, f, h}(\omega) \geq \\
\frac{1}{c} \int_{\mathbb{R}^{n-1}}\left[\sum_{\# I=p-1} \mathcal{D}_{-f_{+} / 2, h}^{n}\left(\alpha_{I}\left(x^{\prime}, .\right) d x_{n}\right)+\sum_{\# J=p} \mathcal{D}_{-f_{+} / 2, h}^{n}\left(\beta_{J}\left(x^{\prime}, .\right)\right)\right] d \lambda\left(x^{\prime}\right) \\
\quad+\int_{-\infty}^{0} \mathcal{D}_{f_{-} / 2, h}^{\prime}\left(\alpha\left(., x_{n}\right)\right)+\mathcal{D}_{f_{-} / 2, h}^{\prime}\left(\beta\left(., x_{n}\right)\right) d x_{n}, \quad(3.2 .17)
\end{gathered}
$$

where we used the notations $\mathcal{D}_{f_{-} / 2, h}^{\prime}$ for the quadratic form of the Witten Laplacian on $\mathbb{R}^{n-1}$ and $\mathcal{D}_{-f_{+} / 2, h}$ for the quadratic form of the 1-dimensional Witten Laplacian on $\mathbb{R}_{-}$with boundary conditions. The measure $d \lambda\left(x^{\prime}\right)$ simply equals $\left(\operatorname{det} G\left(x^{\prime}\right)\right)^{1 / 2} d x^{\prime}$. The absence of $\alpha-\beta$ cross product term is due to (3.2.16).

Again there are two subcases.

(b1) Subcase $\partial_{x_{n}} f_{+}(0)<0$ :

\footnotetext{
${ }^{2}$ In [HeNi1], at this level of the proof, one should read " $\mathcal{D}_{\tilde{g}, f, h}(\omega) \geq \frac{1}{c} \int_{\mathbb{R}^{n-1}} \ldots$ " instead of " $\mathcal{D}_{\tilde{g}, f, h}(\omega)$ equals $\int_{\mathbb{R}^{n-1}} \ldots$ " accordingly to 2 .
} 
The analysis of the one dimensional problem implies the existence of $c_{1}>0$ independent of $x^{\prime}$ such that:

$$
\begin{array}{ll} 
& \mathcal{D}_{-f_{+} / 2, h}^{n}\left(\alpha_{I}\left(x^{\prime}, .\right) d x_{n}\right) \geq c_{1}\left\|\alpha_{I}\left(x^{\prime}, .\right)\right\|^{2} \\
\text { and } & \mathcal{D}_{-f_{+} / 2, h}^{n}\left(\beta_{J}\left(x^{\prime}, .\right)\right) \geq c_{1}\left\|\beta_{J}\left(x^{\prime}, .\right)\right\|^{2} .
\end{array}
$$

Hence there exists $c_{2}>0$ such that:

$$
\forall \omega \in \Lambda^{p} H_{0, \mathbf{n}}^{1}, \quad \mathcal{D}_{g, f, h}(\omega) \geq c_{2}\|\omega\|^{2}
$$

and

$$
\Delta_{f, h}^{N,(p)} \geq c_{2} \operatorname{Id}, \forall p \in\{0, \ldots, n\}
$$

(b2) Subcase $\partial_{x_{n}} f_{+}(0)>0$ :

Then there exists $c_{1}>0$ such that

$$
\begin{array}{r}
\mathcal{D}_{g, f, h}(\omega) \geq \frac{1}{c} \int_{\mathbb{R}^{n-1}} \sum_{\# J=p} \mathcal{D}_{-f_{+} / 2, h}^{n}\left(\beta_{J}\left(x^{\prime}, .\right)\right) d \lambda\left(x^{\prime}\right) \\
\quad+\int_{-\infty}^{0} \mathcal{D}_{f_{-} / 2, h}^{\prime}\left(\beta\left(., x_{n}\right)\right)+c_{1}\|\alpha\|^{2}
\end{array}
$$

If $\omega$ is a $p$-form with $p \neq p_{0}(\operatorname{deg} \beta=\operatorname{deg} \omega)$, the lower bound

$$
\mathcal{D}_{f_{-} / 2, h}^{\prime}(\beta) \geq C_{1}^{-1} h\|\beta\|^{2},
$$

which was given in Proposition 3.2.3, yields:

$$
\mathcal{D}_{g, f, h}(\omega) \geq C^{-1} h\|\omega\|^{2},
$$

while the equality $\mathcal{D}_{g, f, h}(\omega)=0$ implies that $p=p_{0}$ and that $\omega=c\left(e^{f_{+}\left(x_{n}\right) / 2 h}\right) \psi_{p_{0}}^{h}$, where $\psi_{p_{0}}$ belongs to the kernel of the $(n-1)$ dimensional Witten Laplacian associated with the metric

$$
g^{\prime}=\sum_{i, j=1}^{n-1} g_{i, j}\left(x^{\prime}, 0\right) d x_{i} d x_{i} .
$$

We have now all the ingredients to check every statement for the metric $\tilde{g}_{0}$.

We focus on the subcase $\partial_{x_{n}} f_{+}(0)>0$, which covers all possibilities.

Statements i) and iv)

Statement i) is a consequence of iv) together with Persson's Lemma in [Per]. 
It is sufficient to check that, for all $R>0$, there exists $c_{R}>0$, such that, for all $\omega \in \Lambda^{p} H_{0, \mathbf{n}}^{1}\left(\mathbb{R}_{-}^{n}\right)$ supported in $\left\{\min \left(\left|x^{\prime}\right|,\left|x_{n}\right|\right)>R\right\}$, one has

$$
\mathcal{D}_{\tilde{g}_{0}, f, h}(\omega) \geq c_{R}\|\omega\|^{2} .
$$

The inequalities (3.2.11) and (3.2.18), together with the estimate

$$
\mathcal{D}_{f_{-} / 2, h}^{\prime}\left(\beta\left(\cdot, x_{n}\right)\right) \geq c_{R}^{\prime}\left\|\beta\left(\cdot, x_{n}\right)\right\|^{2} \quad \text { if } \operatorname{supp} \omega \subset\left\{\left|x^{\prime}\right|>R\right\},
$$

provided by Proposition 3.2.3-iii), yield the result.

\section{Statements ii) and iii)}

If $p \neq p_{0}$ the inequalities (3.2.11), (3.2.18) and the inequality

$$
\mathcal{D}_{f_{-} / 2, h}^{\prime}\left(\beta\left(., x_{n}\right)\right) \geq C^{-1} h\left\|\beta\left(., x_{n}\right)\right\|^{2},
$$

imply

$$
\mathcal{D}_{\tilde{g}_{0}, f, h}(\omega) \geq c_{0} h\|\omega\|^{2}
$$

and

$$
\Delta_{f, h}^{N,(p)} \geq c_{0} h \mathrm{Id} .
$$

If $p=p_{0}$, by Proposition 3.2.5, the only possibility for $\lambda_{h} \in\left[0, c_{0} h\right)$ to be an eigenvalue of $\Delta_{f, h}^{N,\left(p_{0}\right)}$ is $\lambda_{h}=0$.

Assume indeed $\Delta_{f, h}^{N,\left(p_{0}\right)} u_{h}=\lambda_{h} u_{h}$ with $\lambda_{h} \in\left[0, c_{0} h\right)$ and $\left\|u_{h}\right\|=1$.

By Proposition 3.2.5(3.2.5) and (3.2.19), $d_{f, h}^{\left(p_{0}\right)} u_{h}=d_{f, h}^{\left(p_{0}-1\right), *} u_{h}=0$. Thus:

$$
\lambda_{h}=\left\langle\Delta_{f, h}^{N,\left(p_{0}\right)} u_{h} \mid u_{h}\right\rangle=\mathcal{D}_{\tilde{g}_{0}, f, h}\left(u_{h}\right)=0 .
$$

When the metric is $g$, the corresponding spectral subspace is one dimensional and equals $\mathbb{C}\left(e^{f_{+}\left(x_{n}\right) / 2 h}\right) \psi_{p_{0}}^{h}$.

For the metric $\tilde{g}_{0}$, the equation $\Delta_{\tilde{g}_{0}, f, h}^{N,\left(p_{0}\right)} \omega=0$ with $\|\omega\|=1$ (which implies $\left.\mathcal{D}_{\tilde{g}_{0}, f, h}(\omega)=0\right)$ and the inequality (3.2.11) lead to:

$$
C^{2} h^{6 / 5}\left\|\tilde{\chi}_{1}\left(h^{-2 / 5} x_{n}\right) \omega\right\|^{2} \geq \mathcal{D}_{g, f, h}\left(\tilde{\chi}_{1}\left(h^{-2 / 5} x_{n}\right) \omega\right)+\left\|\tilde{\chi}_{2}\left(h^{-2 / 5} x_{n}\right) \omega\right\|^{2} .
$$

Without the last term, Lemma 2.3.5 implies:

$$
\operatorname{dist}_{L^{2}}\left(\tilde{\chi}_{1}\left(h^{-2 / 5} x_{n}\right) \omega, \mathbb{C}\left(e^{f_{+}\left(x_{n}\right) / 2 h}\right) \psi_{p_{0}}^{h}\right) \leq C h^{1 / 10} .
$$

The upper bound of the last term,

$$
\left\|\tilde{\chi}_{2}\left(h^{-2 / 5} x_{n}\right) \omega\right\|^{2} \leq C^{2} h^{6 / 5}
$$

implies:

$$
\operatorname{dist}_{L^{2}}\left(\omega, \mathbb{C}\left(e^{f_{+}\left(x_{n}\right) / 2 h}\right) \psi_{p_{0}}^{h}\right)=\mathcal{O}\left(h^{1 / 10}\right) \text {. }
$$


It remains to check that $\operatorname{Ker} \Delta_{f, h}^{N,\left(p_{0}\right)}$ is not reduced to $\{0\}$. The statements of Lemma 3.2.9 and Lemma 3.2.10 are symmetric with respect to the choice of the metric. Hence the reverse inequality of (3.2.11) (with exchange of $g$ and $\left.\tilde{g}_{0}\right)$,

$$
\begin{aligned}
\mathcal{D}_{g, f, h}(\omega) \geq C^{-1} \mathcal{D}_{\tilde{g}_{0}, f, h}\left(\tilde{\chi}_{1}\left(h^{-2 / 5} x_{n}\right) \omega\right) & -C h^{6 / 5}\left\|\tilde{\chi}_{1}\left(h^{-2 / 5} x_{n}\right) \omega\right\|^{2} \\
& +C^{-1}\left\|\tilde{\chi}_{2}\left(h^{-2 / 5} x_{n}\right) \omega\right\|^{2},
\end{aligned}
$$

also holds for any $\omega \in \Lambda H_{0, \mathbf{n}}^{1}\left(\mathbb{R}_{-}^{n}\right)$. We apply it with $\omega=\left(e^{f_{+}\left(x_{n}\right) / 2 h}\right) \psi_{p_{0}}^{h}$ and this leads to:

$$
\mathcal{D}_{\tilde{g}_{0}, f, h}\left(\tilde{\chi}_{1}\left(h^{-2 / 5} x_{n}\right) \omega^{h}\right) \leq C h^{6 / 5}\left\|\tilde{\chi}_{1}\left(h^{-2 / 5} x_{n}\right) \omega\right\|^{2} .
$$

The Min-Max principle then says that $\Delta_{f, h}^{N,\left(p_{0}\right)}$ admits an eigenvalue smaller than $C h^{6 / 5}$. It has to be 0 due to the above argument.

\subsection{Proof of Theorem 3.1.5}

We end here the proof of Theorem 3.1.5 by introducing, after a partition of unity, convenient coordinates which allow the comparison with the model half-space problem.

That proof is almost the same as the proof of the corresponding theorem in [HeNi1], but we recall it for completeness.

Proof of Theorem 3.1.5.

Let $\left\{U_{k}, 1 \leq k \leq K\right\}$ denote the union of the critical points of $f$ and $\left.f\right|_{\Omega}$. Consider a partition of unity of $\bar{\Omega}, \sum_{k=1}^{N} \chi_{k}^{2}=1$, such that the $\mathcal{C}_{0}^{\infty}(\bar{\Omega})$ function $\chi_{k}$ identically equals 1 in a neighborhood of $U_{k}$ when $1 \leq k \leq K$. A refinement of this partition of unity will be specified later by the local construction of adapted coordinates.

We recall that the operator $\Delta_{f, h}^{N}$ is the Friedrichs extension associated with the quadratic form:

$$
\mathcal{D}_{g_{0}, f, h}(\omega)=\left\|d_{f, h} \omega\right\|_{\Lambda L^{2}, g_{0}}^{2}+\left\|d_{f, h}^{*, g_{0}} \omega\right\|_{\Lambda L^{2}, g_{0}}^{2},
$$

on $\Lambda H_{0, \mathbf{n}}^{1}(\Omega)$. The IMS localization formula (3.2.6) gives, for any $\omega \in$ $\Lambda H_{0, \mathbf{n}}^{1}$,

$$
\mathcal{D}_{g_{0}, f, h}(\omega)=\sum_{k=1}^{N} \mathcal{D}_{g_{0}, f, h}\left(\chi_{k} \omega\right)-h^{2}\left\|\left|\nabla \chi_{k}\right| \omega\right\|_{\Lambda L^{2}, g_{0}}^{2} .
$$

If supp $\chi_{k}$ does not meet the boundary, the term $\mathcal{D}_{g_{0}, f, h}\left(\chi_{k} \omega\right)$ behaves like in the boundaryless case (see $[\mathrm{HKN}]$ for details): 
- If $k>K$, then we have

$$
\forall \omega \in \Lambda H^{1}, \quad \mathcal{D}_{g_{0}, f, h}\left(\chi_{k} \omega\right) \geq C^{-1}\left\|\chi_{k} \omega\right\|_{\Lambda L^{2}, g_{0}}^{2} .
$$

- If $k \leq K$ and $U_{k}$ is a critical point of $f$ with index $p_{k} \neq p$, then

$$
\forall \omega \in \Lambda H^{1}, \quad \mathcal{D}_{g_{0}, f, h}\left(\chi_{k} \omega\right) \geq C^{-1} h\left\|\chi_{k} \omega\right\|_{\Lambda L^{2}, g_{0}}^{2} .
$$

- If $k \leq K$ and $U_{k}$ is a critical point of $f$ with index $p_{k}=p$, then there exists a fixed 1-dimensional space $F_{k}^{(p)}$ (determined by Hess $f\left(U_{k}\right)$ ) such that,

$$
\forall \omega \in \Lambda H^{1}, \quad \mathcal{D}_{g_{0}, f, h}\left(\chi_{k} \omega\right) \leq C^{-1} h^{6 / 5}\left\|\chi_{k} \omega\right\|_{\Lambda^{p} L^{2}, g_{0}}^{2}
$$

implies

$$
\forall \omega \in \Lambda H^{1}, \quad \operatorname{dist}\left(\chi_{k} \omega, F_{k}^{(p)}\right) \leq C h^{1 / 10}\|\omega\|_{\Lambda^{p} L^{2}, g_{0}} .
$$

Again like in the proof of Proposition 3.2.11-iii), this last statement refers to Lemma 2.3.5 at the level of quadratic forms.

Consider now the case when $\operatorname{supp} \chi_{k} \cap \partial \Omega \neq \emptyset$, with the support of $\chi_{k}$ centered around a point $U_{0} \in \partial \Omega$. There are two cases: $U_{0}$ is a critical point of $\left.f\right|_{\partial \Omega}$ with $\frac{\partial f}{\partial n}\left(U_{0}\right)<0$ which is equivalent to $-\frac{\partial f}{\partial n}\left(U_{0}\right)=\left|\nabla f\left(U_{0}\right)\right|$ or $U_{0}$ is not a critical points of $\left.f\right|_{\partial \Omega}$ with $\frac{\partial f}{\partial n}<0$ which is equivalent to $\left(-\frac{\partial f}{\partial n}\right)\left(U_{0}\right)<\left|\nabla f\left(U_{0}\right)\right|$. Indeed, $U_{0}$ is either a critical point of $\left.f\right|_{\partial \Omega}$ with $\frac{\partial f}{\partial n}\left(U_{0}\right)>0$, i.e. $\frac{\partial f}{\partial n}\left(U_{0}\right)=\left|\nabla f\left(U_{0}\right)\right|$ or $U_{0}$ is not a critical point of $\left.f\right|_{\partial \Omega}$, i.e. $\left|\frac{\partial f}{\partial n}\left(U_{0}\right)\right|<\left|\nabla f\left(U_{0}\right)\right|$.

Case 1) $\left(-\frac{\partial f}{\partial n}\right)\left(U_{0}\right)<\left|\nabla f\left(U_{0}\right)\right|$.

Then the cut-off $\chi_{k}$ is chosen so that, in a neighborhood $\mathcal{V}$ of supp $\chi_{k}$,

$$
\forall x \in \mathcal{V} \cap \partial \Omega, \quad\left(-\frac{\partial f}{\partial n}\right)(x)<(1-\delta)|\nabla f(x)|,
$$

for some $\delta>0$. Locally it is possible to construct a function $\hat{f}$ such that $-\partial_{n} \hat{f}=|\nabla \hat{f}|$ in $\mathcal{V} \cap \partial \Omega$ and $|\nabla \hat{f}|=|\nabla f|$ in $\mathcal{V}$. By setting $\tilde{\omega}=\chi_{k} \omega$ for $\omega \in \Lambda H_{0, \mathbf{n}}^{1}$, the Green formula (2.2.9) and the inequality $\mathcal{D}_{g_{0}, \hat{f}, h}(\tilde{\omega}) \geq 0$ imply $\left(\mathcal{L}_{\nabla f}+\mathcal{L}_{\nabla f}^{*}\right.$ being a tensor $)$

$$
\begin{aligned}
& -h \int_{\partial \Omega}\langle\tilde{\omega} \mid \tilde{\omega}\rangle_{\Lambda^{p} T_{\sigma}^{*} \Omega}\left(\frac{\partial f}{\partial n}\right)(\sigma) d \sigma \leq-(1-\delta) h \int_{\partial \Omega}\langle\tilde{\omega} \mid \tilde{\omega}\rangle_{\Lambda^{p} T_{\sigma}^{*} \Omega}\left(\frac{\partial \hat{f}}{\partial n}\right)(\sigma) d \sigma \\
& \leq(1-\delta)\left[h^{2}\|d \tilde{\omega}\|_{\Lambda^{p+1} L^{2}}^{2}+h^{2}\left\|d^{*} \tilde{\omega}\right\|_{\Lambda^{p-1} L^{2}}^{2}+\||\nabla f| \tilde{\omega}\|_{\Lambda^{p} L^{2}}^{2}+C_{1} h\|\tilde{\omega}\|_{\Lambda^{p} L^{2}}^{2}\right] .
\end{aligned}
$$


- If $k>K$,

$$
\begin{aligned}
\forall \omega \in \Lambda H_{0, \mathbf{n}}^{1}, \quad \mathcal{D}_{g_{0}, f, h}\left(\chi_{k} \omega\right)=\mathcal{D}_{g_{0}, f, h}(\tilde{\omega}) & \geq \frac{\delta}{2}\||\nabla f| \tilde{\omega}\|_{\Lambda^{p} L^{2}}^{2} \\
& \geq C_{\mathcal{V}}^{-1}\left\|\chi_{k} \omega\right\|_{\Lambda^{p} L^{2}}^{2} .
\end{aligned}
$$

Case 2) $-\frac{\partial f}{\partial n}\left(U_{0}\right)=\left|\nabla f\left(U_{0}\right)\right|$.

In this case we will conclude by applying Proposition 3.2.11. We recall that $U_{0} \in \partial \Omega$ is a critical point of $\left.f\right|_{\partial \Omega}$ with $\frac{\partial f}{\partial n}\left(U_{0}\right)<0$ and with index $p_{0}$. Around $U_{0}$, we introduce adapted local coordinates, denoted by $\bar{x}=\left(\bar{x}^{\prime}, \bar{x}_{n}\right)$. This coordinate system is provided by Lemma 3.3.1 below, applied with $f_{1}=f$ and $\alpha=\left.f\right|_{\partial \Omega \cap \mathcal{V}_{0}}$. Then the function $\Phi_{+}$of Lemma 3.3.1 is nothing but $f$ and has the form $f(\bar{x})=-\bar{x}_{n}+\frac{1}{2} f_{-}\left(\bar{x}^{\prime}\right)$. Moreover, $\bar{\Omega}$ corresponds locally to $\left\{\bar{x}_{n} \leq 0\right\}$.

In order to apply Proposition 3.2.11, it remains to check that the function $f$ can be extended to $\mathbb{R}_{-}^{n}$, so that it satisfies Assumption 3.2.4 where $U_{0}$ is a critical point of $\left.f\right|_{\partial \Omega}$.

We recall that we have not specified the choice of $\bar{x}^{\prime}$ in the boundary. The function $\left.f\right|_{\partial \Omega \cap \mathcal{V}_{0}}$ being a Morse function, we can choose in a small neighborhood $\mathcal{V}_{0}^{\prime} \subset \partial \Omega$ of $U_{0}=(0, \ldots, 0)$ Morse coordinates $\bar{x}^{\prime}=\left(\bar{x}_{1}, \ldots, \bar{x}_{n-1}\right)$ for $f_{-}$which are normal at $U_{0}$ for the metric $\sum_{i, j<n} g_{i j}\left(\bar{x}^{\prime}, 0\right) d \bar{x}_{i} d \bar{x}_{j}$. With these coordinates, $f$ has the form, in a small neighborhood $\mathcal{V}_{0}^{\prime \prime}$ of 0 :

$$
f(\bar{x})=-\bar{x}_{n}+\sum_{j=1}^{n-1} \lambda_{j} \bar{x}_{j}^{2}+f\left(U_{0}\right) .
$$

We choose $\chi_{k}$ such that $\operatorname{supp} \chi_{k} \subset \mathcal{V}_{0}^{\prime \prime}$.

Choosing a cut-off $\chi^{n-1} \in \mathcal{C}_{0}^{\infty}\left(\mathbb{R}^{n-1}\right), \chi^{n-1}=1$ near $\operatorname{supp} \chi_{k} \cap \partial \Omega, f$ is extended to $\mathbb{R}_{-}^{n}$ by:

$$
\tilde{f}(\bar{x})=-\bar{x}_{n}+\left[\chi^{n-1}\left(\bar{x}^{\prime}\right)+\frac{1-\chi^{n-1}\left(\bar{x}^{\prime}\right)}{\left|\bar{x}^{\prime}\right|}\right]\left[\sum_{j=1}^{n-1} \lambda_{j} \bar{x}_{j}^{2}\right]+f\left(U_{0}\right) .
$$

Moreover, choosing another cut-off $\chi^{n} \in \mathcal{C}_{0}^{\infty}\left(\overline{\mathbb{R}_{-}^{n}}\right), \chi^{n}=1$ near supp $\chi_{k}$, we extend $g_{0}$ to $\mathbb{R}_{-}^{n}$ by:

$$
\tilde{g}=\chi^{n} g_{0}+\left(1-\chi^{n}\right) g_{e},
$$

where $g_{e}$ is the Euclidian metric on $\mathbb{R}_{-}^{n}$.

With these coordinates, the quantity $\mathcal{D}_{\tilde{g}, \tilde{f}, h}\left(\chi_{k} \omega\right)=\mathcal{D}_{g_{0}, f, h}\left(\chi_{k} \omega\right)$ attains the form discussed in Proposition 3.2.11.

We can now discuss the lower bound of $\mathcal{D}_{\tilde{g}, \tilde{f}, h}\left(\chi_{k} \omega\right)$, depending on the localization by the cut-off $\chi_{k}$, such that $\operatorname{supp} \chi_{k} \cap \partial \Omega \neq \emptyset$. 
- If $k \leq K$, the origin of the coordinate system is $U_{0}=U_{k}$. If $U_{k}$ is not a critical point of $\left.f\right|_{\partial \Omega}$ with index $p_{k}=p$ and $\frac{\partial f}{\partial n}\left(U_{k}\right)<0$, then

$$
\forall \omega \in \Lambda^{p} H_{0, \mathbf{n}}^{1}, \quad \mathcal{D}_{\tilde{g}, \tilde{f}, h}\left(\chi_{k} \omega\right) \geq C^{-1} h\left\|\chi_{k} \omega\right\|_{\Lambda L^{2}, g}^{2} .
$$

- If $k \leq K$ and $U_{k}$ is a critical point of $\left.f\right|_{\partial \Omega}$ with index $p_{k}=p$ and $\frac{\partial f}{\partial n}\left(U_{k}\right)<0$, then according to Proposition 3.2.11-iii) there exists a fixed 1-dimensional space $F_{k}^{(p)}$ such that the inequality,

$$
\forall \omega \in \Lambda^{p} H_{0, \mathbf{n}}^{1}, \quad \mathcal{D}_{\tilde{g}, \tilde{f}, h}\left(\chi_{k} \omega\right) \leq C^{-1} h^{6 / 5}\left\|\chi_{k} \omega\right\|_{\Lambda^{p} L^{2}, g}^{2}
$$

implies:

$$
\operatorname{dist}\left(\chi_{k} \omega, F_{k}^{(p)}\right) \leq C h^{1 / 10}\left\|\chi_{k} \omega\right\|_{\Lambda^{p} L^{2}, g} .
$$

We now introduce the set $A_{p}$ of indices $k, 1 \leq k \leq K$, such that

- either $U_{k}$ is a critical point of $f$ with index $p$,

- or $U_{k}$ is a critical point of $\left.f\right|_{\partial \Omega}$ with index $p$ such that $\frac{\partial f}{\partial n}\left(U_{k}\right)<0$.

For $\omega \in \Lambda^{p} H_{0, \mathbf{n}}^{1}(\Omega)$ with $\|\omega\|_{\Lambda^{p} L^{2}, g}=1$, we get

$$
\left(\mathcal{D}_{g_{0}, f, h}(\omega) \leq C^{-1} h^{6 / 5}\right) \Rightarrow\left(\operatorname{dist}\left(\omega, \sum_{k \in A_{p}} F_{k}^{(p)}\right) \leq C h^{1 / 10}\right)
$$

Hence the dimension of the spectral subspace,

$$
F^{(p)}=\operatorname{Ran}_{\left[0, h^{3 / 2}\right)}\left(\Delta_{f, h}^{N,(p)}\right) \subset \operatorname{Ran}_{\left[0, c h^{6 / 5}\right)}\left(\Delta_{f, h}^{N,(p)}\right),
$$

is at most $\# A_{p}=m_{p}^{\bar{\Omega}}$.

We next verify that $\operatorname{dim} F^{(p)} \geq \# A_{p}=m_{p}^{\bar{\Omega}}$. According to the Min-Max principle, it suffices to find an orthonormal set of $p$-forms $\omega_{k}^{h} \in \Lambda^{p} H_{0, \mathbf{n}}^{1}(\Omega)$, $k \in A_{p}$, such that

$$
\mathcal{D}_{g_{0}, f, h}\left(\omega_{k}^{h}\right)=o\left(h^{3 / 2}\right) .
$$

Indeed it is enough to take a truncated element of the kernel of the local model for $\Delta_{f, h}^{N,(p)}$ around $U_{k}, k \in A_{p}$. We give the details for the case $U_{k} \in \partial \Omega$.

Take two cut-off $\chi_{1, k} \in \mathcal{C}_{0}^{\infty}\left(\overline{\mathbb{R}_{-}^{n}}\right), \chi_{1, k}=1$ near $0\left(\right.$ with $\operatorname{supp} \chi_{1, k} \subset \operatorname{supp} \chi_{k}$ ) and $\chi_{2, k}$ such that $\chi_{1, k}^{2}+\chi_{2, k}^{2}=1$. With the same coordinate system as above, we write on $\mathbb{R}_{-}^{n}$, using the IMS localization formula (3.2.6) and Proposition 3.2.11-iv),

$$
\mathcal{D}_{\tilde{g}_{k}, \tilde{f}_{k}, h}(\omega) \geq \mathcal{D}_{\tilde{g}_{k}, \tilde{f}_{k}, h}\left(\chi_{1, k} \omega\right)+C^{-1}\left\|\chi_{2, k} \omega\right\|^{2}-C h^{2} \sum_{i=1,2}\left\|\left|\nabla \chi_{i, k}\right| \omega\right\|^{2},
$$


where $\tilde{g}_{k}$ and $\tilde{f}_{k}$ are defined on $\mathbb{R}_{-}^{n}$ according to the previous construction and coincide with $g_{0}$ and $f$ in a neighborhood of supp $\chi_{k}$. According to Proposition 3.2.11, there exists $\eta_{k}^{h} \in \Lambda^{p} H_{0, \mathbf{n}}^{1}\left(\mathbb{R}_{-}^{n}\right)$ in the domain of the associated Witten Laplacian, such that $\mathcal{D}_{\tilde{g}_{k}, \tilde{f}_{k}, h}\left(\eta_{k}^{h}\right)=0$. By taking $\omega_{k}^{h}=\left\|\chi_{1, k} \eta_{k}^{h}\right\|^{-1} \chi_{1, k} \eta_{k}^{h}$, we obtain the existence of $h_{0}>0, C^{\prime}$ and $C^{\prime \prime}$ such that, for $h \in\left(0, h_{0}\right]$ :

and, consequently,

$$
\left\|\chi_{2, k} \eta_{k}^{h}\right\|^{2} \leq C^{\prime} h^{2}\left\|\eta_{k}^{h}\right\|^{2}
$$

$$
\mathcal{D}_{g_{0}, f, h}\left(\omega_{k}^{h}\right) \leq C^{\prime} h^{2} \frac{\left\|\eta_{k}^{h}\right\|^{2}}{\left\|\chi_{1, k} \eta_{k}^{h}\right\|^{2}} \leq C^{\prime \prime} h^{2}
$$

The next lemma, which provides in different situations the suitable coordinate systems, simply makes use of the standard solution to Hamilton-Jacobi equations in the non characteristic case. It is proved in [Lep1].

Lemma 3.3.1. 1) Let be $f_{1} \in \mathcal{C}^{\infty}(\bar{\Omega}, \mathbb{R})$ and $U_{0} \in \partial \Omega$ a critical point of $\left.f_{1}\right|_{\partial \Omega}$ with $\frac{\partial f_{1}}{\partial n}\left(U_{0}\right) \neq 0$.

Assume furthermore $\alpha \in \mathcal{C}^{\infty}(\partial \Omega, \mathbb{R})$ be a local solution to $\left|\nabla_{T} \alpha\right|^{2}=\left|\nabla_{T} f_{1}\right|^{2}$ around $U_{0}$.

Then there exists a neighborhood $\mathcal{V}_{0}$ of $U_{0}$ in $\bar{\Omega}$ such that the eikonal equation:

$$
\left|\nabla \Phi_{ \pm}\right|^{2}=\left|\nabla f_{1}\right|^{2}
$$

(on the boundary, it means $\left|\partial_{n} \Phi_{ \pm}\right|^{2}+\left|\nabla_{T} \Phi_{ \pm}\right|^{2}=\left|\partial_{n} f_{1}\right|^{2}+\left|\nabla_{T} f_{1}\right|^{2}$ ) with the boundary conditions

$$
\left.\Phi_{ \pm}\right|_{\partial \Omega \cap \mathcal{V}_{0}}=\alpha \quad,\left.\quad \partial_{n} \Phi_{ \pm}\right|_{\partial \Omega \cap \mathcal{V}_{0}}= \pm\left.\frac{\partial f_{1}}{\partial n}\right|_{\partial \Omega \cap \mathcal{V}_{0}}
$$

admits a unique local smooth real-valued solution.

2) There exists local coordinates $\left(x_{1}, \ldots, x_{n}\right)=\left(x^{\prime}, x_{n}\right)$ in a neighborhood of $U_{0}$ in $\bar{\Omega}$ with $\left(x^{\prime}, x_{n}\right)\left(U_{0}\right)=0$ where the function $\Phi_{ \pm}$and the metric $g_{0}$ have the form:

$$
\Phi_{ \pm}=\mp x_{n}+\alpha\left(x^{\prime}\right) \quad \text { and } \quad g_{0}=g_{n n}(x) d x_{n}^{2}+\sum_{i, j=1}^{n-1} g_{i j}(x) d x_{i} d x_{j}
$$

Moreover, the boundary $\partial \Omega$ is locally defined by $\left\{x_{n}=0\right\}$ and $\Omega$ corresponds to $\left.\left\{\operatorname{sgn}\left(\frac{\partial f_{1}}{\partial n}\left(U_{0}\right)\right)\right) x_{n}>0\right\}$. 
Remark 3.3.2. Lemma 3.3.1 will be used with various functions $f_{1}$ and $\alpha$ and will provide several coordinate systems:

- We have already introduced the coordinate system $\bar{x}=\left(\bar{x}, \bar{x}_{n}\right)$ associated with $f_{1}=f$ and $\alpha=\left.f\right|_{\partial \Omega}$.

- The coordinate system denoted simply by $x=\left(x^{\prime}, x_{n}\right)$ will be associated with $f_{1}=f$ and $\alpha=\varphi$, where $\varphi$ is the Agmon distance along the boundary. This system will be used to give the simple form $\Phi=\Phi_{+}=$ $-x_{n}+\varphi\left(x^{\prime}\right)$ to the Agmon distance $\Phi$, solving $|\nabla \Phi|^{2}=|\nabla f|^{2}$ with the boundary condition $\partial_{n} \Phi=\partial_{n} f$. Agmon distances are specified in Section 4 below.

- Finally the coordinate system $\tilde{x}=\left(\tilde{x}^{\prime}, \tilde{x}_{n}\right)$ will be associated with $f_{1}=$ $(f+\Phi)$ and $\alpha=\left.f\right|_{\partial \Omega}+\varphi$ and will be used in the final application of the Laplace method.

\section{Accurate WKB analysis near the boundary for $\Delta_{f, h}^{(1)}$}

\subsection{Introduction}

We work here under Assumption 3.1.1. Like in [HeNi1], we have shown that for $0 \leq p<n$, some quasimodes of $\Delta_{f, h}^{N,(p)}$ being near the spectral subspace in $1_{\left[0, h^{\frac{3}{2}}\right)}\left(\Delta_{f, h}^{N,(p)}\right)$ are localized near the boundary $\partial \Omega$ and more precisely near critical points of $\left.f\right|_{\partial \Omega}$ with index $p$ such that $\frac{\partial f}{\partial n}<0$. In the boundaryless case $([\mathrm{HKN}])$ and in the case with tangential Dirichlet boundary conditions ([HeNi1]), the WKB analysis done in $[\mathrm{HeSj} 4]$ and in [HeNi1] says that the small eigenvalues are of order $\mathcal{O}\left(e^{-C / h}\right)$ and provides an accurate approximate basis of $\operatorname{Ran} 1_{\left[0, h^{3 / 2}\right)}\left(\Delta_{f, h}^{(p)}\right)$.

In order to get a similar result, we need an accurate WKB analysis at the boundary, and like in [HeNi1], we restrict our attention on the case $p=1$ because our motivation is to analyze the Witten Laplacian on 0 -forms.

For an accurate comparison between eigenvectors and WKB quasimodes near a critical point $U_{1}$ of $\left.f\right|_{\partial \Omega}$ with index 1 and $\frac{\partial f}{\partial n}\left(U_{1}\right)<0$, we introduce another self-adjoint realization of $\Delta_{f, h}^{(1)}$ in a neighborhood $\Omega_{U_{1}, \rho}$ with mixed boundary conditions: Neumann boundary conditions on $\partial \Omega_{U_{1}, \rho} \cap \partial \Omega$ and full Dirichlet boundary conditions on $\partial \Omega_{U_{1}, \rho} \backslash \partial \Omega$.

\subsection{Local WKB construction}

Take $U_{1}$ a critical point of $\left.f\right|_{\partial \Omega}$ with index 1 such that $\frac{\partial f}{\partial n}\left(U_{1}\right)<0$. According to [Lep1], there exists a local coordinate system $\left(\underline{x}_{1}, \ldots, \underline{x}_{n}\right)=\left(\underline{x}^{\prime}, \underline{x}_{n}\right)$ which satisfies the next properties: 
i) $d \underline{x}_{1}, \ldots, d \underline{x}_{n}$ is an orthonormal basis of $T_{U_{1}}^{*}(\bar{\Omega})$ positively oriented.

ii) The boundary $\partial \Omega$ corresponds locally to $\underline{x}_{n}=0$ and the interior $\Omega$ to $\underline{x}_{n}<0$.

iii) $\left.\frac{\partial}{\partial \underline{x}_{n}}\right|_{\partial \Omega}=\vec{n}$, the outgoing normal at the boundary. Moreover, $\frac{\partial}{\partial \underline{x}_{n}}$ is unitary and normal to $\left\{\underline{x}_{n}=\right.$ Constant $\}$.

Moreover, the choice of the coordinates $\left(\underline{x}_{1}, \ldots, \underline{x}_{n-1}\right)$ (centered at $U_{1}$ such that $d \underline{x}_{1}, \ldots, d \underline{x}_{n}$ is an orthonormal basis of $\left.T_{U_{1}}^{*}(\bar{\Omega})\right)$ in the boundary is arbitrary.

Let $\varphi$ be the Agmon distance to $U_{1}$ on the boundary (i.e. associated with the metric $\left.\left|\nabla_{\underline{x}^{\prime}} f\left(\underline{x}^{\prime}, 0\right)\right| d \underline{x}^{\prime 2}\right)$. Recall that $\varphi$ satisfies

$$
\left|\nabla_{T} f\right|^{2}=|\nabla \varphi|^{2}
$$

on the boundary and that $\varphi$ is smooth near $U_{1}$ (see [HeSj1]). Apply now the first point of Lemma 3.3.1 with $f_{1}=f$ and $\alpha=\varphi$ and denote by $\Phi$ the function $\Phi_{+}$of the lemma ( $\Phi$ is the Agmon distance to $U_{1}$, i.e. associated with the metric $\left.\left|\nabla_{\underline{x}} f(\underline{x})\right| d \underline{x}^{2}\right)$. Hence the next equalities are locally satisfied:

$$
\begin{aligned}
\left|\partial_{n} \Phi\right|^{2}+\left|\nabla_{T} \Phi\right|^{2} & =|\nabla \Phi|^{2}=|\nabla f|^{2}, \\
\left.\Phi\right|_{\partial \Omega} & =\varphi, \\
\left.\partial_{n} \Phi\right|_{\partial \Omega} & =\left.\frac{\partial f}{\partial n}\right|_{\partial \Omega} .
\end{aligned}
$$

According to $[\mathrm{HeSj} 4]$ pp. 279-280, there exist Morse coordinates $\left(v_{1}, \ldots, v_{n-1}\right)$ for $\left.f\right|_{\Omega}$ centered at $U_{1}$ and such that $d v_{1}\left(U_{1}\right), \ldots, d v_{n-1}\left(U_{1}\right), \vec{n}_{U_{1}}^{*}$ is orthonormal and positively oriented. With these coordinates

$$
\begin{aligned}
f(v, 0) & =\frac{\lambda_{1}}{2} v_{1}^{2}+\cdots+\frac{\lambda_{n-1}}{2} v_{n-1}^{2}+f\left(U_{1}\right) \\
& \text { and } \\
\varphi(v) & =\frac{\left|\lambda_{1}\right|}{2} v_{1}^{2}+\cdots+\frac{\left|\lambda_{n-1}\right|}{2} v_{n-1}^{2},
\end{aligned}
$$

with $\lambda_{1}<0$.

Moreover, $\left(x_{1}, \ldots, x_{n-1}\right)$ can be chosen equal to $\left(v_{1}, \ldots, v_{n-1}\right)$ in the boundary. Hence, the theorem of [Lep1] given in the Neumann case implies the next proposition:

Proposition 4.2.1. Consider around $U_{1}$ the above system of coordinates $\underline{x}=\left(\underline{x}^{\prime}, \underline{x}_{n}\right)$ which satisfies $(4.2 .1)(4.2 .2)$ with $\lambda_{1}<0$. There exists locally, in a neighborhood of $\underline{x}=0, a \mathcal{C}^{\infty}$ solution $u_{1}^{w k b}$ to

$$
\begin{aligned}
\Delta_{f, h}^{(1)} u_{1}^{w k b} & =e^{-\frac{\Phi}{h}} \mathcal{O}\left(h^{\infty}\right) \\
\mathbf{n} u_{1}^{w k b} & =0 \text { on } \partial \Omega \\
\mathbf{n} d_{f, h} u_{1}^{w k b} & =0 \text { on } \partial \Omega,
\end{aligned}
$$


where $u_{1}^{w k b}$ has the form:

$$
\begin{aligned}
u_{1}^{w k b} & =a(\underline{x}, h) e^{-\frac{\Phi}{h}}, \\
\text { with } \quad a(\underline{x}, h) & \sim \sum_{k} a^{k}(\underline{x}) h^{k} \text { and } a^{0}(0)=d \underline{x}_{1} .
\end{aligned}
$$

\subsection{Another local Neumann realization of $\Delta_{f, h}^{(1)}$}

Let $U_{1}$ be a critical point of $\left.f\right|_{\partial \Omega}$ with index 1 and $\frac{\partial f}{\partial n}\left(U_{1}\right)<0$ and let us introduce a new system of local coordinates.

We apply Lemma 3.3.1 with $f_{1}=f$ and $\alpha=\varphi$, the Agmon distance to $U_{1}$ on the boundary. The function $\Phi_{+}$of the lemma is then $\Phi$, the Agmon distance to $U_{1}$ and we have the existence of local coordinates $\left(x^{\prime}, x_{n}\right)$ around $U_{1}$ where $\Phi$ and the metric $g_{0}$ have the form:

$$
\Phi=-x_{n}+\varphi\left(x^{\prime}\right) \quad \text { and } \quad g_{0}=g_{n n}(x) d x_{n}^{2}+\sum_{i, j=1}^{n-1} g_{i j}(x) d x_{i} d x_{j} .
$$

Moreover, the boundary $\partial \Omega$ is locally defined by $\left\{x_{n}=0\right\}$ and $\Omega$ corresponds to $\left\{x_{n}<0\right\}$.

We work now with the local coordinate system defined above and $x \mapsto|x|$ is the Euclidean norm in these coordinates.

As in [HeNi1], we consider the domain, for $\rho>0$,

$$
\Omega_{U_{1}, \rho}=\left\{|x-(0,1)|^{2}<\rho^{2}+1, x_{n}<0\right\},
$$

which has the shape of a thin lens stuck on $\partial \Omega$ with radius $\rho$ and thickness $\mathcal{O}\left(\rho^{2}\right)$. Its boundary splits into

and

$$
\Gamma_{D}:=\partial \Omega_{U_{1}, \rho} \cap \Omega=\left\{|x-(0,1)|^{2}=\rho^{2}+1, x_{n} \leq 0\right\}
$$

$$
\Gamma_{N D}:=\partial \Omega_{U_{1}, \rho} \cap \partial \Omega=\left\{\left|x^{\prime}\right|<\rho, x_{n}=0\right\} .
$$

On this domain, we introduce the functional space

$$
\Lambda^{1} H_{0 ; 0, \mathbf{n}}^{1}\left(\Omega_{U_{1}, \rho}\right)=\left\{u \in \Lambda^{1} H^{1}\left(\Omega_{U_{1}, \rho}\right) ;\left.\mathbf{n} u\right|_{\Gamma_{N D}}=0,\left.\quad u\right|_{\Gamma_{D}}=0\right\} .
$$

The Friedrichs extension associated with the quadratic form:

$$
\Lambda^{1} H_{0 ; 0, \mathbf{n}}^{1}\left(\Omega_{U_{1}, \rho}\right) \ni \omega \mapsto \mathcal{D}_{g, f, h}^{N}(\omega)=\left\|d_{f, h} \omega\right\|^{2}+\left\|d_{f, h}^{*} \omega\right\|^{2},
$$

is denoted by $\Delta_{f, h}^{N, D,(1)}$. The domain of $\Delta_{f, h}^{N, D,(1)}$ is contained in $\Lambda^{1} H^{2}\left(\Omega_{U_{1}}, \rho^{\prime}\right)$ for any $0<\rho^{\prime}<\rho$.

An element $\omega \in D\left(\Delta_{f, h}^{N, D,(1)}\right)$ satisfies indeed:

$$
\left\langle\Delta_{f, h}^{N, D,(1)} \omega \mid \eta\right\rangle=\left\langle d_{f, h} \omega \mid d_{f, h} \eta\right\rangle+\left\langle d_{f, h}^{*} \omega \mid d_{f, h}^{*} \eta\right\rangle=: \mathcal{D}_{g, f, h}^{N}(\omega, \eta),
$$


for all $\eta \in \Lambda^{1} H_{0 ; 0, \mathbf{n}}^{1}$. By testing with $\eta \in \mathcal{C}_{0}^{\infty}\left(\Omega_{U_{1}, \rho}\right)$, this gives $\Delta_{f, h} \omega \in$ $\Lambda^{1} L^{2}\left(\Omega_{U_{1}, \rho}\right)$ and therefore $\omega$ admits a second trace on $\Gamma_{N D}$. By testing with any $\eta \in \mathcal{C}_{0 ; 0, \mathbf{n}}^{\infty}\left(\Omega_{U_{0, \rho}}\right)$, we get:

$$
\left.\mathbf{n} d_{f, h} \omega\right|_{\Gamma_{N D}}=0 .
$$

Along $\Gamma_{N D}, \omega$ solves an elliptic boundary value problem $\Delta_{f, h}^{(1)} \omega \in \Lambda^{1} L^{2}$, $\mathbf{n} \omega=0, \mathbf{n} d_{f, h} \omega=0$, which provides the $H^{2}$ regularity in $\Omega_{U_{0, \rho^{\prime}}}$ for any $\rho^{\prime}<\rho$.

We now prove the

\section{Proposition 4.3.1.}

For $\rho>0$ small enough, there exist $h_{\rho}>0$ and $C_{\rho}>0$, such that the selfadjoint operator $\Delta_{f, h}^{N, D,(1)}$ satisfies the following properties:

a) For $h \in\left(0, h_{\rho}\right]$, the spectral projection $1_{\left[0, h^{3 / 2}\right)}\left(\Delta_{f, h}^{N, D,(1)}\right)$ has rank 1 .

b) Any family of $L^{2}$-normalized eigenvectors $\left(u^{h}\right)_{h \in\left(0, h_{\rho}\right]}$ of $\Delta_{f, h}^{N, D,(1)}$ such that the corresponding eigenvalue $E(h)$ is $\mathcal{O}(h)$, satisfies

$$
\begin{aligned}
& \forall \rho^{\prime}<\rho, \forall \alpha \in \mathbb{N}^{n}, \exists N_{\alpha} \in \mathbb{N}, \exists C_{\alpha, \rho^{\prime}}>0 \text { such that, } \forall x \in \Omega_{U_{1}, \rho^{\prime}}, \\
& \left|\partial_{x}^{\alpha} u^{h}(x)\right| \leq C_{\alpha, \rho^{\prime}} h^{-N_{\alpha}} \exp \left(-\frac{\Phi(x)}{h}\right) .
\end{aligned}
$$

c) There exists $\varepsilon_{\rho}>0$ such that the first eigenvalue $E_{1}(h)$ of $\Delta_{f, h}^{N, D,(1)}$ satisfies

$$
E_{1}(h)=\mathcal{O}\left(e^{-\varepsilon_{\rho} / h}\right) .
$$

d) If $u_{1}^{h}$ denotes the eigenvector of $\Delta_{f, h}^{N, D,(1)}$ associated with eigenvalue $E_{1}(h)$ and normalized by the condition $\mathbf{t} u_{1}^{h}(0)=\mathbf{t} u_{1}^{w k b}(0)$, then

$$
\begin{aligned}
& \forall \rho^{\prime}<\rho, \forall \alpha \in \mathbb{N}^{n}, \forall N \in \mathbb{N}, \exists C_{N, \alpha, \rho^{\prime}}>0 \text { such that, } \forall x \in \Omega_{U_{1}, \rho^{\prime}}, \\
& \left|\partial_{x}^{\alpha}\left(u_{1}^{h}-u_{1}^{w k b}\right)(x)\right| \leq C_{N, \alpha, \rho^{\prime}} h^{N} \exp \left(-\frac{\Phi(x)}{h}\right) .
\end{aligned}
$$

Once this is proved, one easily gets rough exponentially small upper bounds for the $m_{\ell}^{\bar{\Omega}}$ first eigenvalues of $\Delta_{f, h}^{N,(\ell)}(\ell \in\{0,1\})$ on $\Omega$, by constructing quasimodes suitably localized near each of the critical points.

The next subsections are devoted to the proof of Proposition 4.3.1. A fondamental ingredient for the proof is a variant of the integration by parts formula of Lemma 2.2.3.

\section{Lemma 4.3.2.}

Let $\rho>0$ and let $\psi$ be a real-valued Lipschitz function on $\overline{\Omega_{U_{1}, \rho}}$. The relation

$$
\begin{aligned}
\operatorname{Re} \mathcal{D}_{g, f, h}^{N}\left(\omega, e^{2 \frac{\psi}{h}} \omega\right)= & h^{2}\left\|d e^{\frac{\psi}{h} \omega}\right\|_{\Lambda^{2} L^{2}}^{2}+h^{2}\left\|d^{*} e^{\frac{\psi}{h} \omega}\right\|_{\Lambda^{0} L^{2}}^{2} \\
+\left\langle\left(|\nabla f|^{2}-\right.\right. & \left.|\nabla \psi|^{2}+h \mathcal{L}_{\nabla f}+h \mathcal{L}_{\nabla f}^{*}\right) e^{\frac{\psi}{h}} \omega\left|e^{\frac{\psi}{h}} \omega\right\rangle_{\Lambda^{1} L^{2}} \\
& +h \int_{\Gamma_{N D}}\langle\omega \mid \omega\rangle_{\Lambda^{1} T_{\sigma}^{*} \Omega} e^{2 \frac{\psi(\sigma)}{h}}\left(\frac{\partial f}{\partial n}\right)(\sigma) d \sigma
\end{aligned}
$$


holds for any $\omega \in \Lambda^{1} H_{0 ; 0, \mathbf{n}}^{1}\left(\Omega_{U_{1}, \rho}\right)$.

Moreover, when $\omega \in D\left(\Delta_{f, h}^{N, D,(1)}\right)$, the left-hand side equals $\operatorname{Re}\left\langle e^{2 \frac{\psi}{h}} \Delta_{f, h}^{(1)} \omega \mid \omega\right\rangle$.

Proof.

For $\omega$ in $\Lambda^{1} H_{0 ; 0, \mathbf{n}}^{1}\left(\Omega_{U_{1, \rho}}\right)$, we have $\tilde{\omega}:=e^{2 \frac{\psi}{h}} \omega$ in $\Lambda^{1} H_{0 ; 0, \mathbf{n}}^{1}\left(\Omega_{U_{1}, \rho}\right)$ and the same computations as the ones done in [HeNi1] to prove Lemma 4.3 lead to:

$$
\begin{aligned}
\mathcal{D}_{g, f, h}^{N}\left(\omega, e^{\left.2 \frac{\psi}{h} \omega\right)=}\right. & \mathcal{D}_{g, f, h}^{N}(\tilde{\omega}, \tilde{\omega})-\left\langle|\nabla \psi|^{2} \tilde{\omega} \mid \tilde{\omega}\right\rangle \\
& -\left\langle d \psi \wedge \tilde{\omega} \mid d_{f, h} \tilde{\omega}\right\rangle+\left\langle d_{f, h} \tilde{\omega} \mid d \psi \wedge \tilde{\omega}\right\rangle \\
& +\left\langle\mathbf{i}_{\nabla \psi} \tilde{\omega} \mid d_{f, h}^{*} \tilde{\omega}\right\rangle-\left\langle d_{f, h}^{*} \tilde{\omega} \mid \mathbf{i}_{\nabla \psi} \tilde{\omega}\right\rangle .
\end{aligned}
$$

By taking the real part, we obtain:

$$
\operatorname{Re} \mathcal{D}_{g, f, h}^{N}\left(\omega, e^{2 \frac{\psi}{h}} \omega\right)=\mathcal{D}_{g, f, h}^{N}(\tilde{\omega}, \tilde{\omega})-\left\langle|\nabla \psi|^{2} \tilde{\omega} \mid \tilde{\omega}\right\rangle .
$$

We conclude by applying Lemma 2.2.3 .

\subsection{Exponential decay of eigenvectors of $\Delta_{f, h}^{N, D,(1)}$}

As in [HeNi1], the pointwise estimate, $\partial_{x}^{\alpha} u^{h}(x)=\mathcal{O}\left(h^{-N \alpha} e^{-\frac{\Phi(x)}{h}}\right)$, which is stated in Proposition 4.3.1-b), will be proved in several steps. We will first consider $H^{1}$-estimates and deduce afterwards higher order estimates from elliptic regularity.

Even for $H^{1}$-estimates we need two steps: we prove first the exponential decay along the boundary $\Gamma_{N D}$ by applying Lemma 4.3.2 with the function $\psi$ similar to $\varphi$ introduced above ; then the exponential decay in the interior of $\Omega_{U_{1}, \rho}$ is obtained with $\psi$ similar to $\Phi$ once the boundary term is well controlled.

Proof of a) and b) in Proposition 4.3.1.

Statement a)

Actually it is a simple comparison with the full half-space problem via MinMax principle as we did for Theorem 3.1.5. Any $\omega \in \Lambda^{1} H_{0 ; 0, \mathbf{n}}^{1}\left(\Omega_{U_{1}, \rho}\right)$ can indeed be viewed as an element of $\Lambda^{1} H_{0, \mathbf{n}}^{1}\left(\mathbb{R}_{-}^{n}\right)$ by setting $\omega=0$ on $\mathbb{R}_{-}^{n} \backslash \Omega_{U_{1}, \rho}$.

\section{Statement b)}

Let $u^{h} \in D\left(\Delta_{f, h}^{N, D,(1)}\right)$ satisfy

$$
\Delta_{f, h}^{(1)} u^{h}=E(h) u^{h}, E(h)=\mathcal{O}(h),\left\|u^{h}\right\|=1 .
$$

We will use the notation

$$
\tilde{u}^{h}=e^{\frac{\psi^{h}}{h}} u^{h} .
$$


The integration by parts formula (4.3.3) will be applied with $\psi=\psi^{h}$ where $\psi^{h}$ will be similar to $\varphi$ or similar to $\Phi$.

Let us recall

$$
|\nabla f|^{2}=|\nabla \Phi|^{2}, \quad \frac{\partial f}{\partial n}=\frac{\partial \Phi}{\partial n} \quad \text { and } \quad \Phi\left(x^{\prime}, x_{n}\right)=-x_{n}+\varphi\left(x^{\prime}\right),
$$

where $x^{\prime}=0$ is a local minimum for $\varphi$ with $\varphi(0)=0$. Moreover we have $\nabla x_{n} \cdot \nabla \varphi\left(x^{\prime}\right)=0$ so that:

$$
|\nabla f|^{2}=|\nabla \Phi|^{2}=\left|\nabla x_{n}\right|^{2}+|\nabla \varphi|^{2} .
$$

We will first show the decay along the boundary before we propagate the decay in the normal direction inside $\Omega$ (see [HeSj5] and [HeNi1] for references).

Step 1: Decay along $\Gamma_{N D}$.

We take:

$$
\psi^{h}\left(x^{\prime}, x_{n}\right)= \begin{cases}\varphi\left(x^{\prime}\right)-C h \log \frac{\varphi\left(x^{\prime}\right)}{h}, & \text { if } \varphi\left(x^{\prime}\right)>C h \\ \varphi\left(x^{\prime}\right)-C h \log C, & \text { if } \varphi\left(x^{\prime}\right) \leq C h,\end{cases}
$$

where the constant $C>1$ will be fixed later.

We associate the sets:

$$
\Omega_{-}^{h}=\left\{x=\left(x^{\prime}, x_{n}\right) \in \Omega_{U_{1}, \rho} ; \varphi\left(x^{\prime}\right)<C h\right\},
$$

and

$$
\Omega_{+}^{h}=\left\{x=\left(x^{\prime}, x_{n}\right) \in \Omega_{U_{1}, \rho} ; \varphi\left(x^{\prime}\right)>C h\right\} .
$$

The condition $E(h)=\mathcal{O}(h)$ the formula (4.3.3), (4.4.1) and (4.4.2) imply the existence of $C_{1}>0$ such that:

$$
\begin{gathered}
C_{1} h\left\|\tilde{u}^{h}\right\|_{\Lambda^{1} L^{2}\left(\Omega_{-}^{h}\right)}^{2} \geq\left\|h d \tilde{u}^{h}\right\|_{\Lambda^{2} L^{2}}^{2}+\left\|h d^{*} \tilde{u}^{h}\right\|_{\Lambda^{0} L^{2}}^{2}+\left\langle\left|\nabla x_{n}\right|^{2} \tilde{u}^{h} \mid \tilde{u}^{h}\right\rangle_{\Lambda^{1} L^{2}} \\
-h \int_{\Gamma_{N D}}\left\langle\tilde{u}^{h} \mid \tilde{u}^{h}\right\rangle_{\Lambda^{1} T_{\sigma}^{*} \Omega}\left(\frac{\partial x_{n}}{\partial n}\right)(\sigma) d \sigma+\left\langle\left(|\nabla \varphi|^{2}-\left|\nabla \psi^{h}\right|^{2}\right) \tilde{u}^{h} \mid \tilde{u}^{h}\right\rangle \\
-C_{1} h\left\langle 1_{\Omega_{+}^{h}}(x) \tilde{u}^{h} \mid \tilde{u}^{h}\right\rangle,
\end{gathered}
$$

with $C_{1}$ determined by $f$ and the upper bound of $E(h)$.

Furthermore,

$$
\nabla \psi^{h}=\nabla \varphi-1_{\Omega_{+}^{h}}(x) \frac{C h \nabla \varphi}{\varphi},
$$

so we have:

$$
\left|\nabla \psi^{h}\right|^{2}=|\nabla \varphi|^{2}+1_{\Omega_{+}^{h}}(x)\left(-2 C h \frac{|\nabla \varphi|^{2}}{\varphi}+C^{2} h^{2} \frac{|\nabla \varphi|^{2}}{\varphi^{2}}\right) .
$$


Consequently,

$$
\begin{aligned}
C_{1} h\left\|\tilde{u}^{h}\right\|_{\Lambda^{1} L^{2}\left(\Omega_{-}^{h}\right)}^{2} \geq & \left\|h d \tilde{u}^{h}\right\|_{\Lambda^{2} L^{2}}^{2}+\left\|h d^{*} \tilde{u}^{h}\right\|_{\Lambda^{0} L^{2}}^{2}+\left\langle\left|\nabla x_{n}\right|^{2} \tilde{u}^{h} \mid \tilde{u}^{h}\right\rangle_{\Lambda^{1} L^{2}} \\
& -h \int_{\Gamma_{N D}}\left\langle\tilde{u}^{h} \mid \tilde{u}^{h}\right\rangle_{\Lambda^{1} T_{\sigma}^{*} \Omega}\left(\frac{\partial x_{n}}{\partial n}\right)(\sigma) d \sigma \\
& +\left\langle\left[|\nabla \varphi|^{2}\left(\frac{2 C h}{\varphi}-\frac{C^{2} h^{2}}{\varphi^{2}}\right)-C_{1} h\right] 1_{\Omega_{+}^{h}}(x) \tilde{u}^{h} \mid \tilde{u}^{h}\right\rangle .
\end{aligned}
$$

For $x \in \Omega_{+}^{h}$,

$$
\frac{2 C h}{\varphi}-\frac{C^{2} h^{2}}{\varphi^{2}} \geq \frac{C h}{\varphi}\left(\text { since } 2 a-a^{2} \geq a \forall a \in[0,1]\right)
$$

then, $\varphi$ being a positive Morse function, there exists $C_{2}>0$ which is determined by $\varphi$ such that, for all $x \in \Omega_{+}^{h}$,

$$
C_{2} \geq \frac{\left|\nabla \varphi\left(x^{\prime}\right)\right|^{2}}{\varphi\left(x^{\prime}\right)} \geq C_{2}^{-1}
$$

and we get:

$$
\begin{aligned}
C_{1} h\left\|\tilde{u}^{h}\right\|_{\Lambda^{1} L^{2}\left(\Omega_{-}^{h}\right)}^{2} \geq\left\|h d \tilde{u}^{h}\right\|_{\Lambda^{2} L^{2}}^{2} & +\left\|h d^{*} \tilde{u}^{h}\right\|_{\Lambda^{0} L^{2}}^{2} \\
+\left\langle\left|\nabla x_{n}\right|^{2} \tilde{u}^{h} \mid \tilde{u}^{h}\right\rangle_{\Lambda^{1} L^{2}}-h & \int_{\Gamma_{N D}}\left\langle\tilde{u}^{h} \mid \tilde{u}^{h}\right\rangle_{\Lambda^{1} T_{\sigma}^{*} \Omega}\left(\frac{\partial x_{n}}{\partial n}\right)(\sigma) d \sigma \\
& +\left(C C_{2}^{-1}-C_{1}\right) h\left\langle 1_{\Omega_{+}^{h}}(x) \tilde{u}^{h} \mid \tilde{u}^{h}\right\rangle .
\end{aligned}
$$

Since $\partial_{n} f\left(U_{1}\right)=\partial_{n} x_{n}\left(U_{1}\right) \neq 0$, we can choose $\rho$ small enough such that:

$$
C_{3} \geq\left|\nabla x_{n}\right|^{2} \geq C_{3}^{-1} \text { on } \Omega_{U_{1}, \rho},
$$

where $C_{3}$ is a stricly positive constant.

Hence we get, by adding the term $\left(C C_{2}^{-1}-C_{1}\right) h\left\langle 1_{\Omega_{-}^{h}}(x) \tilde{u}^{h} \mid \tilde{u}^{h}\right\rangle$ to (4.4.4):

$$
\begin{aligned}
C C_{2}^{-1} h\left\|\tilde{u}^{h}\right\|_{\Lambda^{1} L^{2}\left(\Omega_{-}^{h}\right)}^{2} & \geq\left\|h d \tilde{u}^{h}\right\|_{\Lambda^{2} L^{2}}^{2}+\left\|h d^{*} \tilde{u}^{h}\right\|_{\Lambda^{0} L^{2}}^{2} \\
+(1+2 \delta(C) h) & \left\langle\left|\nabla x_{n}\right|^{2} \tilde{u}^{h} \mid \tilde{u}^{h}\right\rangle_{\Lambda^{1} L^{2}} \\
& -h \int_{\Gamma_{N D}}\left\langle\tilde{u}^{h} \mid \tilde{u}^{h}\right\rangle_{\Lambda^{1} T_{\sigma}^{*} \Omega}\left(\frac{\partial x_{n}}{\partial n}\right)(\sigma) d \sigma,
\end{aligned}
$$


where $\delta(C)=\frac{1}{2} C_{3}^{-1}\left(C C_{2}^{-1}-C_{1}\right) \rightarrow+\infty$ when $C \rightarrow+\infty$.

At least, we have on $\Omega_{-}^{h}$ by the definitions:

$$
\left|\tilde{u}^{h}\right| \leq e^{C}\left|u^{h}\right| \text { a.e. }
$$

and the condition $\left\|u^{h}\right\|=1$ leads to:

$$
\begin{aligned}
\tilde{\delta}(C) h \geq\left\|h d \tilde{u}^{h}\right\|_{\Lambda^{2} L^{2}}^{2} & +\left\|h d^{*} \tilde{u}^{h}\right\|_{\Lambda^{0} L^{2}}^{2}+(1+2 \delta(C) h)\left\langle\left|\nabla x_{n}\right|^{2} \tilde{u}^{h} \mid \tilde{u}^{h}\right\rangle_{\Lambda^{1} L^{2}} \\
& -h \int_{\Gamma_{N D}}\left\langle\tilde{u}^{h} \mid \tilde{u}^{h}\right\rangle_{\Lambda^{1} T_{\sigma}^{*} \Omega}\left(\frac{\partial x_{n}}{\partial n}\right)(\sigma) d \sigma
\end{aligned}
$$

where $\tilde{\delta}(C)=e^{2 C} C C_{2}^{-1}$.

We now apply (4.3.3) to $\tilde{u}^{h}$ with $\psi=0, f$ and $h$ replaced respectively by $-x_{n}$ and $\frac{h}{1+\delta(C) h}$, in order to get,

$$
\begin{gathered}
(1+\delta(C) h)^{-1}\left\|h d \tilde{u}^{h}\right\|_{\Lambda^{2} L^{2}}^{2}+(1+\delta(C) h)^{-1}\left\|h d^{*} \tilde{u}^{h}\right\|_{\Lambda^{0} L^{2}}^{2} \\
+(1+\delta(C) h)\left\langle\left|\nabla x_{n}\right|^{2} \tilde{u}^{h} \mid \tilde{u}^{h}\right\rangle- \\
h \int_{\Gamma_{N D}}\left\langle\tilde{u}^{h} \mid \tilde{u}^{h}\right\rangle_{\Lambda^{1} T_{\sigma}^{*} \Omega}\left(\frac{\partial x_{n}}{\partial n}\right)(\sigma) d \sigma \\
+h C_{4}\left\|\tilde{u}^{h}\right\|_{\Lambda^{1} L^{2}}^{2} \geq 0
\end{gathered}
$$

with $C_{4}>0$ independent of $C$.

The difference (4.4.5)-(4.4.6) yields:

$$
\begin{aligned}
\frac{\delta(C) h^{3}}{1+\delta(C) h}\left[\left\|d \tilde{u}^{h}\right\|_{\Lambda^{2} L^{2}}^{2}+\left\|d^{*} \tilde{u}^{h}\right\|_{\Lambda^{0} L^{2}}^{2}\right] & -h C_{4}\left\|\tilde{u}^{h}\right\|_{\Lambda^{1} L^{2}}^{2} \\
& +\delta(C) h\left\langle\left|\nabla x_{n}\right|^{2} \tilde{u}^{h} \mid \tilde{u}^{h}\right\rangle \leq \tilde{\delta}(C) h .
\end{aligned}
$$

We choose $C>1$ large enough such that $\delta(C) C_{3}^{-1}-C_{4}>0$.

This leads, after choosing $h_{0}>0$ small enough, to the existence of a constant $C_{5}>0$ such that, for all $h \in\left(0, h_{0}\right]$,

$$
C_{5} h \geq h^{3}\left\|\tilde{u}^{h}\right\|_{\Lambda^{1} H^{1}}^{2} .
$$

Since $\psi^{h} \geq \varphi+\tilde{C} h \log h$ (for all $\tilde{C}>C$ ), we have proved the existence of $N_{0}>0$ such that:

$$
\left\|e^{\frac{\varphi}{h}} u^{h}\right\|_{\Lambda^{1} H^{1}} \leq C_{6} h^{-N_{0}} .
$$


Remember that $\varphi \geq 0$ vanishes only at $x^{\prime}=0$. Using the trace theorem, this also leads to:

$$
\left\|\left.e^{\frac{\varphi}{h}} u^{h}\right|_{\Gamma_{N D}}\right\|_{\Lambda^{1} H^{1 / 2}\left(\Gamma_{N D}\right)} \leq C_{7} h^{-N_{0}} .
$$

Step 2: Normal decay inside $\Omega$.

We follow a very similar approach by working with the function $\Phi$. We take:

$$
\psi^{h}\left(x^{\prime}, x_{n}\right)= \begin{cases}\Phi-C h \log \frac{\Phi}{h}, & \text { if } \Phi>C h \\ \Phi-C h \log C, & \text { if } \Phi \leq C h,\end{cases}
$$

where the constant $C>1$ will be fixed later.

We associate the sets:

$$
\Omega_{-}^{h}=\left\{x=\left(x^{\prime}, x_{n}\right) \in \Omega_{U_{1}, \rho} ; \Phi<C h\right\}
$$

and

$$
\Omega_{+}^{h}=\left\{x=\left(x^{\prime}, x_{n}\right) \in \Omega_{U_{1}, \rho} ; \Phi>C h\right\} .
$$

The formula (4.3.3) is used like in Step 1, with $\tilde{u}^{h}=e^{\frac{\psi^{h}}{h}} u^{h}$ and $E(h)=$ $\mathcal{O}(h)$. The difference comes from the fact that the boundary term is already estimated with (4.4.8).

We have indeed on the boundary $x_{n}=0$ the inequality: $e^{\frac{\psi^{h}}{h}} \leq e^{\frac{\varphi}{h}}$, due to the relation $\left.\Phi\right|_{x_{n}=0}=\varphi$.

From (4.3.3) used like in Step 1 (see (4.4.3)) we get the existence of $C_{1}>0$ such that:

$$
\begin{gathered}
C_{1} h\left\|\tilde{u}^{h}\right\|_{\Lambda^{1} L^{2}\left(\Omega_{-}^{h}\right)}^{2}+C_{1} h\left\|e^{\frac{\varphi}{h} u}\right\|_{H^{1 / 2}\left(\Gamma_{N D} ; \Lambda^{1} T^{*} \Omega_{U_{1}, \rho}\right)}^{2} \geq\left\|h d \tilde{u}^{h}\right\|_{\Lambda^{2} L^{2}}^{2}+\left\|h d^{*} \tilde{u}^{h}\right\|_{\Lambda^{0} L^{2}}^{2} \\
+\left\langle\left(|\nabla f|^{2}-\left|\nabla \psi^{h}\right|^{2}\right) \tilde{u}^{h} \mid \tilde{u}^{h}\right\rangle-C_{1} h\left\langle 1_{\Omega_{+}^{h}}(x) \tilde{u}^{h} \mid \tilde{u}^{h}\right\rangle .
\end{gathered}
$$

Moreover, from (4.4.8) and the inequality

$$
\left|\tilde{u}^{h}(x)\right| \leq e^{C}\left|u^{h}(x)\right| \quad \text { a.e. in } \Omega_{-}^{h},
$$

we get, for any $C>1$, the existence of $\tilde{\delta}(C)>0$ such that the following estimate is satisfied:

$$
\begin{aligned}
& \tilde{\delta}(C) h^{1-2 N_{0}} \geq C_{1} h\left\|\tilde{u}^{h}\right\|_{\Lambda^{1} L^{2}\left(\Omega_{-}^{h}\right)}^{2}+C_{1} h\left\|e^{\frac{\varphi}{h} u}\right\|_{H^{1 / 2}\left(\Gamma_{N D} ; \Lambda^{1} T^{*} \Omega_{U_{1}, \rho}\right)}^{2} \\
& \geq\left\|h d \tilde{u}^{h}\right\|_{\Lambda^{2} L^{2}}^{2}+\left\|h d^{*} \tilde{u}^{h}\right\|_{\Lambda^{0} L^{2}}^{2}+\left\langle\left(|\nabla f|^{2}-\left|\nabla \psi^{h}\right|^{2}\right) \tilde{u}^{h} \mid \tilde{u}^{h}\right\rangle \\
&-C_{1} h\left\langle 1_{\Omega_{+}^{h}}(x) \tilde{u}^{h} \mid \tilde{u}^{h}\right\rangle .
\end{aligned}
$$


Since $|\nabla f|^{2}=|\nabla \Phi|^{2}$ and $\Phi$ is a positive function without critical points, we can use the same computations as the ones done in Step 1 with $\varphi$ replaced by $\Phi$ to get:

$$
\begin{aligned}
|\nabla f|^{2}-\left|\nabla \psi^{h}\right|^{2} & =1_{\Omega_{+}^{h}}(x)\left(2 C h \frac{|\nabla \Phi|^{2}}{\Phi}-C^{2} h^{2} \frac{|\nabla \Phi|^{2}}{\Phi^{2}}\right) \\
& \geq \frac{C h|\nabla \Phi|^{2}}{\Phi} \geq C_{2}^{-1} C h,
\end{aligned}
$$

with $C_{2}>0$ independent of $C$.

We take $C \geq 2 C_{1} C_{2}$. By adding the estimated term $\left(C_{2}^{-1} C-C_{1}\right) h\left\langle 1_{\Omega_{-}^{h}}(x) \tilde{u}^{h} \mid \tilde{u}^{h}\right\rangle$ to (4.4.9) we get:

$$
\tilde{\delta}_{2}(C) h^{1-2 N_{0}} \geq\left\|h d \tilde{u}^{h}\right\|_{\Lambda^{2} L^{2}}^{2}+\left\|h d^{*} \tilde{u}^{h}\right\|_{\Lambda^{0} L^{2}}^{2}+\left(C_{2}^{-1} C-C_{1}\right) h\left\|\tilde{u}^{h}\right\|_{\Lambda^{1} L^{2}},
$$

which gives, by analogy with Step 1 , the existence of $C_{3}>0$ and $N_{1}>0$ such that:

$$
\left\|e^{\frac{\Phi}{h}} u^{h}\right\|_{\Lambda^{1} H^{1}\left(\Omega_{U_{1}}, \rho\right)} \leq C_{3} h^{-N_{1}} .
$$

Step 3: Elliptic regularity.

We now set $\tilde{u}^{h}=e^{\frac{\Phi}{h}} u^{h}$. For $\rho^{\prime}<\rho$, we take a cut-off $\chi \in \mathcal{C}^{\infty}\left(\Omega_{U_{1}, \rho}\right)$ with compact support in $\Omega_{U_{1}, \rho} \cup \Gamma_{N D}$ and such that $\chi=1$ on a neighborhood of $\Omega_{U_{1}, \rho^{\prime}}$. The form $v^{h}=\chi \tilde{u}^{h}$ satisfies the boundary value problem:

$$
\begin{gathered}
\begin{cases}v^{h}-\Delta v^{h}=r_{0}^{h} & \text { in } \mathbb{R}_{-}^{n}, \\
\mathbf{n} v^{h}=0 \text { and } \mathbf{n} d v^{h}=r_{1}^{h} & \text { on }\left\{x_{n}=0\right\}, \\
\text { with }\left\|r_{0}^{h}\right\|_{\Lambda^{1} L^{2}\left(\mathbb{R}_{-}^{n}\right)}=\mathcal{O}\left(h^{-N_{1}}\right) \text { and }\left\|r_{1}^{h}\right\|_{\Lambda^{2} H^{1 / 2}\left(\mathbb{R}^{n-1}\right)}=\mathcal{O}\left(h^{-N_{1}}\right) .\end{cases}
\end{gathered}
$$

This implies, by [Sch], the existence of $N_{2}>0$ such that:

$$
\left\|v^{h}\right\|_{\Lambda^{1} H^{2}}=\mathcal{O}\left(h^{-N_{2}}\right) .
$$

We conclude by induction for any finite decreasing sequence $\left(\rho_{k}\right)_{0 \leq k \leq K}$ with $\rho_{K}>\rho^{\prime}$ and associated cut-offs $\chi_{k}$, with $\chi_{k}=1$ in a neighborhood of $\Omega_{U_{1}, \rho_{k}}$ and $\operatorname{supp} \chi_{k} \subset\left\{\chi_{k-1}=1\right\}$, using the Sobolev injections.

\subsection{Small eigenvalues are exponentially small}

We now check that the eigenvalue $E_{1}(h)$ of $\Delta_{f, h}^{N, D,(1)}$ lying in $\left[0, h^{3 / 2}\right)$ is actually of order $\mathcal{O}\left(e^{-\varepsilon_{\rho} / h}\right)$ for some $\varepsilon_{\rho}>0$. We prove this by comparison 
with the half-space problem as it is done in [Lep1] at the end of the proof of Proposition 4.2.1.

Proof of Proposition 4.3.1-c).

Again we introduce in a neighborhood of $U_{1}$, the coordinate system $\bar{x}=$ $\left(\bar{x}^{\prime}, \bar{x}_{n}\right)$ leading to (3.3.1). The function $f$ and the metric $g_{0}$ are extended according to (3.3.2) and (3.3.3) so that Proposition 3.2.11 can be applied. Consequently, the half-space Witten Laplacian, $\Delta_{\tilde{f}, h}^{N,(1)}$, has a one dimension kernel and its second eigenvalue is larger than $C h^{6 / 5}$.

Let $u^{h}$ be a normalized eigenvector of $\Delta_{f, h}^{N, D,(1)}$ associated with the first eigenvalue $E_{1}(h)$, which belongs to the interval $\left(0, h^{3 / 2}\right]$. Let $\chi \in \mathcal{C}^{\infty}\left(\overline{\Omega_{U_{1}, \rho}}\right)$ be a cut-off function with compact support in $\Omega_{U_{1}, \rho} \cup \Gamma_{N D}$ and such that $\chi=1$ in a neighborhood of 0 with $\left.\frac{\partial \chi}{\partial n}\right|_{\partial \Omega} \equiv 0$.

The form $v^{h}=\chi u^{h} \in \Lambda^{1} H^{2}\left(\mathbb{R}_{-}^{n}\right)$ belongs to the domain of $\Delta_{\tilde{f}, h}^{N,(1)}$, i.e. $\mathbf{n} v^{h}=\mathbf{n} d_{\tilde{f}, h} v^{h}=0$. Moreover, $v^{h}$ satisfies

$$
\left(\Delta_{\tilde{f}, h}^{(1)}-E_{1}(h)\right) v^{h}=-h^{2}[\Delta, \chi] u^{h} \text { in } \mathbb{R}_{-}^{n}
$$

and the 1-form $r^{h}=-h^{2}[\Delta, \chi] u^{h}$ vanishes in a neighborhood $\mathcal{V}_{1}$ of $\bar{x}=0$. Due to the exponential decay of $u^{h}$ stated in Proposition 4.3.1-b), there exist $C$ and $N_{0}$, such that $r^{h}$ also satisfies

$$
\left|r^{h}(\bar{x})\right| \leq C h^{-N_{0}}\left[\sum_{1 \leq|\beta| \leq 2}\left|\partial_{\bar{x}}^{\beta} \chi(\bar{x})\right|\right] e^{-\frac{\Phi(\bar{x})}{h}} \leq e^{-\frac{c_{\chi}}{h}} .
$$

With $\left\|v^{h}\right\|_{\Lambda^{1} L^{2}}=1+\mathcal{O}\left(e^{-c / h}\right),\left\|r^{h}\right\|_{\Lambda^{1} L^{2}}=\mathcal{O}\left(e^{-c / h}\right)$ and the a priori estimate $E_{1}(h)=\mathcal{O}\left(h^{3 / 2}\right)$, the spectral theorem implies $\left|E_{1}(h)-0\right|=\mathcal{O}\left(e^{-c / h}\right)$ like in the proof of Proposition 4.2.1 given in [Lep1].

\subsection{Accurate comparison with the WKB solution}

We now compare the eigenvector associated with an exponentially small eigenvalue with its WKB approximation. We adapt the method presented in [Hel2, HeSj2] and in [HeNi1] by following the same strategy as in Subsection 4.4. The $H^{1}$-estimates are done in two steps with $\psi^{h}$ similar to $\varphi$ and then with $\psi^{h}$ similar to $\Phi$. Finally the elliptic regularity is used for the $\mathcal{C}^{\infty}$-estimates.

Proof of Proposition 4.3.1-d).

Let $u_{1}^{h} \in D\left(\Delta_{f, h}^{N, D,(1)}\right)$ be an eigenvector associated with the first eigenvalue 
$E_{1}(h)$ of $\Delta_{f, h}^{N, D,(1)}$

$$
\Delta_{f, h}^{N, D,(1)} u_{1}^{h}=E_{1}(h) u_{1}^{h},\left\|u_{1}^{h}\right\|=1
$$

According to Proposition 4.3.1-c), we know that $E_{1}(h)=\mathcal{O}\left(e^{-\frac{\varepsilon \rho}{h}}\right)$, with $\varepsilon_{\rho}>0$, while the second eigenvalue of $\Delta_{f, h}^{N, D,(1)}$ is larger than $h^{3 / 2}$.

By taking $\rho>0$ small enough, the WKB approximation $u_{1}^{w k b}$ presented in Subsection 4.2 satisfies

$$
\left\{\begin{array}{l}
\Delta_{f, h}^{(1)} u_{1}^{w k b}=\mathcal{O}\left(h^{\infty}\right) e^{-\frac{\Phi(x)}{h}} \text { in } \Omega_{U_{1}, \rho} \\
\left.\mathbf{n} u_{1}^{w k b}\right|_{\Gamma_{N D}}=0 \\
\left.\mathbf{n} d_{f, h} u_{1}^{w k b}\right|_{\Gamma_{N D}}=0
\end{array}\right.
$$

and there exists $c>0$, such that for any $\rho^{\prime}>0$, we have

$$
\left\|u_{1}^{w k b}\right\|_{\Lambda^{1} L^{2}\left(\Omega_{U_{1}, \rho^{\prime}}\right)} \sim c h^{\frac{n+1}{4}}
$$

(see indeed further the proof of Proposition 5.5.7).

The cut-off function $\chi \in \mathcal{C}^{\infty}\left(\overline{\Omega_{U_{1}, \rho}}\right)$ is supported in $\Omega_{U_{1}, \rho / 2} \cup \Gamma_{N D}$ and satisfies $\chi=1$ on $\Omega_{U_{1}, \rho^{\prime}}$ with $0<\rho^{\prime}<\rho / 2,\left.\frac{\partial \chi}{\partial n}\right|_{\partial \Omega} \equiv 0$. Later, we will take $\rho^{\prime}>0$ small enough, so that $\chi$ can be taken in the form

$$
\chi\left(x^{\prime}, x_{n}\right)=\chi_{1}\left(x^{\prime}\right) \chi_{n}\left(x_{n}\right) .
$$

Like in Lemma 2.3 .5 (replace $1_{[b,+\infty)}(A)$ by $A^{1 / 2} 1_{[b,+\infty)}(A)$ and $\frac{a}{b}$ by $a=$ $O\left(h^{\infty}\right)$ here), the real constant factor $c(h)$ in the truncated WKB approximation $v_{1}^{w k b}=c(h) \chi u_{1}^{w k b}$ can be chosen so that

$$
\left\|v_{1}^{w k b}-u_{1}^{h}\right\|_{\Lambda^{1} H^{1}}=\mathcal{O}\left(h^{\infty}\right)
$$

and, due to the exponential decay of $u_{1}^{h}$ and $u_{1}^{w k b}$,

$$
\left\|\chi\left(u_{1}^{h}-c(h) u_{1}^{w k b}\right)\right\|_{\Lambda^{1} H^{1}}=\mathcal{O}\left(h^{\infty}\right) .
$$

Set

$$
w^{h}=\chi\left(u_{1}^{h}-c(h) u_{1}^{w k b}\right) .
$$

The 1-form $w^{h}$ satisfies in $\Omega_{U_{1}, \rho}$

$$
\begin{aligned}
\left(\Delta_{f, h}^{(1)}-E_{1}(h)\right) w^{h}= & \chi(x)\left(\Delta_{f, h}^{(1)}-E_{1}(h)\right)\left(u_{1}^{h}-c(h) u_{1}^{w k b}\right) \\
& +\left[\Delta_{f, h}^{(1)}, \chi\right]\left(u_{1}^{h}-c(h) u_{1}^{w k b}\right) \\
= & \tilde{r}^{h} e^{-\frac{\Phi(x)}{h}}+r^{h}
\end{aligned}
$$


where $\tilde{r}^{h}$ and $r^{h}$ satisfy, according to Proposition 4.3.1-b),

$$
\tilde{r}^{h}=\mathcal{O}\left(h^{\infty}\right), \quad \operatorname{supp} r^{h} \subset \operatorname{supp} \nabla \chi \quad \text { and } \quad r^{h}=\mathcal{O}\left(h^{-N_{0}}\right) e^{-\frac{\Phi(x)}{h}} .
$$

The last estimate can be done for any $\mathcal{C}^{k_{0}}$-norm, with $k_{0} \in \mathbb{N}$.

On the boundary $\partial \Omega_{U_{1}, \rho}=\Gamma_{N D} \cup \Gamma_{D}$, we have simply

$$
\begin{array}{ll} 
& \left.\mathbf{n} w^{h}\right|_{\Gamma_{N D}}=0,\left.\quad w^{h}\right|_{\Gamma_{D}}=0, \\
\text { and } \quad & \left.\mathbf{n} d_{f, h} w^{h}\right|_{\Gamma_{N D}}=0 .
\end{array}
$$

With the different of choices for $\psi^{h}$ given below, we will use the notation

$$
\tilde{w}^{h}=e^{\frac{\psi^{h}}{h}} w^{h} .
$$

The 1-forms $w$ and $\tilde{w}$ belong to $\Lambda^{1} H^{2}\left(\Omega_{U_{1}, \rho}\right)$ and their supports do not meet $\Gamma_{D}$. Hence the integration by parts formula $(2.2 .7)$ can be used in addition to $(4.3 .3)$.

\section{Step 1: Comparison along $\Gamma_{N D}$.}

Like in the proof of Proposition 4.3.1-b) presented in Subsection 4.4, we introduce the sets

$$
\begin{aligned}
\Omega_{-}^{h} & =\left\{x=\left(x^{\prime}, x_{n}\right) \in \Omega_{U_{1}, \rho} ;\right. & \left.\varphi\left(x^{\prime}\right)<C h\right\}, \\
\text { and } \quad & \Omega_{+}^{h}=\left\{x=\left(x^{\prime}, x_{n}\right) \in \Omega_{U_{1}, \rho} ;\right. & \left.\varphi\left(x^{\prime}\right)>C h\right\} .
\end{aligned}
$$

For any $N \in \mathbb{N}$, we take:

$$
\begin{aligned}
\varphi_{N}^{h}\left(x^{\prime}\right) & =\min \left\{\varphi^{h}\left(x^{\prime}\right)+N h \log h^{-1}, \psi\left(x^{\prime}\right)\right\}, \\
\text { where } \quad \varphi^{h}\left(x^{\prime}\right) & = \begin{cases}\varphi\left(x^{\prime}\right)-C h \log \frac{\varphi\left(x^{\prime}\right)}{h}, & \text { if } \varphi\left(x^{\prime}\right)>C h \\
\varphi\left(x^{\prime}\right)-C h \log C, & \text { if } \varphi\left(x^{\prime}\right) \leq C h,\end{cases} \\
\text { and } \quad \psi\left(x^{\prime}\right) & =\min \left\{\varphi_{-}^{h}\left(y^{\prime}\right)+(1-\varepsilon)\left|\varphi\left(x^{\prime}\right)-\varphi\left(y^{\prime}\right)\right|, y^{\prime} \in \operatorname{supp} \nabla \chi_{1}\right\} .
\end{aligned}
$$

We recall that the cut-off $\chi$ writes $\chi\left(x^{\prime}, x_{n}\right)=\chi_{1}\left(x^{\prime}\right) \chi_{n}\left(x_{n}\right)$. The constant $C \geq 1$ will be fixed at the end like in the proof of Proposition 4.3.1-b). The constants $\rho^{\prime} \in(0, \rho / 2)$ and $\varepsilon>0$ are chosen so that, for $h \in\left(0, h_{N, \rho^{\prime}, \varepsilon}\right)$,

$$
\varphi_{N}^{h}\left(x^{\prime}\right)=\varphi^{h}\left(x^{\prime}\right)+N h \log h^{-1} \text { in } \Omega_{U_{1}, \rho^{\prime}} .
$$

Consequently, $\varphi$ being the Among distance on the boundary,

$$
\varphi_{N}^{h}\left(x^{\prime}\right)=\varphi^{h}\left(x^{\prime}\right)+N h \log h^{-1}=\varphi\left(x^{\prime}\right)-C h \log C+N h \log h^{-1} \quad \text { on } \Omega_{-}^{h} .
$$


Note furthermore the inequalities:

$$
\begin{aligned}
\varphi_{N}^{h}(x) \leq \varphi(x)+N h \log h^{-1} \quad \text { in } \quad \Omega_{U_{1}, \rho} \\
\varphi_{N}^{h}(x) \leq \varphi(x) \leq \Phi(x), \text { if } \quad x^{\prime} \in \operatorname{supp} \nabla \chi_{1}, \\
\text { and } \quad \varphi_{N}^{h}(x) \leq \varphi(x)+N h \log h^{-1} \leq \Phi(x), \text { if } \quad x_{n} \in \operatorname{supp} \chi_{n}^{\prime} .
\end{aligned}
$$

In particular, we have for $h \in\left(0, h_{N, \rho^{\prime}, \varepsilon}\right)$,

$$
\varphi_{N}^{h}(x) \leq \Phi(x), \text { for } x \in \operatorname{supp} \nabla \chi,
$$

which implies

$$
\left\|e^{\frac{\varphi_{N}^{h}}{h}} r^{h}\right\|_{\Lambda^{1} L^{2}}=\mathcal{O}_{N}\left(h^{-N_{0}}\right) .
$$

We apply the integration by parts formula (4.3.3), where the left-hand side is computed with (2.2.7), and we obtain for the form $\tilde{w}^{h}=e^{\frac{\varphi_{N}^{h}}{h}} w^{h}$, by analogy with the proof of Proposition 4.3.1-b), using (4.6.1) and $E_{1}(h)=\mathcal{O}\left(h^{\infty}\right)=$ $\mathcal{O}(h)$ :

$$
\begin{aligned}
C_{1} h\left\|\tilde{w}^{h}\right\|_{\Lambda^{1} L^{2}\left(\Omega_{-}^{h}\right)}+\left\|\tilde{r}^{h}+e^{\frac{\varphi_{N}^{h}(x)}{h}} r^{h}\right\|_{\Lambda^{1} L^{2}}\left\|\tilde{w}^{h}\right\|_{\Lambda^{1} L^{2}} \geq\left\|h d \tilde{w}^{h}\right\|_{\Lambda^{2} L^{2}}^{2}+\left\|h d^{*} \tilde{w}^{h}\right\|_{\Lambda^{0} L^{2}}^{2} \\
+\left\langle\left|\nabla x_{n}\right|^{2} \tilde{w}^{h} \mid \tilde{w}^{h}\right\rangle_{\Lambda^{1} L^{2}}+h \int_{\Gamma_{N D}}\left\langle\tilde{w}^{h} \mid \tilde{w}^{h}\right\rangle_{\Lambda^{1} T_{\sigma}^{*} \Omega}\left(\frac{\partial x_{n}}{\partial n}\right)(\sigma) d \sigma \\
+\left\langle\left(|\nabla \varphi|^{2}-\left|\nabla \varphi_{N}^{h}\right|^{2}\right) \tilde{w}^{h} \mid \tilde{w}^{h}\right\rangle-C_{1} h\left\langle 1_{\Omega_{+}^{h}}(x) \tilde{w}^{h} \mid \tilde{w}^{h}\right\rangle,
\end{aligned}
$$

where the constant $C_{1}>0$ is determined by $f$ and $\tilde{r}^{h}=\mathcal{O}\left(h^{\infty}\right)$.

In $\Omega_{-}^{h}$ the weight $e^{\frac{\varphi_{N}^{h}(x)}{h}}$ is bounded by $C_{2}(C) h^{-N}$ and this provides

$$
\left\|\tilde{w}^{h}\right\|_{\Lambda^{1} L^{2}\left(\Omega_{-}^{h}\right)} \leq C_{2}(C) h^{-N}\left\|w^{h}\right\|_{\Lambda^{1} L^{2}\left(\Omega_{-}^{h}\right)} \leq C_{3}(C, N),
$$

due to $\left\|w^{h}\right\|_{\Lambda^{1} H^{1}}=\mathcal{O}\left(h^{\infty}\right)$.

We obtain:

$$
\begin{aligned}
\tilde{\delta}(C, & N)\left(h^{-N_{0}}\left\|\tilde{w}^{h}\right\|_{\Lambda^{1} H^{1}}+1\right) \geq\left\|h d \tilde{w}^{h}\right\|_{\Lambda^{2} L^{2}}^{2}+\left\|h d^{*} \tilde{w}^{h}\right\|_{\Lambda^{0} L^{2}}^{2} \\
& +\left\langle\left|\nabla x_{n}\right|^{2} \tilde{w}^{h} \mid \tilde{w}^{h}\right\rangle_{\Lambda^{1} L^{2}}+h \int_{\Gamma_{N D}}\left\langle\tilde{w}^{h} \mid \tilde{w}^{h}\right\rangle_{\Lambda^{1} T_{\sigma}^{*} \Omega}\left(\frac{\partial x_{n}}{\partial n}\right)(\sigma) d \sigma \\
& +\left\langle\left(|\nabla \varphi|^{2}-\left|\nabla \varphi_{N}^{h}\right|^{2}\right) \tilde{w}^{h} \mid \tilde{w}^{h}\right\rangle-C_{1} h\left\langle 1_{\Omega_{+}^{h}}(x) \tilde{w}^{h} \mid \tilde{w}^{h}\right\rangle .
\end{aligned}
$$

In $\Omega_{-}^{h},|\nabla \varphi|^{2}=\left|\nabla \psi_{N}^{h}\right|^{2}$, using (4.6.3).

In $\Omega_{+}^{h}$, the point $x$ fulfills almost surely one of the two possibilities: 
- Either $\nabla \varphi_{N}^{h}=\nabla \psi$, and we get

$$
|\nabla \varphi|^{2}-\left|\nabla \psi_{N}^{h}\right|^{2} \geq\left(2 \varepsilon-\varepsilon^{2}\right)|\nabla \varphi|^{2} \geq \delta_{\rho, \varepsilon},
$$

where the last lower bound is due to the fact that $\varphi_{N}(x)=\psi(x)$ cannot occur in a neighborhood of $x^{\prime}=0$ for $\varepsilon>0$ small enough and $h \in\left(0, h_{N, \rho^{\prime}, \varepsilon}\right)$;

- or $\nabla \varphi_{N}^{h}=\nabla \varphi\left(1-\frac{C h}{\varphi}\right)$.

So we get, similarly to the proof of Proposition 4.3.1-b), for $C$ big enough and $h \in\left(0, h_{N, \rho^{\prime}, \epsilon}\right]$, with $h_{N, \rho^{\prime}, \epsilon}>0$ small enough:

$$
\begin{gathered}
\tilde{\delta}_{2}(C, N)\left(h^{-N_{0}}\left\|\tilde{w}^{h}\right\|_{\Lambda^{1} H^{1}}+1\right) \geq \\
\left\|h d \tilde{w}^{h}\right\|_{\Lambda^{2} L^{2}}^{2}+\left\|h d^{*} \tilde{w}^{h}\right\|_{\Lambda^{0} L^{2}}^{2}+(1+2 \delta(C) h)\left\langle\left|\nabla x_{n}\right|^{2} \tilde{w}^{h} \mid \tilde{w}^{h}\right\rangle_{\Lambda^{1} L^{2}} \\
+h \int_{\Gamma_{N D}}\left\langle\tilde{w}^{h} \mid \tilde{w}^{h}\right\rangle_{\Lambda^{1} T_{\sigma}^{*} \Omega}\left(\frac{\partial x_{n}}{\partial n}\right)(\sigma) d \sigma .
\end{gathered}
$$

After treating the r.h.s. like in the proof of Proposition 4.3.1-b)-Step 1, we obtain, for a constant $N_{0}>0$,

$$
\left\|\tilde{w}^{h}\right\|_{\Lambda^{1} H^{1}\left(\Omega_{U_{1}, \rho}\right)} \leq C_{4} h^{-N_{0}} .
$$

Our choice of $\left(\varepsilon, \rho^{\prime}\right)$ imply

$$
\forall x \in \Omega_{U_{1}, \rho^{\prime}}, \quad \varphi_{N}^{h} \geq \varphi(x)+N h \log h^{-1}+\tilde{C} h \log h .
$$

We have proved the existence of $N_{1}$ and $\rho_{0}^{\prime}$, such that, for any $N \in \mathbb{N}$ and $\rho^{\prime} \in\left(0, \rho_{0}^{\prime}\right]$, there exists $h_{N, \rho^{\prime}}>0$ and $C_{N, \rho^{\prime}}>0$, such that:

$$
\left\|e^{\frac{\varphi}{h}}\left(u_{1}^{h}-c(h) u_{1}^{w k b}\right)\right\|_{\Lambda^{1} H^{1}\left(\Omega_{U_{1}, \rho^{\prime}}\right)} \leq C_{N, \rho^{\prime}} h^{N-N_{1}}
$$

holds for any $h \in\left(0, h_{N, \rho^{\prime}}\right)$.

This last estimate and $\left.\Phi\right|_{\Gamma_{N D}}=\varphi$ imply

$$
\left\|e^{\frac{\Phi}{h}}\left(u_{1}^{h}-c(h) u_{1}^{w k b}\right)\right\|_{\Lambda^{1} H^{1 / 2}\left(\Omega_{U_{1}, \rho^{\prime}} \cap \Gamma_{N D}\right)}=\mathcal{O}\left(h^{\infty}\right) .
$$

Step 2: Comparison in the normal direction.

After replacing $\rho^{\prime}$ by $\rho$, Step 1 provides the estimate

$$
\left\|e^{\frac{\varphi}{h}}\left(u_{1}^{h}-c(h) u_{1}^{w k b}\right)\right\|_{\Lambda^{1} H^{1}}=\mathcal{O}\left(h^{\infty}\right) .
$$


We work in $\Omega_{U_{1}, \rho}$ with the above estimate and $\rho^{\prime} \in(0, \rho / 2)$ will be taken again small enough.

In order to get the interior estimate with the weight $e^{\frac{\Phi}{h}}$, we modify the previous analysis like in the proof of Proposition 4.3.1-b). The sets $\Omega_{ \pm}^{h}$ are now given by

$$
\begin{aligned}
\Omega_{-}^{h} & =\left\{x=\left(x^{\prime}, x_{n}\right) \in \Omega_{U_{1}, \rho} ; \Phi<C h\right\}, \\
\text { and } \quad \Omega_{+}^{h} & =\left\{x=\left(x^{\prime}, x_{n}\right) \in \Omega_{U_{1}, \rho} ; \Phi>C h\right\} .
\end{aligned}
$$

The function $\varphi_{N}^{h}, N \in \mathbb{N}$, is given by

$$
\begin{aligned}
\varphi_{N}^{h}(x) & =\min \left\{\varphi^{h}(x)+N h \log h^{-1}, \psi(x)\right\}, \\
\text { with } \quad \varphi^{h}(x) & = \begin{cases}\Phi(x)-C h \log \frac{\Phi(x)}{h}, & \text { if } \Phi>C h, \\
\Phi(x)-C h \log C, & \text { if } \Phi \leq C h,\end{cases} \\
\text { and } \quad \psi(x)=\min \left\{\varphi^{h}(y)+(1-\varepsilon) d_{A g}(x, y), y \in \operatorname{supp} \nabla \chi\right\} . &
\end{aligned}
$$

We recall that the Agmon distance $d_{A g}(x, y)$ is the distance between $x$ and $y$ for the metric $|\nabla f|^{2} d x^{2}$ and $\Phi(x)=d_{A g}\left(x, U_{1}\right)$.

Again, the constant $C \geq 1$ will be fixed in the end like in the proof of Proposition 4.3.1-b), while the constants $\rho^{\prime} \in(0, \rho / 2)$ and $\varepsilon>0$ are chosen so that:

$$
\varphi_{N}^{h}(x)=\varphi^{h}(x)+N h \log h^{-1} \text { in } \Omega_{U_{1}, \rho^{\prime}} .
$$

Again, this implies:

$$
\varphi_{N}^{h}(x)=\varphi^{h}(x)+N h \log h^{-1} \quad \text { on } \Omega_{-}^{h}
$$

Now we have the inequalities

$$
\begin{array}{ll}
\varphi_{N}^{h}(x) \leq \Phi(x)+N h \log h^{-1} \quad \text { in } \Omega_{U_{1}, \rho} \\
\text { and } \quad & \varphi_{N}^{h}(x) \leq \Phi(x) \quad \text { in } \operatorname{supp} \nabla \chi .
\end{array}
$$

Hence the estimate

$$
\left\|e^{\frac{\varphi_{N}^{h}}{h}} r^{h}\right\|_{\Lambda^{1} L^{2}}=\mathcal{O}\left(h^{-N_{0}}\right)
$$

is still valid.

Inequality (4.6.4) implies that the $L^{2}$-norm of the trace of $\tilde{w}^{h}$ on $\Gamma_{N D}$ is $\mathcal{O}\left(h^{\infty}\right)$ and we have the next estimate:

$$
\left\|\tilde{w}^{h}\right\|_{\Lambda^{1} L^{2}\left(\Omega_{-}^{h}\right)} \leq C_{2}(C) h^{-N}\left\|w^{h}\right\|_{\Lambda^{1} L^{2}\left(\Omega_{-}^{h}\right)} \leq C_{3}(C, N) .
$$

With these estimates, the integration by parts formula (4.3.3) and (2.2.7) lead to:

$$
\begin{aligned}
\tilde{\delta}(C, N)\left(h^{-N_{0}}\left\|\tilde{w}^{h}\right\|_{\Lambda^{1} L^{2}}+1\right) & \geq\left\|h d \tilde{w}^{h}\right\|_{\Lambda^{2} L^{2}}^{2}+\left\|h d^{*} \tilde{w}^{h}\right\|_{\Lambda^{0} L^{2}}^{2} \\
& +\left\langle\left(|\nabla \varphi|^{2}-\left|\nabla \varphi_{N}^{h}\right|^{2}-C_{1} h\right) 1_{\Omega_{+}^{h}}(x) \tilde{w}^{h} \mid \tilde{w}^{h}\right\rangle .
\end{aligned}
$$


Finally, for almost all $x \in \Omega_{+}^{h}$ we have:

either: $\nabla \varphi_{N}^{h}(x)=\nabla \psi(x)$

and

$$
|\nabla f|^{2}-\left|\nabla \varphi_{N}^{h}\right|^{2}=\left(2 \varepsilon-\varepsilon^{2}\right)|\nabla f(x)|^{2} \geq \delta_{\rho, \varepsilon}>0 ;
$$

or: $\nabla \varphi_{N}^{h}(x)=\nabla \psi^{h}(x)$

and we get like in the proof of Proposition 4.3.1-b)

$$
|\nabla f|^{2}-\left|\nabla \varphi^{h}\right|^{2} \geq C_{4} C h
$$

By taking $C$ big enough, we get that $\left\|e^{\frac{\varphi_{N}^{h}}{h}} w^{h}\right\|=\mathcal{O}\left(h^{-N_{0}}\right)$ for some $N_{0}>0$. Like in Step 1, this leads to

$$
\left\|e^{\frac{\Phi}{h}}\left(u_{1}^{h}-c(h) u_{1}^{w k b}\right)\right\|_{\Lambda^{1} H^{1}\left(\Omega_{U_{1}, \rho^{\prime}}\right)}=\mathcal{O}\left(h^{\infty}\right),
$$

for $\rho^{\prime} \in(0, \rho / 2)$ small enough.

\section{Step 3:}

The estimates in higher order Sobolev spaces are done like in the proof of Proposition 4.3.1-b) by a bootstrap argument after writing a boundary value problem for $\chi\left(u_{1}^{h}-c(h) u_{1}^{w k b}\right)$ in $\mathbb{R}_{-}^{n}$.

\section{Labelling of local minima and construction of the quasimodes}

\subsection{Preliminaries}

Here we adapt to our case with Neumann boundary condition the method of selecting the proper critical points with index 1 which was used in [HKN] and in [HeNi1]. We recall that the intuition for getting the good labelling of local minima, which is useful even to state properly the assumptions and results, comes from the probabilistic approach. The local minima have to be labelled according to the decreasing order of exit times. We refer to [BGK], [BEGK] and [FrWe] for details.

Note that a similar strategy has independently been considered in [CoPaYc] for the spectral analysis on Markov processes on graphs.

The existence of such a labelling is an assumption which is generically satisfied. After this, it is possible to construct accurately quasimodes leading, with the help of the Witten complex structure, to accurate asymptotic expansions of the low lying eigenvalues. 


\subsection{Generalized critical points and local structure of the level sets of a Morse function}

We recall that we work here on a compact connected oriented Riemannian manifold $\bar{\Omega}=\Omega \cup \partial \Omega$ with boundary and that the function $f$ satisfies Assumption 3.1.1. According to our preliminary results on the Witten Laplacian $\Delta_{f, h}^{N}$ in Theorem 3.1.5, we introduce the following definition of generalized critical points with index $p$.

\section{Definition 5.2.1.}

A point $U \in \bar{\Omega}$ will be called a generalized critical point of $f$ with index $p$ if:

- either $U \in \Omega$ and $U$ is a critical point of $f$ with index $p$,

- or $U \in \partial \Omega$ and $U$ is a critical point with index $p$ of $\left.f\right|_{\partial \Omega}$ such that $\frac{\partial f}{\partial n}(U)<0$ ( $\vec{n}$ being the outgoing normal vector $)$.

Remark 5.2.2. In particular, for $p=0$, we get that the generalized minima are simply the local minima.

The set of generalized critical points with index $p$ is denoted by $\mathcal{U}^{(p)}$. We recall that we want to analyze the Witten Laplacian on 0-forms so we restrict our attention to the cases $p=0$ and $p=1$. From now on, we will use the notation:

$$
m_{p}=\# \mathcal{U}^{(p)} \text { for } p=0,1
$$

instead of $m_{p}^{\bar{\Omega}}$.

Finally it is convenient to call $\mathcal{U}$ the union of all critical points of $f$ and $\left.f\right|_{\partial \Omega}$.

Before labelling the local minima, let us recall a few remarks coming from the local analysis of a Morse function which satisfies Assumption 3.1.1 (we refer to [Mil1], [HKN], and [HeNi1]).

\section{Local structure of the level sets of a Morse function.}

In order to analyze the local situation near a point $x_{0}$ of $\bar{\Omega}$, let us introduce:

$$
A_{f}^{<}\left(x_{0}\right):=\left\{x \in \bar{\Omega} ; f(x)<f\left(x_{0}\right)\right\} \cap B_{x_{0}},
$$

where $B_{x_{0}}$ is a ball centered at $x_{0}$. Similarly, we can introduce

$$
A_{f}^{\leq}\left(x_{0}\right):=\left\{x \in \bar{\Omega} ; f(x) \leq f\left(x_{0}\right)\right\} \cap B_{x_{0}} .
$$

\section{Interior points:}

First we observe that, near a non critical point $x_{0} \in \Omega$ of $f$, one can find $B_{x_{0}}$ and a set of local coordinates such that

$$
A_{f}^{<}\left(x_{0}\right)=\left\{y_{1}<0\right\} \cap B_{x_{0}} .
$$


Secondly, if $x_{0}$ is a critical point with index $p$, then there exists a ball $B_{x_{0}}$ around $x_{0}$ and a set of local coordinates centered at $x_{0}$ such that

$$
A_{f}^{<}\left(x_{0}\right)=\left\{-\sum_{\ell=1}^{p} y_{\ell}^{2}+\sum_{\ell=p+1}^{n} y_{\ell}^{2}<0\right\} \cap B_{x_{0}},
$$

and

$$
A_{f}^{\leq}\left(x_{0}\right)=\left\{-\sum_{\ell=1}^{p} y_{\ell}^{2}+\sum_{\ell=p+1}^{n} y_{\ell}^{2} \leq 0\right\} \cap B_{x_{0}} .
$$

We now observe that

1. When $p=0$ (local minimum), $A_{f}^{<}\left(x_{0}\right)$ is empty and $A_{f}^{\leq}\left(x_{0}\right)$ is reduced to $\left\{x_{0}\right\}$.

2. When $p=1, A_{f}^{<}\left(x_{0}\right)$ has two connected components and $x_{0}$ belongs to the closure of each of the two components. This property will be crucial in the discussion.

3. When $p \geq 2, A_{f}^{<}\left(x_{0}\right)$ is (arcwise) connected.

\section{Points on the boundary:}

If $x_{0}$ belongs to $\partial \Omega$, Assumption 3.1.1 leads to two cases:

\section{First case.}

If $x_{0}$ is not a critical point of $\left.f\right|_{\partial \Omega}$, then the hypersurfaces $\left\{x \mid f(x)=f\left(x_{0}\right)\right\}$ and $\partial \Omega$ intersect transversally in a neighborhood of $x_{0}$. Hence there is a ball $B_{x_{0}}$ around $x_{0}$ and a set of local coordinates such that

$$
A_{f}^{<}\left(x_{0}\right)=\left\{y_{1}<0, y_{n} \leq 0\right\} \cap B_{x_{0}},
$$

and

$$
A_{f}^{\leq}\left(x_{0}\right)=\left\{y_{1} \leq 0, y_{n} \leq 0\right\} \cap B_{x_{0}},
$$

with $\Omega \cap B_{x_{0}}=\left\{y_{n}<0\right\} \cap B_{x_{0}}$.

\section{Second case.}

If $x_{0}$ is a critical point of $\left.f\right|_{\partial \Omega}$ with index $p$ and with $\pm \frac{\partial f}{\partial n}\left(x_{0}\right)>0$, there are local coordinates $\left(y_{1}, \ldots, y_{n-1}, y_{n}\right)$, constructed from the second point of Lemma 3.3.1, such that $\left(y_{1}, \ldots, y_{n-1}\right)$ are Morse coordinates for $\left.f\right|_{\partial \Omega}$ and such that

$$
A_{f}^{<}\left(x_{0}\right)=\left\{ \pm y_{n}-\sum_{i=1}^{p} y_{i}^{2}+\sum_{i=p+1}^{n-1} y_{i}^{2}<0, y_{n} \leq 0\right\} \cap B_{x_{0}},
$$

and

$$
A_{f}^{\leq}\left(x_{0}\right)=\left\{ \pm y_{n}-\sum_{i=1}^{p} y_{i}^{2}+\sum_{i=p+1}^{n-1} y_{i}^{2} \leq 0, y_{n} \leq 0\right\} \cap B_{x_{0}} .
$$

These local models allow to see that 
1. If $x_{0}$ is a local minimum of $\left.f\right|_{\partial \Omega}$ such that $\frac{\partial f}{\partial n}\left(x_{0}\right)<0$, then $A_{f}^{<}\left(x_{0}\right)=\emptyset$ and $A_{f}^{\leq}\left(x_{0}\right)=\left\{x_{0}\right\}$.

2. If $x_{0}$ is a local minimum of $\left.f\right|_{\partial \Omega}$ such that $\frac{\partial f}{\partial n}\left(x_{0}\right)>0$, then $A_{f}^{<}\left(x_{0}\right) \cap \partial \Omega=\emptyset$ and $A_{f}^{\leq}\left(x_{0}\right) \cap \partial \Omega=\left\{x_{0}\right\}$. Moreover, $A_{f}^{<}\left(x_{0}\right)$ is connected.

3. If $p=1$ and $\frac{\partial f}{\partial n}\left(x_{0}\right)<0$ (i.e. if $x_{0} \in \mathcal{U}^{(1)} \cap \partial \Omega$ ), $A_{f}^{<}\left(x_{0}\right)$ has two connected components with a non-empty intersection with $\partial \Omega$ and $x_{0}$ belongs to the closure of each of the two components. Again, this property will be crucial in the discussion.

4. In all other cases, $A_{f}^{<}\left(x_{0}\right)$ is connected with a non-empty intersection with $\partial \Omega$.

\subsection{Labelling of local minima and first consequence}

Remember our main Assumption 1.0.1:

The function $f$ has $\# \mathcal{U}$ distinct critical values and the quantities $f\left(U^{(1)}\right)$ $f\left(U^{(0)}\right)$, with $U^{(1)} \in \mathcal{U}^{(1)}$ and $U^{(0)} \in \mathcal{U}^{(0)}$ are distinct.

Definition 5.3.1. For $\lambda \in \mathbb{R}$, we define $H^{0}(\{f<\lambda\})$ as the number of connected components of the level set $L(\lambda)=f^{-1}((-\infty, \lambda))$.

Due to local structure of the level sets of a Morse function and to Assumption 1.0.1, the function $H^{0}(\{f<\lambda\})$ of $\lambda \in \mathbb{R}$ is a step function which satisfies, with $\lambda$ decreasing from $+\infty$ :

- $H^{0}(\{f<\lambda\})$ decreases by 1 around every $\lambda=f\left(U^{(0)}\right)$ with $U^{(0)} \in$ $\mathcal{U}^{(0)}$.

- wherever $H^{0}(\{f<\lambda\})$ increases by 1 , it is around a $\lambda=f\left(U^{(1)}\right)$ with $U^{(1)} \in \mathcal{U}^{(1)}$.

- $H^{0}(\{f<\lambda\})$ is locally constant away from those points.

Remark 5.3.2. $\bar{\Omega}$ is connected and compact so $H^{0}(\{f<\lambda\})$ equals respectively 1 or 0 for $\lambda \geq \lambda_{f}$ or $\lambda \leq-\lambda_{f}$ for some $\lambda_{f}>0$.

Consequently, the previous discussion implies that the number of critical values of $f$ with index 1 where $H^{0}(\{f<\lambda\})$ increases (by 1$)$ is equal to $m_{0}-1$ and so that $m_{1}+1 \geq m_{0}$.

We now label the local minima of $f$ as follow:

1) We set $U_{1}^{(0)}=\min _{x \in \bar{\Omega}} f, z_{1}=\infty, f\left(z_{1}\right)=z_{1}=\infty$ and we consider $H^{0}(\{f<\lambda\})$ for $\lambda$ decreasing from $f\left(z_{1}\right)=+\infty$. 
2) When $U_{k}^{(0)}$ and $z_{k}$ are defined for $k=1, \ldots, K-1$, decrease $\lambda$ from $f\left(z_{K-1}\right)$ until $H^{0}(\{f<\lambda\})$ increases by 1 . Denote by $\lambda_{K}$ this value.

3) By Assumption 1.0.1 and by the previous discussion, there exists a unique point in $\mathcal{U}^{(1)}$, that we denote by $z_{K}$, satisfying $f\left(z_{K}\right)=\lambda_{K}$. Then we denote by $U_{K}^{(0)}$ the global minimum of the new connected component.

4) We iterate 2) and 3) until all the local minima have been considered.

5) At least we permute the $k$ 's to make the sequence $\left(f\left(z_{k}\right)-f\left(U_{k}^{(0)}\right)\right)_{k \in\left\{1, \ldots, m_{0}\right\}}$ strictly decreasing, which is possible by Assumption 1.0.1.

Definition 5.3.3. (The map j)

If the generalized critical points with index 1 are numbered $U_{j}^{(1)}, j=1, \ldots, m_{1}$, we set $U_{1}^{(1)}=z_{1}=\infty$ and we define the application $k \mapsto j(k)$ from $\left\{1, \ldots, m_{0}\right\}$ to $\left\{0,1, \ldots, m_{1}\right\}$ by:

$$
\left\{\begin{array}{c}
j(1)=0 \text { and } U_{j(1)}^{(1)}=z_{1} \\
\forall k \geq 2, U_{j(k)}^{(1)}=z_{k} .
\end{array}\right.
$$

Definition 5.3.4.

For $k \in\left\{1, \ldots, m_{0}\right\}$, we denote by $E_{k}$ the connected component of $U_{k}^{(0)}$ in

$$
f^{-1}\left(\left(-\infty, f\left(U_{j(k)}^{(1)}\right)\right]\right) \backslash\left\{U_{j(k)}^{(1)}\right\} .
$$

\section{Remark 5.3.5.}

By the previous construction, $U_{k}^{(0)}$ is the global minimum of $E_{k}$.

\section{Proposition 5.3.6.}

Under Assumption 1.0.1, the following properties are satisfied:

a) The sequence $\left(f\left(U_{j(k)}^{(1)}\right)-f\left(U_{k}^{(0)}\right)\right)_{k \in\left\{1, \ldots, m_{0}\right\}}$ is strictly decreasing.

b) $E_{1}=\bar{\Omega}$ is compact and for any $k>1$ the set $E_{k}$ is a relatively compact subset of $f^{-1}\left(\left(-\infty, f\left(U_{j(k)}^{(1)}\right)\right]\right)$ satisfying $\overline{E_{k}}=E_{k} \cup\left\{U_{j(k)}^{(1)}\right\}$.

c) For any $(k, j) \in\left\{1, \ldots, m_{0}\right\} \times\left\{0,1, \ldots, m_{1}\right\}$, the relation $U_{j}^{(1)} \in E_{k}$ implies:

$$
\text { either }\left(j=j\left(k^{\prime}\right) \text { for some } k^{\prime}>k\right) \text { or } j \notin j\left(\left\{1, \ldots, m_{0}\right\}\right) .
$$

d) For any $k \neq k^{\prime} \in\left\{1, \ldots, m_{0}\right\}$, the relation $U_{k^{\prime}}^{(0)} \in E_{k}$ implies:

$$
\left(k^{\prime}>k \quad \text { and } \quad f\left(U_{k^{\prime}}^{(0)}\right)>f\left(U_{k}^{(0)}\right)\right) .
$$

e) The application $j:\left\{1, \ldots, m_{0}\right\} \rightarrow\left\{0,1, \ldots, m_{1}\right\}$ is injective. 
Proof.

By Assumption 1.0.1 and by construction, the points a), b) and e) are obvious.

c) Assume now $U_{j\left(k^{\prime}\right)}^{(1)} \in E_{k}$.

Since $U_{j(k)}^{(1)} \notin E_{k}$, one has $k \neq k^{\prime}$. Moreover, by definition of $E_{k}$ and by Assumption 1.0.1, we have the inequality $f\left(U_{j\left(k^{\prime}\right)}^{(1)}\right)<f\left(U_{j(k)}^{(1)}\right)$ which implies that $E_{k^{\prime}}$ is contained in $E_{k}$, by connectedness of $E_{k}$ and $E_{k^{\prime}}$.

Consequently, $U_{k^{\prime}}^{(0)} \in E_{k}$ and by Assumption 1.0.1, $f\left(U_{k^{\prime}}^{(0)}\right)>f\left(U_{k}^{(0)}\right)$ (because $U_{k}^{(0)}$ is the global minimum of $f$ on $\overline{E_{k}}$ ) which yields:

$$
f\left(U_{j\left(k^{\prime}\right)}^{(1)}\right)-f\left(U_{k^{\prime}}^{(0)}\right)<f\left(U_{j(k)}^{(1)}\right)-f\left(U_{k}^{(0)}\right)
$$

and the point a) gives $k^{\prime}>k$.

d) Assume $U_{k^{\prime}}^{(0)} \in E_{k}$ for $k \neq k^{\prime}$.

Again one has $f\left(U_{k^{\prime}}^{(0)}\right)>f\left(U_{k}^{(0)}\right)$ which implies $k^{\prime} \neq 1\left(\right.$ then $\left.U_{j\left(k^{\prime}\right)}^{(1)} \in \bar{\Omega}\right)$ and there are two possible cases:

$$
U_{j\left(k^{\prime}\right)}^{(1)} \in \overline{E_{k}} \quad \text { or } \quad U_{j\left(k^{\prime}\right)}^{(1)} \notin \overline{E_{k}} .
$$

In the second case, let us look at $E_{k^{\prime}} . E_{k^{\prime}}$ is connected and $U_{k^{\prime}}^{(0)}$ is the global minimum of $f$ on $E_{k^{\prime}}$. Moreover, $U_{k^{\prime}}^{(0)} \in E_{k^{\prime}} \cap E_{k}$ and $U_{j\left(k^{\prime}\right)}^{(1)} \in \overline{E_{k^{\prime}}} \backslash \overline{E_{k}}$ imply, by connectedness, that $\partial E_{k} \cap E_{k^{\prime}} \neq \emptyset$.

$\overline{E_{k}}$ is then contained in $E_{k^{\prime}}$ and $U_{k}^{(0)} \in E_{k^{\prime}}$, which cannot occur.

Consequently, $U_{j\left(k^{\prime}\right)}^{(1)} \in \overline{E_{k}}$ and the points b) and c) imply $k^{\prime}>k$.

\subsection{Construction of the quasimodes}

Like in $[\mathrm{HKN}]$ and in $[\mathrm{HeNi1}]$, we associate with every $U_{k}^{(0)}\left(k \in\left\{1, \ldots, m_{0}\right\}\right)$ a quasimode for $\Delta_{f, h}^{N,(0)}$ which is approximately supported in $E_{k}$, while the quasimodes for $\Delta_{f, h}^{N,(1)}$ will be supported in the balls $B\left(U_{j}^{(1)}, 2 \varepsilon_{1}\right) \quad(j \in$ $\left.\left\{1, \ldots, m_{1}\right\}\right)$. A ball $B(U, \rho)$, with $U \in \bar{\Omega}$ and $\rho>0$, is a geodesic ball and the geodesic distance is denoted by $d_{\Omega}$. The parameter $\varepsilon_{1}>0$ is fixed so that:

- $d_{\Omega}\left(U, U^{\prime}\right) \geq 10 \varepsilon_{1}$ for $U, U^{\prime} \in \mathcal{U}, U \neq U^{\prime}$.

- For all $U \in \mathcal{U}$ and all $k \in\left\{1, \ldots, m_{0}\right\}, U \notin \overline{E_{k}}$ implies

$$
d_{\Omega}\left(U, E_{k}\right) \geq 10 \varepsilon_{1} \text {. }
$$

- The construction of the WKB approximation of Subsection 4.6 is possible in the ball $B\left(U_{j}^{(1)}, 2 \varepsilon_{1}\right)$. If $U_{j}^{(1)}$ is a boundary point, this means 
the introduction of the coordinates $\left(x^{\prime}, x_{n}\right)$ used in Section 4.3 and the existence of $\Phi$. Recall that in these coordinates, $\Phi$ and $g_{0}$ have the form:

$$
\Phi=-x_{n}+\varphi\left(x^{\prime}\right) \quad \text { and } \quad g_{0}=g_{n n}(x) d x_{n}^{2}+\sum_{i, j=1}^{n-1} g_{i j}(x) d x_{i} d x_{j}
$$

The parameter $\varepsilon_{1}>0$ will be kept fixed, while we need another parameter $\varepsilon \in\left(0, \varepsilon_{0}\right)$ which will be fixed in the final step of the proof.

Like in [HeNi1], the construction presented in [HKN] has to be adapted when $U_{j(k)}^{(1)} \in \partial \Omega$ or $U_{k}^{(0)} \in \partial \Omega$ (recall that in [HeNi1], the case $U_{k}^{(0)} \in \partial \Omega$ did not occur) and we focus on these changes.

However, note that in [HeNi1] the set $\overline{E_{k}}$ intersected $\partial \Omega$ at most at one point $\left(\overline{E_{k}} \cap \partial \Omega \subset\left\{U_{j(k)}^{(1)}\right\}\right)$. It is not the case here and we cannot use the same construction when $U_{j(k)}^{(1)} \in \partial \Omega$.

For every $k \in\left\{1, \ldots, m_{0}\right\}$ and $\varepsilon>0$, we introduce the set:

$$
\widetilde{\Omega}_{k}(\varepsilon, \delta)=\left\{x \in \bar{\Omega}, d_{\Omega}\left(x, \overline{E_{k}} \backslash B\left(U_{j(k)}^{(1)}, \varepsilon\right)\right)<\delta\right\} \cup B\left(U_{j(k)}^{(1)}, \varepsilon\right),
$$

with $\delta \in\left(0, \delta_{\varepsilon}\right), \delta_{\varepsilon}>0$ small enough.

The cut-off function $\tilde{\chi}_{k, \varepsilon} \in \mathcal{C}_{0}^{\infty}(\bar{\Omega}), 0 \leq \tilde{\chi}_{k, \varepsilon} \leq 1$ is chosen so that:

$$
\operatorname{supp} \tilde{\chi}_{k, \varepsilon} \subset \widetilde{\Omega}_{k}\left(\varepsilon, \delta_{\varepsilon}\right) \quad \text { and }\left.\quad \tilde{\chi}_{k, \varepsilon}\right|_{\widetilde{\Omega}_{k}\left(\varepsilon, \delta_{\varepsilon} / 2\right) \backslash B\left(U_{j(k)}^{(1)}, \varepsilon\right)}=1
$$

Around $U_{j(k)}^{(1)}$, the cut-off function $\tilde{\chi}_{k, \varepsilon}$ is chosen (more accurately below when $\left.U_{j(k)}^{(1)} \in \partial \Omega\right)$ so that $U_{j(k)}^{(1)} \notin \operatorname{supp} \tilde{\chi}_{k, \varepsilon}$ and

$$
\forall x \in B\left(U_{j(k)}^{(1)}, \varepsilon\right),\left(\tilde{\chi}_{k, \varepsilon}(x) \neq 0 \text {, and } f(x)<f\left(U_{j(k)}^{(1)}\right)\right) \Rightarrow x \in E_{k} .
$$

Remark 5.4.1. The cut-off functions $\tilde{\chi}_{k, \varepsilon}$ are used in the construction of quasimodes for $\Delta_{f, h}^{N,(0)}$.

Moreover, in the case $k=1$, we have by construction $\tilde{\chi}_{k, \varepsilon} \equiv 1$ in $\bar{\Omega}$ because $U_{j(1)}^{(1)} \notin \bar{\Omega}$. This case provides directly the eigenvector $\left\|e^{-f(x) / h}\right\|^{-1} e^{-f(x) / h}$ (of $\Delta_{f, h}^{N,(0)}$ ) with the eigenvalue 0 .

Like in $[\mathrm{HKN}]$ and in [HeNi1], we deduce from Proposition 5.3.6 the following properties for $\tilde{\chi}_{k, \varepsilon}$. 


\section{Proposition 5.4.2.}

By taking $\delta=\delta_{\varepsilon}$ with $\varepsilon \in\left(0, \varepsilon_{0}\right], 0<\varepsilon_{0} \leq \varepsilon_{1}$ small enough, the cut-off functions $\tilde{\chi}_{k, \varepsilon}\left(k \in\left\{1, \ldots, m_{0}\right\}\right)$ satisfy the following properties:

a) If $x$ belongs to $\operatorname{supp} \tilde{\chi}_{k, \varepsilon}$ and $f(x)<f\left(U_{j(k)}^{(1)}\right)$, then $x \in E_{k}$.

b) There exist $C>0$ and, for any $\varepsilon \in\left(0, \varepsilon_{0}\right]$, a constant $K_{\varepsilon}>0$, such that, for $x \in \operatorname{supp} \nabla \tilde{\chi}_{k, \varepsilon}$,

$$
\begin{aligned}
& \text { either } x \notin B\left(U_{j(k)}^{(1)}, \varepsilon\right) \quad \text { and } \quad f\left(U_{j(k)}^{(1)}\right)+K_{\varepsilon}^{-1} \leq f(x) \leq f\left(U_{j(k)}^{(1)}\right)+K_{\varepsilon}, \\
& \text { or } \quad x \in B\left(U_{j(k)}^{(1)}, \varepsilon\right) \quad \text { and } \quad\left|f(x)-f\left(U_{j(k)}^{(1)}\right)\right| \leq C \varepsilon .
\end{aligned}
$$

c) For any $U \in \mathcal{U}, U \neq U_{j(k)}^{(1)}$, the distance $d_{\Omega}\left(U, \operatorname{supp} \nabla \tilde{\chi}_{k, \varepsilon}\right)$ is bounded from below by $3 \varepsilon_{1}>0$. If in addition $U \in \operatorname{supp} \tilde{\chi}_{k, \varepsilon}$, then $U \in E_{k}$.

d) If, for some $k^{\prime} \in\left\{1, \ldots, m_{0}\right\}, U_{k^{\prime}}^{(0)}$ belongs to $\operatorname{supp} \tilde{\chi}_{k, \varepsilon}$, then $k^{\prime} \geq k$ and

$$
f\left(U_{k^{\prime}}^{(0)}\right)>f\left(U_{k}^{(0)}\right), \quad f\left(U_{j\left(k^{\prime}\right)}^{(1)}\right) \leq f\left(U_{j(k)}^{1}\right), \quad \text { if } k \neq k^{\prime} .
$$

e) For any $j \in\left\{1, \ldots, m_{1}\right\}$, such that $U_{j}^{(1)} \in \operatorname{supp} \tilde{\chi}_{k, \varepsilon}$,

$$
\begin{aligned}
& \text { either } j \notin j\left(\left\{1, \ldots, m_{0}\right\}\right), \\
& \text { or } \quad j=j\left(k^{\prime}\right), \text { for some } k^{\prime} \geq k \text { and } U_{k^{\prime}}^{(0)} \in \operatorname{supp} \tilde{\chi}_{k, \varepsilon} .
\end{aligned}
$$

The quasimodes for $\Delta_{f, h}^{N,(1)}$ associated with the $U_{j}^{(1)} \in \Omega$ are constructed like in $[\mathrm{HKN}]$ and in [HeNi1] (and rely on the approximation by the Dirichlet problem in small balls $\left.B\left(U_{j}^{(1)}, 2 \varepsilon_{1}\right)\right)$. We will not recall the complete construction here.

In the same spirit as in [HeNi1], the quasimodes associated with the $U_{j}^{(1)} \in$ $\partial \Omega$ will rely on the approximation by the Neumann realization associated with the neighborhood $\Omega_{U_{j}^{(1)}, \rho}(\rho>0$ small enough $)$ which was studied in Subsection 4.6.

Once $\rho>0$ is fixed uniformly for all $U_{j}^{(1)} \in \partial \Omega$, the parameter $\varepsilon_{1}>0$ is reduced so that $B\left(U_{j}^{(1)}, 2 \varepsilon_{1}\right) \subset \Omega_{U_{1}, \rho}$ for all $U_{j}^{(1)} \in \partial \Omega$.

For all $j \in\left\{1, \ldots, m_{1}\right\}, u_{j}$ denotes a normalized eigenvector associated with the first (exponentially small) eigenvalue of this Dirichlet or Neumann realization. The cut-off function $\theta_{j} \in \mathcal{C}_{0}^{\infty}\left(B\left(U_{j}^{(1)}, 2 \varepsilon_{1}\right)\right)$ is taken such that $\theta_{j}=1$ on $B\left(U_{j}^{(1)}, \varepsilon_{1}\right)$ and $\left.\frac{\partial \theta_{j}}{\partial n}\right|_{\partial \Omega} \equiv 0$ for boundary points $U_{j}^{(1)} \in \partial \Omega$.

Note that the function $\tilde{\chi}_{k, \varepsilon}$ depends on $\varepsilon \in\left(0, \varepsilon_{0}\right]$, while $\theta_{j}$ is kept fixed like $\varepsilon_{1}>0$. 
Definition 5.4.3. For cut-off $\chi_{k, \varepsilon}$ satisfying the properties of Proposition 5.4.2 like $\tilde{\chi}_{k, \varepsilon}$, introduce the following quasimodes.

For any $k \in\left\{1, \ldots, m_{0}\right\}$, the $(\varepsilon, h)$-dependent function $\psi_{k}^{(0)}$ is defined by

$$
\psi_{k}^{(0)}(x)=\left\|\chi_{k, \varepsilon}(x) e^{-\left(f(x)-f\left(U_{k}^{(0)}\right)\right) / h}\right\|^{-1} \chi_{k, \varepsilon}(x) e^{-\left(f(x)-f\left(U_{k}^{(0)}\right)\right) / h} .
$$

For any $j \in\left\{1, \ldots, m_{1}\right\}$, the $h$-dependent 1 -form $\psi_{j}^{(1)}$ is defined by

$$
\psi_{j}^{(1)}(x)=\left(\left\|\theta_{j} u_{j}\right\|^{-1}\right) \theta_{j}(x) u_{j}(x) .
$$

We set $\lambda_{1}^{a p p}(\varepsilon, h)=0$, and for any $k \in\left\{2, \ldots, m_{0}\right\}$ :

$$
\lambda_{k}^{a p p}(\varepsilon, h)=\left|\left\langle\psi_{j(k)}^{(1)} \mid d_{f, h}^{(0)} \psi_{k}^{(0)}\right\rangle\right|^{2} .
$$

\section{Remark 5.4.4.}

a) In the case $U_{j(k)}^{(1)} \in \Omega, \chi_{k, \varepsilon}$ is $\tilde{\chi}_{k, \varepsilon}$ with additional properties (see [HKN] for details) and we will still denote it here by $\tilde{\chi}_{k, \varepsilon}$. In the case $U_{j(k)}^{(1)} \in \partial \Omega$, the real choice of $\chi_{k, \varepsilon}$ will be fixed further (see Definition 5.5.8). Moreover, $\chi_{k, \varepsilon}$ also satisfies the properties of Proposition 5.4.2.

b) For the sake of conciseness, we omit the $(\varepsilon, h)$ - and $h$-dependence in the notations $\psi_{k}^{(0)}$ and $\psi_{j}^{(1)}$.

c) We will show in the next section that the $\lambda_{k}^{a p p}(\varepsilon, h)$ 's are approximated values of the small eigenvalues of $\Delta_{f, h}^{N,(0)}$.

By Remark 5.4.1, this definition is coherent for $k=1$ and $\psi_{1}^{(0)}$ is the normalized eigenvector associate with the eigenvalue 0.

d) Due to the condition $\left.\frac{\partial \theta_{j}}{\partial n}\right|_{\partial \Omega} \equiv 0, \psi_{j}^{(1)}$ belongs to $D\left(\Delta_{f, h}^{N,(1)}\right)$ and this, even if $U_{j}^{(1)}$ belongs to $\partial \Omega$.

\subsection{Quasimodal estimates}

We end this section by reviewing the quasimodal estimates which are derived from Propositions 5.3.6 and 5.4.2. The asymptotic expansion of the quantity $\left\langle\psi_{j(k)}^{(1)} \mid d_{f, h}^{(0)} \psi_{k}^{(0)}\right\rangle$ has also be done in $[\mathrm{HKN}]$ when $U_{k}^{(0)}$ and $U_{j(k)}^{(1)} \in \Omega$ are interior points. Like in [HeNi1], we will simply complete this analysis by establishing the asymptotic expansion of $\left\langle\psi_{j(k)}^{(1)} \mid d_{f, h}^{(0)} \psi_{k}^{(0)}\right\rangle$, when $U_{k}^{(0)}$ or $U_{j(k)}^{(1)}$ is in $\partial \Omega$.

Remark 5.5.1. In this subsection, we make computations with different coordinate systems $v=\left(v_{1}, \ldots, v_{n}\right)$ (around $U=U_{k}^{(0)}$ or $\left.U=U_{j(k)}^{(1)}\right)$ all given given by Lemma 3.3.1. 
Looking at the proof of Lemma 3.3.1 given in [Lep1], notice that the coordinates $\left(v_{1}, \ldots, v_{n-1}\right)$ in the boundary can be chosen freely. Moreover, according to [HeSj4] pp. 279-280, they can be chosen such that $d v_{1}(U), \ldots, d v_{n-1}(U), \vec{n}_{U}^{*}$ is orthonormal and positively oriented and

$f(v, 0)=\frac{\lambda_{1}}{2} v_{1}^{2}+\cdots+\frac{\lambda_{n-1}}{2} v_{n-1}^{2}+f(U) \quad$ and $\quad \varphi(v)=\frac{\left|\lambda_{1}\right|}{2} v_{1}^{2}+\cdots+\frac{\left|\lambda_{n-1}\right|}{2} v_{n-1}^{2}$,

with $\lambda_{1}<0$ when $U=U_{j}^{(1)}$. Hence all the coordinates systems around $U \in \partial \Omega$ will coincide on $\partial \Omega$ while they may differ in $\Omega$ according to the case when a normal form is used for $f, \Phi$ or $f+\Phi$ in $\Omega$.

Remind that the parameter $\varepsilon_{1}>0$ is fixed, while $\varepsilon_{0}$ and $\varepsilon \in\left(0, \varepsilon_{0}\right]$ may have to be adapted during the proof. We shall denote by $\alpha$ a generic positive constant which is independent of $\varepsilon \in\left(0, \varepsilon_{0}\right]$.

Introduce the next notation which will be very useful:

Definition 5.5.2. The notation $g(h)=\mathcal{O}_{\varepsilon}\left(e^{-\frac{\alpha}{h}}\right)$ means that, for all $\varepsilon \in$ $\left(0, \varepsilon_{0}\right]$, there exists a constant $C_{\varepsilon}>0$ such that:

$$
\forall h \in\left(0, h_{0}\right], \quad|g(h)| \leq C_{\varepsilon} e^{-\frac{\alpha}{h}} .
$$

From Proposition 5.3.6-d) and the good localization of $\nabla \chi_{k, \varepsilon}$, we deduce the following estimates for $\psi_{k}^{(0)}$.

\section{Proposition 5.5.3.}

The system of $(\varepsilon, h)$-dependent functions $\left(\psi_{k}^{(0)}\right)_{k \in\left\{1, \ldots, m_{0}\right\}}$ of Definition 5.4 .3 is almost orthogonal with

$$
\left(\left\langle\psi_{k}^{(0)} \mid \psi_{k^{\prime}}^{(0)}\right\rangle\right)_{k, k^{\prime} \in\left\{1, \ldots, m_{0}\right\}}=\operatorname{Id}_{\mathbb{C}^{m_{0}}}+\mathcal{O}_{\varepsilon}\left(e^{-\frac{\alpha}{h}}\right),
$$

and there exists $\alpha>0$ and, for any $\varepsilon \in\left(0, \varepsilon_{0}\right], C(\varepsilon)$ and $h_{0}(\varepsilon)$ such that, for any $h \in\left(0, h_{0}(\varepsilon)\right]$,

$$
\left\langle\Delta_{f, h}^{N,(0)} \psi_{k}^{(0)} \mid \psi_{k}^{(0)}\right\rangle=\left\|d_{f, h}^{(0)} \psi_{k}^{(0)}\right\|^{2} \leq C(\varepsilon) e^{-2 \frac{f\left(U_{j(k)}^{(1)}\right)-f\left(U_{k}^{(0)}\right)-\alpha \varepsilon}{h}} .
$$

\section{Corollary 5.5.4.}

There exists $\varepsilon_{0}>0$ and $\alpha>0$ such that, for any choice of $\varepsilon$ in $\left(0, \varepsilon_{0}\right]$ and for all $k \in\left\{1, \ldots, m_{0}\right\}$, the $(\varepsilon, h)$-dependent quasimodes $\psi_{k}^{(0)}$ satisfy the estimate

$$
\left\langle\Delta_{f, h}^{N,(0)} \psi_{k}^{(0)} \mid \psi_{k}^{(0)}\right\rangle=\mathcal{O}_{\varepsilon}\left(e^{-\frac{\alpha}{h}}\right) .
$$

The exponential decay of the first eigenvector $u_{j}$, associated with an exponentially small eigenvalue, of the Dirichlet realization of $\Delta_{f, h}^{(1)}$ around $U_{j}^{(1)}$, provides the next estimates for $\psi_{j}^{(1)}$. We refer the reader to [HKN] or [HeSj4] for $U_{j}^{(1)} \in \Omega$ and to Subsection 4.6 for $U_{j}^{(1)} \in \partial \Omega$. 


\section{Proposition 5.5.5.}

The system of h-dependent 1 -forms, $\left(\psi_{j}^{(1)}\right)_{j \in\left\{1, \ldots, m_{1}\right\}}$ given in Definition 5.4.3 is orthonormal and there exists $\alpha>0$ independent of $\varepsilon$ such that

$$
\left\langle\Delta_{f, h}^{N,(1)} \psi_{j}^{(1)} \mid \psi_{j}^{(1)}\right\rangle=\mathcal{O}\left(e^{-\frac{\alpha}{h}}\right),
$$

for all $j \in\left\{1, \ldots, m_{1}\right\}$.

Let us now compute some asymptotic expansions.

Proposition 5.5.6. For $k$ in $\left\{2, \ldots, m_{0}\right\}$ and $x$ in $\bar{\Omega}$,

$$
\psi_{k}^{(0)}(x)=\gamma_{k}(h)\left(1+a_{k}(h)\right) \chi_{k, \varepsilon}(x) e^{-\frac{f(x)-f\left(U_{k}^{(0)}\right)}{h}},
$$

where $\gamma_{k}(h)$ is defined in Definition 1.0.2 and $a_{k}(h) \sim \sum_{\ell=1}^{\infty} a_{k, \ell} h^{\ell}$.

Proof. In the case $U_{k}^{(0)} \in \Omega$, we refer the reader to [HKN].

If $U_{k}^{(0)} \in \partial \Omega$, we use again, in a neighborhood of $U_{k}^{(0)}$, the coordinate system $\left(\bar{x}^{\prime}, \bar{x}_{n}\right)$ introduced in the second part of the Section $3.3\left(\right.$ with $\left.\bar{x}\left(U_{k}^{(0)}\right)=0\right)$. In this coordinate system, $f$ and $g_{0}$ equal:

$$
\begin{gathered}
f(\bar{x})=-\bar{x}_{n}+\left.f\right|_{\partial \Omega}\left(\bar{x}^{\prime}\right)=-\bar{x}_{n}+f\left(U_{k}^{(0)}\right)+\varphi\left(\bar{x}^{\prime}\right), \\
g_{0}=g_{n n}(\bar{x}) d \bar{x}_{n}^{2}+\sum_{i, j=1}^{n-1} g_{i j}(\bar{x}) d \bar{x}_{i} d \bar{x}_{j},
\end{gathered}
$$

where $\varphi=\left.f\right|_{\partial \Omega}-f\left(U_{k}^{(0)}\right)$ is the Agmon distance to $U_{k}^{(0)}$ on the boundary. We denote by $V_{g_{0}}(d \bar{x})$ the normalized volume form:

$$
V_{g_{0}}(d \bar{x})=\left(\operatorname{det} G_{0}(\bar{x})\right)^{1 / 2} d \bar{x}^{\prime} \wedge d \bar{x}_{n}=: \nu\left(\bar{x}^{\prime}, \bar{x}_{n}\right) d \bar{x}^{\prime} \wedge d \bar{x}_{n} .
$$

From (5.5.2),

$$
d \bar{x}_{n}\left(U_{k}^{(0)}\right)=-\frac{\partial f}{\partial n}\left(U_{k}^{(0)}\right) \vec{n}_{U_{k}^{(0)}}^{(0)} \text { and } \nu(0,0)=\left(-\frac{\partial f}{\partial n}\left(U_{k}^{(0)}\right)\right)^{-1} .
$$

For some constants $\eta>0$ and $\delta_{\eta}>0$,

$$
\begin{aligned}
\left\|\chi_{k, \varepsilon} e^{-\frac{f(x)-f\left(U_{k}^{(0)}\right)}{h}}\right\|^{2} & =\int_{\bar{\Omega}} \chi_{k, \varepsilon}^{2} e^{-2 \frac{f(x)-f\left(U_{k}^{(0)}\right)}{h}} V_{g_{0}}(d x) \\
& =\int_{B(0, \eta)} e^{2 \frac{\bar{x}_{n}}{h}} e^{-2 \frac{\varphi\left(\bar{x}^{\prime}\right)}{h}} \nu\left(\bar{x}^{\prime}, \bar{x}_{n}\right) d \bar{x}^{\prime} \wedge d \bar{x}_{n}+\mathcal{O}\left(e^{-\frac{\delta_{\eta}}{h}}\right) .
\end{aligned}
$$

According to (5.5.1),

$$
\begin{aligned}
& \left\|\chi_{k, \varepsilon} e^{-\frac{f(x)-f\left(U_{k}^{(0)}\right)}{h}}\right\|^{2}=\int_{B(0, \eta)} e^{2 \frac{\bar{x}_{n}}{h}} e^{-\frac{\left|\lambda_{1}\right| \bar{x}_{1}^{2}+\cdots+\left|\lambda_{n-1}\right| \bar{x}_{n-1}^{2}}{h}} \nu\left(\bar{x}^{\prime}, \bar{x}_{n}\right) d \bar{x} \wedge d \bar{x}_{n} \\
& +\mathcal{O}\left(e^{-\frac{\delta_{\eta}}{h}}\right) .
\end{aligned}
$$


By expanding $\nu\left(\bar{x}^{\prime}, \bar{x}_{n}\right)$ to a Taylor Series of arbitrary order $k \in \mathbb{N}^{*}$, we can separate the variables $\bar{x}^{\prime}$ and $\bar{x}_{n}$ in the last integral term.

Hence, using the Laplace Method for each term, we obtain an asymptotic expansion of arbitrary order of $\left\|\chi_{k, \varepsilon} e^{-\frac{f(x)-f\left(U_{k}^{(0)}\right)}{h}}\right\|^{2}$.

Moreover, from (5.5.4), the first term is:

$$
\left(-\frac{\partial f}{\partial n}\left(U_{k}^{(0)}\right)\right)^{-1} \frac{h}{2} \frac{(\pi h)^{\frac{n-1}{2}}}{\left.|\operatorname{det} \operatorname{Hess} f|_{\partial \Omega}\left(U_{k}^{(0)}\right)\right|^{\frac{1}{2}}}=\left(\gamma_{k}(h)\right)^{-2} .
$$

Proposition 5.5.7. In $B\left(U_{j(k)}^{(1)}, \varepsilon_{1}\right)$, choose the coordinate system $x$ which satisfies (5.4.1) and (5.5.1) with $\lambda_{1}<0$. For $k$ in $\left\{2, \ldots, m_{0}\right\}$, the equality

$$
\psi_{j(k)}^{(1)}(x)=\delta_{j(k)}(h) b(x, h) e^{-\frac{\Phi(x)}{h}},
$$

holds up to a phase factor, when $\delta_{j(k)}(h)$ is defined according to Definition 1.0.2, $b(x, h) \sim \sum_{\ell=0}^{\infty} b_{k, \ell}(x) h^{\ell}, b_{k, \ell}(x)=\sum_{i=1}^{n} b_{k, \ell}^{i}(x) d x_{i}$, and $b_{k, 0}^{i}(0)=$ $\delta_{1 i}$.

Proof. In Section 4, we found a WKB approximation $u_{1}^{w k b}$ of an eigenvector $u_{1}^{h}$ such that,

$$
\begin{aligned}
& e^{\frac{\Phi(x)}{h}} u_{1}^{w k b}=\sum_{i=1}^{n} a_{i}^{0}(x) d x_{i}+h a^{1}(x, h), \\
& a_{i}^{0}(0)=\delta_{1 i}, a^{1}(x, h) \sim \sum_{\ell} h^{\ell} a_{\ell}(x),
\end{aligned}
$$

and

$$
\forall x \in B\left(U_{j(k)}^{(1)}, 2 \varepsilon_{1}\right), \quad e^{\frac{\Phi(x)}{h}}\left|\partial_{x}^{\alpha}\left(u_{1}^{h}(x)-u_{1}^{w k b}(x)\right)\right| \leq C_{\alpha, N} h^{N} .
$$

The WKB approximation $u_{1}^{w k b}$ was initially constructed in another coordinate system $\left(\underline{x}_{1}, \ldots, \underline{x}_{n}\right)$. Remark 5.5 .1 recalls that the tangential coordinates $\underline{x}_{1}, \ldots, \underline{x}_{n-1}$ and $x_{1}, \ldots, x_{n-1}$ can coincide in $\partial \Omega$ with different deformations as entering into $\Omega$.

The normalized eigenvector that we take here is

$$
u_{j(k)}=\frac{u_{1}^{h}}{\left\|u_{1}^{h}\right\|} .
$$

Let us first compute accurately:

$$
\left\|u_{1}^{h}\right\|=\left\|\theta_{j(k)} u_{1}^{h}\right\|+\mathcal{O}\left(h^{\infty}\right)=\left\|\theta_{j(k)} u_{1}^{w k b}\right\|+\mathcal{O}\left(h^{\infty}\right) .
$$


Moreover,

$$
\left\|\theta_{j(k)} u_{1}^{w k b}\right\|^{2}=\int \theta_{j(k)}(x)^{2}\langle a(x, h) \mid a(x, h)\rangle e^{-\frac{2 \Phi(x)}{h}} V_{g_{0}}(d x),
$$

where the integral is over $x_{n} \leq 0$. Note furthermore that,

$$
d x_{n}\left(U_{j(k)}^{(1)}\right)=-\frac{\partial \Phi}{\partial n}\left(U_{j(k)}^{(1)}\right) \vec{n}_{U_{j(k)}^{(1)}}^{(1)}=-\frac{\partial f}{\partial n}\left(U_{j(k)}^{(1)}\right) \vec{n}_{U_{j(k)}^{(1)}}^{(.)} .
$$

Proceeding like in the proof of Proposition 5.5.6, we obtain, using the Laplace method, a full asymptotic expansion of $\left\|\theta_{j(k)} u_{1}^{w k b}\right\|^{2}$. The first term is given by the first term of

$$
\int \theta_{j(k)}(x)^{2}\left\langle a^{0}(x) \mid a^{0}(x)\right\rangle e^{\frac{2 x_{n}}{h}} e^{-2 \frac{\varphi\left(x^{\prime}\right)}{h}} V_{g_{0}}(d x),
$$

and from $\left\langle a^{0}(x) \mid a^{0}(x)\right\rangle(0)=1$, we conclude like in the proof of Proposition 5.5.6.

Before stating the next result, let us specify the choice of $\chi_{k, \varepsilon}$ when $U_{j(k)}^{(1)} \in$ $\partial \Omega$. We assume $\varepsilon \in\left(0, \varepsilon_{0}\right)$, with $0<\varepsilon<\frac{\varepsilon_{1}}{10}$. We introduce locally near $U_{j(k)}^{(1)}$ a new coordinate system $\left(\tilde{x}_{1}, \ldots, \tilde{x}_{n}\right)$ by application of Lemma 3.3.1 with $f_{1}=f+\Phi$ and $\alpha=\left.(f+\Phi)\right|_{\partial \Omega}$.

Hence, we can write in $B\left(U_{j(k)}^{(1)}, 2 \varepsilon_{1}\right)$, choosing $\varepsilon_{1}$ small enough:

$$
(f+\Phi)(\tilde{x})=-\tilde{x}_{n}+\left.(f+\Phi)\right|_{\partial \Omega}\left(\tilde{x}^{\prime}\right)=-\tilde{x}_{n}+f\left(\tilde{x}^{\prime}, 0\right)+\varphi\left(\tilde{x}^{\prime}\right)
$$

with an arbitrary choice of $\tilde{x}^{\prime}$ in the boundary.

Remark moreover that in this case,

$$
d \tilde{x}_{n}\left(U_{j(k)}^{(1)}\right)=-2 \frac{\partial f}{\partial n}\left(U_{j(k)}^{(1)}\right) \vec{n}_{U_{j(k)}^{*}}^{(1)} .
$$

We choose the coordinate system $\tilde{x}^{\prime}$ in the boundary like it was chosen in the boundaryless case (see $[\mathrm{HeSj} 4][\mathrm{HKN}]$ ) according to the geometry of stable and unstable manifolds in order to write $\left.(f+\Phi)\right|_{\partial \Omega}$ as a function of $n-2$ coordinates:

$$
\left.(f+\Phi)\right|_{\partial \Omega}\left(\tilde{x}^{\prime}\right)=f\left(\tilde{x}^{\prime}, 0\right)+\varphi\left(\tilde{x}^{\prime}\right)=\left.(f+\Phi)\right|_{\partial \Omega}\left(\tilde{x}_{2}, \ldots, \tilde{x}_{n-1}\right) .
$$

Definition 5.5.8. For any $k \in 1, \ldots, m_{0}$ we define the cut-off $\chi_{k, \varepsilon}$ by:

- If $U_{j(k)}^{(1)} \in \Omega, \chi_{k, \varepsilon}=\tilde{\chi}_{k, \varepsilon}$.

- If $U_{j(k)}^{(1)} \in \partial \Omega$, we first construct near $\partial \Omega \cap E_{k}$ the cut-off $\chi_{k, \varepsilon}^{\partial \Omega}$ like it was 
constructed in the boundaryless case (see [HKN] pp. 26-29).

Then, choosing a cut-off

$$
\chi_{n}\left(\tilde{x}_{n}\right) \in \mathcal{C}_{0}^{\infty}\left(\mathbb{R}_{-}\right), \chi_{n}=1 \text { on }\left(-\delta_{\varepsilon}, 0\right]
$$

we take for $\chi_{k, \varepsilon}$ :

$$
\chi_{k, \varepsilon}(\tilde{x})=\chi_{n}\left(\tilde{x}_{n}\right) \chi_{k, \varepsilon}^{\partial \Omega}+\left(1-\chi_{n}\left(\tilde{x}_{n}\right)\right) \tilde{\chi}_{k, \varepsilon} .
$$

Note that $\chi_{k, \varepsilon}$, for $\delta_{\varepsilon}$ small enough, satisfies the same properties as $\tilde{\chi}_{k, \varepsilon}$ in Proposition 5.4.2 and we make that choice. Moreover, according to [HKN] p.28, in a neighborhood of $\left\{\tilde{x}_{1}=0\right\} \cap \partial \Omega$, the cut-off $\chi_{k, \varepsilon}$ only depends on $\tilde{x}_{1}: \chi_{k, \varepsilon}=\chi_{k, \varepsilon}\left(\tilde{x}_{1}\right)$.

\section{Proposition 5.5.9.}

There exist $\varepsilon_{0}$ and sequences $\left(c_{k, m}\right)_{m \in \mathbb{N}^{*}}$, such that the $(\varepsilon, h)$-dependent and $h$-dependent quasimodes $\psi_{k}^{(0)}$ and $\psi_{j}^{(1)}\left((k, j) \in\left\{1, \ldots, m_{0}\right\} \times\left\{1, \ldots, m_{1}\right\}\right.$ and $\left.\varepsilon \in\left(0, \varepsilon_{0}\right]\right)$ satisfy:

$$
\begin{aligned}
& \left|\left\langle\psi_{j}^{(1)} \mid d_{f, h}^{(0)} \psi_{k}^{(0)}\right\rangle\right|=0 \quad \text { if } j \neq j(k), \\
& \left|\left\langle\psi_{j(k)}^{(1)} \mid d_{f, h}^{(0)} \psi_{k}^{(0)}\right\rangle\right|=\gamma_{k}(h) \delta_{j(k)}(h) \theta_{j(k)}(h) e^{-\frac{f\left(U_{j(k)}^{(1)}\right)-f\left(U_{k}^{(0)}\right)}{h}}\left(1+h c_{k}^{1}(h)\right)
\end{aligned}
$$

where $\gamma_{k}(h), \delta_{j(k)}(h)$, and $\theta_{j(k)}(h)$ are defined in Definition 1.0.2 and $c_{k}(h) \sim$ $\sum_{\ell=0}^{\infty} c_{k, \ell} h^{\ell}$.

Proof. The first statement for $j \neq j(k)$ is a consequence of our choice of $\varepsilon_{1}>0$ and $\chi_{k, \varepsilon}$ which gives according to Proposition 5.4.2-c) $\operatorname{supp} \psi_{j}^{(1)} \cap$ $\operatorname{supp} \nabla \chi_{k, \varepsilon}=\emptyset$. We conclude with $d_{f, h}^{(0)} \psi_{k}^{(0)}=C_{\varepsilon, h}\left(d^{(0)} \chi_{k, \varepsilon}\right) e^{-f / h}$.

The second case was completely treated in [HKN] when $U_{j(k)}^{(1)} \in \Omega$ and $U_{k}^{(0)} \in \Omega$. Moreover, in the case when $U_{j(k)}^{(1)} \in \Omega$ and $U_{k}^{(0)} \in \partial \Omega$, the proof done in $[\mathrm{HKN}]$ remains valid if we take the convenient $\gamma_{k}(h)$.

Show now the cases when $U_{j(k)}^{(1)} \in \partial \Omega$ and $U_{k}^{(0)} \in \Omega \cup \partial \Omega$ by adapting the proofs done in [HKN] and [HeNi1].

From Proposition 5.5.6, Proposition 5.5.7, and

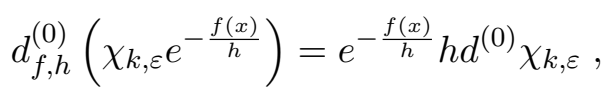

we obtain the existence, for any $\varepsilon>0$, of $\sigma_{\varepsilon}>0$ such that

$$
\begin{aligned}
&\left\langle\psi_{j(k)}^{(1)}\right|\left.d_{f, h}^{(0)} \psi_{k}^{(0)}\right\rangle=h \gamma_{k}(h) \delta_{j(k)}(h) \\
& \times \int_{B\left(U_{j(k)}^{(1)}, \varepsilon\right)}\left\langle b(x, h) \mid d \chi_{k, \varepsilon}\right\rangle(x) e^{-\frac{\left(\Phi(x)+f(x)-f\left(U_{k}^{(0)}\right)\right)}{h}} V_{g_{0}}(d x) \\
& \quad+\mathcal{O}_{\varepsilon}\left(e^{-\frac{f\left(U_{j(k)}^{(1)}\right)-f\left(U_{k}^{(0)}\right)+\sigma_{\varepsilon}}{h}}\right),
\end{aligned}
$$


with $b(x, h)$ defined in Proposition 5.5.7.

Using the coordinate system $\tilde{x}$, with the choice of $\chi_{k, \varepsilon}$,

$$
\begin{aligned}
& \left\langle\psi_{j(k)}^{(1)} \mid d_{f, h}^{(0)} \psi_{k}^{(0)}\right\rangle=h \gamma_{k}(h) \delta_{j(k)}(h) \\
& \times \int_{B\left(\widetilde{\left.U_{j(k)}^{(1)}, \varepsilon\right)}\right.}\left\langle b(\tilde{x}, h) \mid d \chi_{k, \varepsilon}(\tilde{x})\right\rangle(\tilde{x}) e^{-\frac{-\tilde{x}_{n}+\varphi\left(\tilde{x}^{\prime}\right)+f\left(\tilde{x}^{\prime}, 0\right)-f\left(U_{k}^{(0)}\right)}{h}} V_{g_{0}}(d \tilde{x}) \\
& \quad+\mathcal{O}_{\varepsilon}\left(e^{-\frac{f\left(U_{j(k)}^{(1)}\right)-f\left(U_{k}^{(0)}\right)+\sigma_{\varepsilon}}{h}}\right) \\
& =r(h) \int_{\mathcal{C}_{\varepsilon}}\left\langle b(\tilde{x}, h) \mid d \tilde{x}_{1}\right\rangle \chi_{k, \varepsilon}^{\prime}\left(\tilde{x}_{1}\right) e^{\frac{\tilde{x}_{n}-\varphi\left(\tilde{x}^{\prime}\right)-\left(f\left(\tilde{x}^{\prime}, 0\right)-f\left(U_{j(k)}^{(1)}\right)\right)}{h}} V_{g_{0}}(d \tilde{x}) \\
& +\mathcal{O}_{\varepsilon}\left(e^{-\frac{f\left(U_{j(k)}^{(1)}\right)-f\left(U_{k}^{(0)}\right)+\sigma_{\varepsilon}^{\prime}}{h}}\right),
\end{aligned}
$$

where

$$
r(h)=h \gamma_{k}(h) \delta_{j(k)}(h) e^{\frac{f\left(U_{k}^{(0)}\right)-f\left(U_{j(k)}^{(1)}\right)}{h}}
$$

and $\mathcal{C}_{\varepsilon}$ is a cylinder $\left|\tilde{x}^{\prime}\right|<c_{\varepsilon},-c_{\varepsilon}<\tilde{x}_{n}<0$. Expanding $\left\langle b(\tilde{x}, h) \mid d \tilde{x}_{1}\right\rangle$ to a Taylor Series (of arbitrary order), we can obtain, using the Laplace method, an asymptotic expansion (of arbitrary order) for $\left\langle\psi_{j(k)}^{(1)} \mid d_{f, h}^{(0)} \psi_{k}^{(0)}\right\rangle$.

Moreover, the first term in the expansion of $\left\langle b(\tilde{x}, h) \mid d \tilde{x}_{1}\right\rangle$ equals at $\tilde{x}=0$, $\left\langle b_{k, 0}(\tilde{x}) \mid d \tilde{x}_{1}\right\rangle(0)=1$. After recalling (5.5.5) which says that the exponent $f\left(\tilde{x}^{\prime}, 0\right)+\varphi\left(x^{\prime}\right)$ does not depend on $\tilde{x}_{1}$, the first term of the wanted expression is then given by

$$
r(h) \int e^{\frac{\tilde{x}_{n}}{h}} d \tilde{x}_{n} \int e^{-\frac{\varphi\left(\tilde{x}^{\prime}\right)+\left(f\left(\tilde{x}^{\prime}, 0\right)-f\left(U_{j(k)}^{(1)}\right)\right)}{h}} d \tilde{x}_{2} \ldots d \tilde{x}_{n-1} \int \chi_{k, \varepsilon}^{\prime}\left(\tilde{x}_{1}\right) d \tilde{x}_{1} .
$$

Using the Laplace method and

$$
\int_{\mathbb{R}} \chi_{k, \varepsilon}^{\prime}\left(x_{1}\right) d x_{1}=-1,
$$

we find

$$
r(h) \frac{h}{2 \frac{\partial f}{\partial n}\left(U_{j(k)}^{(1)}\right)} \frac{\left|\widehat{\lambda}_{1}^{\partial \Omega}\left(U_{j(k)}^{(1)}\right)\right|^{\frac{1}{2}}}{\left.|\operatorname{det} \operatorname{Hess} f|_{\partial \Omega}\left(U_{j(k)}^{(1)}\right)\right|^{\frac{1}{2}}}(\pi h)^{\frac{n-2}{2}} .
$$




\section{Final proof}

\subsection{Main result}

Recall first some notations.

The generalized critical points with index $0,\left\{U_{k}^{(0)}, k \in\left\{1, \ldots, m_{0}\right\}\right\}$, are labelled according to Subsection 5.3 and the generalized critical points with index 1, $\left\{U_{j(k)}^{(1)}, k \in\left\{2, \ldots, m_{0}\right\}\right\}$, are those introduced in Definition 5.3.3. Moreover, the quantity $\lambda_{k}^{a p p}(\varepsilon, h)$ introduced in Definition 5.4.3 is associated with the quasimodes $\psi_{k}^{(0)}$ and $\psi_{j(k)}^{(1)}$ :

$$
\lambda_{k}^{a p p}(\varepsilon, h)=\left|\left\langle\psi_{j(k)}^{(1)} \mid d_{f, h}^{(0)} \psi_{k}^{(0)}\right\rangle\right|^{2} .
$$

At a generalized critical point $U$ with index $i(i \in\{0,1\})$, the Hessians Hess $f(U)$ or Hess $\left.f\right|_{\partial \Omega}$ are computed in orthonormal coordinates for the metric $g_{0}$, while considering only the tangential coordinates $x^{\prime}=\left(x_{1}, \ldots, x_{n-1}\right)$ for the second case.

At least, for a generalized critical point $U \in W$ with index 1 for $W=\Omega$ or $W=\partial \Omega, \widehat{\lambda}_{1}^{W}(U)$ denotes the negative eigenvalue of Hess $\left.f\right|_{W}(U)$.

With these notations, we have the next theorem, which implies Theorem 1.0.3:

Theorem 6.1.1. Under Assumptions 3.1.1 and 1.0.1, the first eigenvalue $\lambda_{1}(h)$ of $\Delta_{f, h}^{N,(0)}$ is 0 and its $m_{0}-1$ first non zero eigenvalues $\lambda_{2}(h), \ldots, \lambda_{m_{0}}(h)$ admit the following asymptotic expansion. There exist $\varepsilon_{0}>0$ and $\alpha>0$ such that, for any $\varepsilon \in\left(0, \varepsilon_{0}\right]$,

$$
\forall k \in\left\{2, \ldots, m_{0}\right\}, \quad \lambda_{k}(h)=\lambda_{k}^{a p p}(\varepsilon, h)\left(1+\mathcal{O}_{\varepsilon}\left(e^{-\frac{\alpha}{h}}\right)\right) .
$$

Recall also that, from Proposition 5.5.9, for any $\varepsilon \in\left(0, \varepsilon_{0}\right]$,

$$
\lambda_{k}^{a p p}(\varepsilon, h)=\gamma_{k}^{2}(h) \delta_{j(k)}^{2}(h) \theta_{j(k)}^{2}(h) e^{-2 \frac{f\left(U_{j(k)}^{(1)}\right)-f\left(U_{k}^{(0)}\right)}{h}}\left(1+h c_{k}^{1}(h)\right)
$$

where $\gamma_{k}(h), \delta_{j(k)}(h)$, and $\theta_{j(k)}(h)$ are defined in Definition 1.0.2 and $c_{k}^{1}(h)$ admits a complete expansion: $c_{k}^{1}(h) \sim \sum_{m=0}^{\infty} h^{m} c_{k, m}$.

\subsection{Finite dimensional reduction and final proof}

Set first, for $\ell \in\{0,1\}$ :

$$
\forall i \in\left\{1, \ldots, m_{\ell}\right\}, \quad v_{i}^{(\ell)}=1_{\left[0, h^{3 / 2}\right)}\left(\Delta_{f, h}^{N,(\ell)}\right) \psi_{i}^{(\ell)},
$$

where the $\psi_{i}^{(\ell)}$ are the $(\varepsilon, h)$ - and $h$-dependent quasimodes introduced in Definition 5.4.3. 


\section{Remark 6.2.1.}

Note that here again we omit the $(\varepsilon, h)$-dependence (resp. h-dependence) of the functions $v_{k}^{(0)}$ (resp. 1-forms $v_{j}^{(1)}$ ) in the notation.

Recall furthermore the definition of the space $F^{(\ell)}$ given in introduction $(\ell \in\{0,1\})$,

$$
F^{(\ell)}=\operatorname{Ran} 1_{\left[0, h^{\frac{3}{2}}\right)}\left(\Delta_{f, h}^{(\ell)}\right),
$$

which has dimension $m_{\ell}$ according to Theorem 3.1.5.

According to Lemma 2.3.5, Corollary 5.5.4 (for $\ell=0$ ) and Proposition 5.5.5 (for $\ell=1),\left\|1_{\left[h^{3 / 2},+\infty\right)}\left(\Delta_{f, h}^{N,(\ell)}\right) \psi_{i}^{(\ell)}\right\|$ is estimated from above by $\mathcal{O}_{\varepsilon}\left(e^{-\frac{\alpha}{h}}\right)$, which implies the two next propositions:

Proposition 6.2.2. For $\ell \in\{0,1\}$, the $\ell$-forms $\left(v_{i}^{(\ell)}\right)_{i \in\left\{1, \ldots, m_{\ell}\right\}}$ satisfy:

$$
\left\|v_{i}^{(\ell)}-\psi_{i}^{(\ell)}\right\|=\mathcal{O}_{\varepsilon}\left(e^{-\frac{\alpha}{h}}\right)
$$

for some $\alpha>0$ independent of $\varepsilon \in\left(0, \varepsilon_{0}\right]$.

\section{Proposition 6.2.3.}

For $\ell \in\{0,1\}$, the system $\left(v_{i}^{(\ell)}\right)_{i \in\left\{1, \ldots, m_{\ell}\right\}}$ is a basis of $F^{(\ell)}$ satisfying:

$$
V^{(\ell)}:=\left(\left\langle v_{i}^{(\ell)} \mid v_{i^{\prime}}^{(\ell)}\right\rangle\right)_{i, i^{\prime} \in\left\{1, \ldots, m_{\ell}\right\}}=\operatorname{Id}_{\mathbb{C}^{m_{\ell}}}+\mathcal{O}_{\varepsilon}\left(e^{-\frac{\alpha^{\prime}}{h}}\right),
$$

for some $\alpha>0$ independent of $\varepsilon \in\left(0, \varepsilon_{0}\right]$.

Finally, we can also establish:

\section{Proposition 6.2.4.}

There exist $\varepsilon_{0}^{\prime}>0$ and $\alpha^{\prime}>0$ such that, for all $\varepsilon \in\left(0, \varepsilon_{0}^{\prime}\right]$, the estimates

$$
\left|\left\langle v_{j}^{(1)} \mid d_{f, h}^{(0)} v_{k}^{(0)}\right\rangle\right| \leq C_{\varepsilon} e^{-\frac{f\left(U_{j(k)}^{(1)}\right)-f\left(U_{k}^{(0)}\right)+\alpha^{\prime}}{h}}, \quad \text { if } j \neq j(k),
$$

and

$$
\left\langle v_{j(k)}^{(1)} \mid d_{f, h}^{(0)} v_{k}^{(0)}\right\rangle=\left\langle\psi_{j(k)}^{(1)} \mid d_{f, h}^{(0)} \psi_{k}^{(0)}\right\rangle\left(1+\mathcal{O}_{\varepsilon}\left(e^{-\frac{\alpha^{\prime}}{h}}\right)\right),
$$

hold for all $(k, j) \in\left\{1, \ldots, m_{0}\right\} \times\left\{1, \ldots, m_{1}\right\}$.

Proof. Remark first, $1_{\left[0, h^{3 / 2}\right)}\left(\Delta_{f, h}^{N,(1)}\right)$ being a spectral projector and using Corollary 2.3.4:

$$
\begin{aligned}
& \left\langle v_{j}^{(1)} \mid d_{f, h}^{(0)} v_{k}^{(0)}\right\rangle=\left\langle 1_{\left[0, h^{3 / 2}\right)}\left(\Delta_{f, h}^{N,(1)}\right) v_{j}^{(1)} \mid d_{f, h}^{(0)} 1_{\left[0, h^{3 / 2}\right)}\left(\Delta_{f, h}^{N,(0)}\right) \psi_{k}^{(0)}\right\rangle \\
& \quad=\left\langle 1_{\left[0, h^{3 / 2}\right)}\left(\Delta_{f, h}^{N,(1)}\right) v_{j}^{(1)} \mid 1_{\left[0, h^{3 / 2}\right)}\left(\Delta_{f, h}^{N,(1)}\right) d_{f, h}^{(0)} \psi_{k}^{(0)}\right\rangle=\left\langle v_{j}^{(1)} \mid d_{f, h}^{(0)} \psi_{k}^{(0)}\right\rangle .
\end{aligned}
$$


The end of the proof is a straightforward consequence of Proposition 5.5.3, which gives

$$
\left\|d_{f, h}^{(0)} \psi_{k}^{(0)}\right\| \leq C_{\varepsilon} e^{-\frac{f\left(U_{j(k)}^{(1)}\right)-f\left(U_{k}^{(0)}\right)-\alpha^{\prime \prime} \varepsilon}{h}},
$$

Propositions 5.5.9 and 6.2.2.

Proof of Theorem 6.1.1.

By Propositions 6.2 .3 and 6.2.4, the bases $\left(v_{i}^{(\ell)}\right)_{i \in\left\{1, \ldots, m_{\ell}\right\}}$ of $F^{(\ell)}$, for $\ell \in$ $\{0,1\}$, satisfy Assumptions 2.2 and 2.3 of [Lep]. Theorem 2.4 of [Lep] then implies Theorem 6.1.1 (which immediately implies Theorem 1.0.3).

Remark 6.2.5. The conditions of [Lep] are not exactly satisfied here because the one to one map $j$ should act from $\left\{1, \ldots, m_{0}\right\}$ to $\left\{1, \ldots, m_{1}\right\}$, with $\operatorname{dim} F^{(i)}=m_{i}$.

We can easily reduce the study to this last case, by setting:

$$
\bar{m}_{0}=m_{0} \quad, \quad \bar{m}_{1}=m_{1}+1
$$

and,

$$
\overline{F^{(0)}}=F^{(0)} \quad, \quad \overline{F^{(1)}}=F^{(1)} \oplus^{\perp} \mathbb{C} v_{m_{1}+1}^{(1)} .
$$

Setting in addition $j(1)=m_{1}+1$ instead of $j(1)=0$, the conditions of [Lep] are fulfilled.

Note furthermore that the decreasing sequence $\left(\alpha_{k}\right)_{k \in\left\{1, \ldots, \bar{m}_{0}\right\}}$ of [Lep] is then here $\left(f\left(U_{j(k)}^{(1)}\right)-f\left(U_{k}^{(0)}\right)\right)_{k \in\left\{1, \ldots, \bar{m}_{0}\right\}}$ whose first term is by definition $+\infty$.

Acknowledgement: The author would like to thank T. Jecko and F. Nier for profitable discussions.

\section{References}

[Bis] J.M. Bismut. The Witten complex and the degenerate Morse inequalities. J. Differ. Geom. 23, p. 207-240 (1986).

[BEGK] A. Bovier, M. Eckhoff, V. Gayrard, and M. Klein: Metastability in reversible diffusion processes I: Sharp asymptotics for capacities and exit times. JEMS 6(4), p. 399-424 (2004).

[BGK] A. Bovier, V. Gayrard, and M. Klein. Metastability in reversible diffusion processes II: Precise asymptotics for small eigenvalues. JEMS 7(1), p. 69-99 (2004). 
[Bur] D. Burghelea. Lectures on Witten-Helffer-Sjöstrand theory. Gen. Math. 5, p. 85-99 (1997).

[ChLi] Kung Ching Chang and Jiaquan Liu. A cohomology complex for manifolds with boundary. Topological Methods in Non Linear Analysis. Volume 5, p. 325-340 (1995).

[CFKS] H.L Cycon, R.G Froese, W. Kirsch, and B. Simon. Schrödinger operators with application to quantum mechanics and global geometry. Text and Monographs in Physics. Springer Verlag (1987).

[CoPaYc] Y. Colin de Verdière, Y. Pan, and B. Ycart Singular limits of Schrödinger operators and Markov processes. J. Operator Theory 41 (1999), no. 1, 151-173.

[DiSj] M. Dimassi and J. Sjöstrand. Spectral Asymptotics in the semiclassical limit. London Mathematical Society. Lecture Note Series 268. Cambridge University Press (1999).

[Duf] G.F.D. Duff. Differential forms in manifolds with boundary. Ann. of Math. 56, p. 115-127 (1952).

[DuSp] G.F.D. Duff and D.C. Spencer. Harmonic tensors on Riemannian manifolds with boundary. Ann. of Math. 56, p. 128-156 (1952).

[FrWe] M.I. Freidlin and A.D. Wentzell. Random perturbations of $d y$ namical systems. Transl. from the Russian by Joseph Szuecs. 2nd ed. Grundlehren der Mathematischen Wissenschaften. 260. New York (1998).

[GHL] S. Gallot, D. Hulin, and J. Lafontaine. Riemannian Geometry. Universitext, 2nd Edition, Springer Verlag (1993).

[Gil] P.B. Gilkey Invariance theory, the heat equation, and the Atiyah-Singer index theorem.

Mathematics Lecture Series, 11. Publish or Perish, Wilmington (1984).

[Gol] S.I. Goldberg. Curvature and Homology.

Dover books in Mathematics, 3rd edition (1998).

[Gue] P. Guérini. Prescription du spectre du Laplacien de Hodge-de Rham. Annales de l'ENS, Vol. 37 (2), p. 270-303 (2004).

[Hel1] B. Helffer. Etude du Laplacien de Witten associé à une fonction de Morse dégénérée. Publications de l'université de Nantes, Séminaire EDP 1987-88. 
[Hel2] B. Helffer. Introduction to the semi-classical Analysis for the Schrödinger operator and applications. Springer Verlag. Lecture Notes in Mathematics 1336 (1988).

[Hel3] B. Helffer. Semi-classical analysis, Witten Laplacians and statistical mechanics. World Scientific (2002).

[Hen] G. Henniart. Les inégalités de Morse (d'après E. Witten). Seminar Bourbaki, Vol. 1983/84. Astérisque No. 121-122, 43-61, (1985) .

[HKN] B. Helffer, M. Klein, and F. Nier. Quantitative analysis of metastability in reversible diffusion processes via a Witten complex approach. Matematica Contemporanea, 26, pp 41-85 (2004).

[HeNi1] B. Helffer and F. Nier. Quantitative analysis of metastability in reversible diffusion processes via a Witten complex approach: the case with boundary, Matematica Contemporanea Vol.26 p. 41-85, 2004.

[HeSj1] B. Helffer and J. Sjöstrand. Multiple wells in the semi-classical limit I, Comm. Partial Differential Equations 9 (4), p. 337-408, (1984).

[HeSj2] B. Helffer and J. Sjöstrand. Puits multiples en limite semi-classique II -Interaction moléculaire-Symétries-Perturbations. Ann. Inst. H. Poincaré Phys. Théor. 42 (2), p. 127-212 (1985).

[HeSj4] B. Helffer and J. Sjöstrand. Puits multiples en limite semi-classique IV -Etude du complexe de Witten -. Comm. Partial Differential Equations 10 (3), p. 245-340 (1985).

[HeSj5] B. Helffer and J. Sjöstrand. Puits multiples en limite semi-classique V - Etude des minipuits-. Current topics in partial differential equations, p. 133-186, Kinokuniya, Tokyo (1986).

[HKS] R. Holley, S. Kusuoka, and D. Stroock. Asymptotics of the spectral gap with applications to the theory of simulated annealing. J. Funct. Anal. 83 (2), p. 333-347 (1989).

[Kol] V.N. Kolokoltsov. Semi-classical analysis for diffusions and stochastic processes, Lecture Notes in Mathematics 1724. Springer Verlag (2000).

[KoMa] V.N. Kolokoltsov, and K. Makarov. Asymptotic spectral analysis of a small diffusion operator and the life times of the corresponding diffusion process. Russian J. Math. Phys. 4 (3), p. 341-36 (1996).

[Lau] F. Laudenbach. Topologie différentielle. Cours de Majeure de l'Ecole Polytechnique (1993).

[Lep] D. Le Peutrec. Small singular values of an extracted matrix of a Witten complex. To appear in Cubo, A Mathematical Journal. 
[Lep1] D. Le Peutrec. Local WKB construction for boundary Witten Laplacians. IRMAR. Preprint 08-52(2008).

[Mic] L. Miclo. Comportement de spectres d'opérateurs à basse température. Bull. Sci. Math. 119, p. 529-533 (1995).

[Mil1] J.W. Milnor. Morse Theory. Princeton University press (1963).

[Mil2] J.W. Milnor. Lectures on the h-cobordism Theorem. Princeton University press (1965).

[Nie] F. Nier, Quantitative analysis of metastability in reversible diffusion processes via a Witten complex approach, Journées "Equations aux Dérivées Partielles", Exp No VIII, Ecole Polytechnique, 2004.

[Per] A. Persson. Bounds for the discrete part of the spectrum of a semibounded Schrödinger operator. Math. Scandinavica 8, p. 143-153 (1960).

[Sch] G. Schwarz. Hodge decomposition. A method for Solving Boundary Value Problems. Lecture Notes in Mathematics 1607, Springer Verlag (1995).

[Sima] C.G. Simader. Essential self-adjointness of Schrödinger operators bounded from below. Math. Z. 159, p. 47-50 (1978).

[Sim2] B. Simon. Semi-classical analysis of low lying eigenvalues, I. Nondegenerate minima: Asymptotic expansions. Ann. Inst. H. Poincaré Phys. Théor. 38, p. 296-307 (1983).

[Wit] E. Witten. Supersymmetry and Morse inequalities. J. Diff. Geom. 17, p. 661-692 (1982).

[Zha] Weiping Zhang. Lectures on Chern-Weil theory and Witten deformations. Nankai Tracts in Mathematics. Vol. 4. World Scientific (2002). 